Estabilidade do movimento

equatorial de partículas

em magnetosferas planetárias

Maité Kulesza

TESE APRESENTADA

$\mathrm{AO}$

INSTITUTO DE MATEMÁTICA E ESTATÍSTICA

DA

UNIVERSIDADE DE SÃO PAULO

PARA

OBTENÇÃO DO GRAU DE DOUTOR

EM

MATEMÁTICA APLICADA

Área de Concentração: Sistemas Dinâmicos

Orientador: Prof. Dr. Clodoaldo Grotta Ragazzo

- Durante o desenvolvimento deste trabalho, a autora recebeu apoio financeiro do CNPq — São Paulo, 23 de Fevereiro de 2005 - 


\section{Estabilidade do movimento equatorial de partículas em magnetosferas planetárias}

Este exemplar corresponde à redação final da tese devidamente corrigida e defendida por Maité Kulesza e aprovada pela comissão julgadora.

São Paulo, 23 de fevereiro de 2005.

Banca examinadora:

- Prof. Dr. Clodoaldo Grotta Ragazzo (Orientador) - IME-USP

- Prof. Dr. Pedro Antonio Santoro Salomão - IME-USP

- Prof. Dr. Mário Jorge Dias Carneiro - UFMG

- Prof. Dr. Sylvio Ferraz Mello - IAG-USP

- Profa. Dra. Tatiana A. Michtchenko - IAG-USP 
O segredo é não correr atrás das borboletas...

É cuidar do jardim para que elas venham até você.

Mário Quintana 
Às três pessoas que mais desejaram esta tese: Nandinho, Painho e Mainha. 


\section{Agradecimentos}

Esta tese foi escrita não só com muito trabalho mas principalmente com muito "açúcar e afeto". Este é o motivo maior de "Agradecimentos" tão longos...

Ao meu orientador Clodoaldo Grotta Ragazzo por ter me recebido e aceitado como aluna; pelo interessante problema proposto; pela excelente orientação, sempre me estimulando a seguir em frente e; por ter me permitido viver uma vida fora do mundo acadêmico, inclusive me dando a oportunidade de passar um ano fora acompanhando meu marido.

Ao meu amigo e professor José Claudio Vidal não só pelo incentivo mas pelo empenho para que eu recomeçasse em São Paulo.

Ao meu orientador de mestrado Hildeberto Eulálio Cabral por ter começado toda minha história acadêmica.

Ao meu amigo Pedro Antonio Santoro Salomão por toda a ajuda nas disciplinas que fizemos juntos e na minha iniciação com programação numérica, pelas sugestões na versão final da tese e pela amizade solícita.

À banca examinadora, professores Mário Jorge Dias Carneiro, Sylvio Ferraz Mello e Tatiana A. Michtchenko, pelas observações e sugestões na defesa da tese.

Aos meus professores de graduação e mestrado pela minha primeira formação matemática.

Aos professores do MAP e do MAT por toda a atenção e torcida e por me receberem em um ambiente acadêmico cordial. Em especial, aos professores Elói Medina, Sérgio Muniz Oliva, José Antonio Verderesi, Roseli Fernandez, Albert Meads Fisher e Eduardo Colli, por terem me inserido em uma sala de aula onde existe um interesse real para que o aluno aprenda e cresça.

Ao meu marido Nandinho por todo seu amor, carinho, paixão, amizade, companheirismo, força, compreensão e por muitas vezes abdicar dele mesmo para que eu pudesse chegar ao fim da tese.

Aos meus Painho e Mainha por todo amor e carinho mas principalmente por 
representarem tudo de mais sólido que existe em mim.

Aos meus manos, Yuri, Raoni e Uirá, por hoje cuidarem de mim como eu um dia cuidei deles e por serem o maior tesouro que eu possuo.

À minha Tia Irene por ser minha segunda mãe e me acolher no seu aconchegante lar; aos meus Tios Lica e Sérgio, e meus primos Chayenne e Wallace, por estarem presentes em cada momento da minha vida.

À minha família Kulesza, pelos "chás" e almoços onde eu ia buscar energia e alegria para continuar em frente. Em especial ao meu Avô Alexandre(in memorian) que sempre esteve e estará comigo, lá e cá.

Aos meus padrinhos, Lourdinha e Mário, pela torcida mesmo à distância. E a minha "madrinha torta" Sílvia por todas as palavras cheias de luz e vida que eu, desde criança, adoro ouvir.

À minha sogra Nuca pelas orações constantes.

Às minhas cunhadas, Roberta, Amanda e Michelle, pela amizade e carinho.

Aos meus sobrinhos e afilhados, Vlad, Luíza, Marion, Vinícius, Rodrigo e Yasmin por encherem minha vida de ternura infantil, mesmo aqueles que eu ainda não tenha podido conhecer e/ou até batizar.

À minha amiga "gurua" Alice por ter criado um fio condutor em 1998, mesmo que a gente não soubesse, que me trouxe à Sampa em 2000 e por ter andando de braços dados comigo em todo o meu caminho de crescimento.

À minha amiga Fer por toda sua ternura e dedicação comigo, desde o curso de Verão até agora.

Aos meus amigos em Jampa: Alvinho, pela infância maravilhosa que tivemos juntos; Elias, Monik e Josué, Hellena, Stênio, Valdneide e Carlos, pelos tempos de graduação e depois por me fazerem sentir em casa a cada volta à "terrinha" e; Deri e Marcos, Erick, Iragildo, Jefferson, Malu e Galego, Micheline e Antonio, Sinhá e Romeu, pelos momentos de alegria que tivemos em família.

Aos meus amigos em Recife: Aninha, Celinho e Ana Tereza, Claudinha, Katinha, Kátia Evangelista, Leleka, Martinha, Mirele e Tony, Stela, Tâninha, Tekinha, Vivi, 
por terem vivido comigo meus melhores e piores momentos acadêmicos mas também terem compartilhado comigo momentos dos quais sinto imensas saudades. À nossa inocência de querer humanizar o DMAT.

Aos meus amigos que reencontrei em Sampa: Cidoca, Rita e Sales, pelos nossos encontros fervilhantes, cheios de muita regionalidade.

Aos meus amigos em Sampa: Aldo, pela sua amizade sincera e companheira; Viviana e Hector, por compartilharem conosco a "vizinhança" e nos fazer sentir como se estivéssemos com "irmãos" por perto; Fabinho, Jair, Olga, Rudimar, Said e Santos, por me salvarem inúmeras vezes nas "ET's", tendo uma infinita paciência com esta "analfabeta em computação" e também pela amizade e carinho todos os dias; Ariane, Bárbara e Gilberto, Caputi, Carlos Henrique, Cecilia e Pablo, Claudia e Juan, Diane, Débora e Luis Carlos(Charlie Brown), Delhi, Domingo, Elier, Fernando, Glaucio, Irene e Zé, Leandro, Liane e Daniel, Lu e Suzy, Lucia, Major, Maria do Carmo e Heitor, Mariana, Mário, Michel, Milena, Nelson, Palmira, Paola, Pinho, Raquel, Raul, Ricardinho, Rita e Osnel, Robson, Rodrigo, Ronaldo, Sabrina e Daniel, Salvador, Samuca, Sandrinha e Cristian, Sílvia, Sônia e Washington, Suzana, Tatiane, Valquíria, Verinha, Walter pelos nossos encontros no IME, e fora dele, tão leves e calorosos que eu já morro de saudades; minha mais nova amiga Marcela, pelo exemplo de força e vida que é pra mim e; galera da sala 141: Aldemir, Anderson, Andréia, Caio, Fernanda, Gladys, Hilde, Lourdes, Márcio, Neusa, Patrícia, Wellington por terem aguentado a minha "aperriação" diária.

Aos meus amigos do Cepê: minha "personal teacher" Sílvia, Camila, Paki, Yasuko e "de quebra", o Ferreira, pelo exercício diário da amizade.

Aos meus amigos caminhantes: Elô, Nelci, Sotello, Massa, Monica e Raúl, Nacho pelos "caminhos" que cruzamos e que mudaram minha vida.

Aos meus amigos em Portugal: Alessia, Ana e Bruno, Bel e Gildemarks, Carlos, Edison, Ju e Marcus, Marçal, Marília, Marta, Miriam, Nayara e Dáger pelos nossos encontros regados com vinho português, queijo, pão e algumas vezes um bacalhauzinho.

Às minhas professoras de Origami: Alice, minha "Mestra", por me mostrar que origami é muito mais do que dobrar um papel e, Mari pelo prazer do convívio. 
Aos meus amigos do Origami: Amália, Cáu, Danilo, Déa, Fátima, Sandra, Sol, principalmente ao mestre Fausto, pela força e amizade na reta final.

À todos os funcionários do IME: da segurança, da limpeza, da copa, das secretarias, do setor fincanceiro, pelo "Bom Dia" que me trazia alegria e conforto. Em especial a Dona Lucia, Rose, da secretaria do MAP e Alessandra, Feijão, Francisca, Patrícia, Rose, da CPG pela dedicação em atender a todos os meus pedidos.

Ao CNPq e ao meu marido pelo apoio financeiro.

\section{Amigos}

Tenho amigos que não sabem o quanto são meus amigos.

Não percebem o amor que lhes devoto e a absoluta necessidade que tenho deles.

A amizade é um sentimento mais nobre do que o amor.

Eis que permite que o objeto dela se divida em outros afetos, enquanto o amor tem intrínseco o ciúme, que não admite a rivalidade.

E eu poderia suportar, embora não sem dor, que tivessem morrido todos os meus amores, mas enlouqueceria se morressem todos os meus amigos!

Até mesmo aqueles que não percebem o quanto são meus amigos e o quanto minha vida depende de suas existências ...

$A$ alguns deles não procuro, basta-me saber que eles existem.

Esta mera condição me encoraja a seguir em frente pela vida.

Mas, porque não os procuro com assiduidade, não posso lhes dizer o quanto gosto deles.

Eles não iriam acreditar.

Muitos deles estão lendo esta crônica e não sabem que estão incluídos na sagrada relação de meus amigos.

Mas é delicioso que eu saiba e sinta que os adoro, embora não declare e não os procure. 
$E$ às vezes, quando os procuro, noto que eles não tem noção de como me são necessários.

De como são indispensáveis ao meu equilibrio vital, porque eles fazem parte do mundo que eu, trêmulamente construí, e se tornaram alicerces do meu encanto pela vida.

Se um deles morrer, eu ficarei torto para um lado.

Se todos eles morrerem, eu desabo!

Por isso é que, sem que eles saibam, eu rezo pela vida deles.

E me envergonho, porque essa minha prece é, em síntese, dirigida ao meu bem estar.

Ela é, talvez, fruto do meu egoísmo.

Por vezes, mergulho em pensamentos sobre alguns deles.

Quando viajo e fico diante de lugares maravilhosos, cai-me alguma lágrima por não estarem junto de mim, compartilhando daquele prazer ...

Se alguma coisa me consome e me envelhece é que a roda furiosa da vida não me permite ter sempre ao meu lado, morando comigo, andando comigo, falando comigo, vivendo comigo, todos os meus amigos, e, principalmente os que só desconfiam ou talvez nunca vão saber que são meus amigos!

A gente não faz amigos, reconhece-os."

Vinícius de Moraes 


\section{Resumo}

Sob hipóteses físicas simplificadoras, apresenta-se uma formulação das equações de movimento de uma partícula carregada em uma típica magnetosfera planetária, onde todas as constantes físicas do planeta e da partícula se resumem em dois parâmetros: $L$ e $\Lambda$. A partir de tal formulação, é realizado um estudo exaustivo da dinâmica restrita ao plano equatorial, em função de vários parâmetros do problema. Em seguida é feita uma análise da estabilidade dos equilíbrios no plano equatorial com a respeito a perturbações fora do mesmo.

As regiões de Hill para o problema são obtidas em função dos parâmetros do sistema. A análise completa das regiões de Hill é uma das maiores contribuições desta tese.

No estudo da dinâmica restrita ao plano equatorial encontram-se certos laços homoclínicos. A dinâmica próxima a tais laços é estudada usando uma certa aproximação da aplicação de Poincaré. Através do estudo desta aplicação é possível determinar a estabilidade dos laços homoclínicos. 


\section{Abstract}

A simple physical model for the motion of a charged particle in a typical planetary magnetosphere is studied. The problem is scaled in such a way that the equations of motion depend only on two parameters: $L$ and $\Lambda$. Then the dynamics on the equatorial plane and the stability of the equilibria with respect to out of plane perturbations are studied.

The Hill's regions of the problem are fully studied. This is one of the main contributions of this thesis.

Some saddle-center loops are found on the equatorial plane. In the last parte of the thesis the question of stability of these loops is addressed using Poincaré's first return maps. 


\section{Índice}

$\begin{array}{ll}\text { Introdução } & 5\end{array}$

1 Equações de Movimento $\quad 9$

1.1 Equações de Movimento . . . . . . . . . . . . . . . . . . . . . . 9

1.2 Equações de Movimento no Plano Equatorial e Equilíbrios Relativos . 12

1.3 Classificação dos Equilíbrios Relativos . . . . . . . . . . . . . . . . . . 18

2 Regiões de Hill $\quad 23$

2.1 Diagrama de Bifurcação das Regiões de Hill . . . . . . . . . . . . . . 23

2.2 Comportamento da Função Potencial $U$ na Origem e no Infinito . . . 24

2.3 Equilíbrios do Plano Equatorial . . . . . . . . . . . . . . . . . . . . . 29

2.4 Equilíbrios Fora do Plano Equatorial . . . . . . . . . . . . . . . . 43

3 Dinâmica Próxima às Órbitas Homoclínicas ao Sela-Centro $\quad 67$

3.1 Aplicação de Poincaré e sua Aproximação Discreta . . . . . . . . . 67

3.2 O Invariante $\alpha$ e sua Interpretação Geométrica . . . . . . . . . . . . 70

3.3 Cálculo Aproximado do Invariante $\alpha \ldots \ldots$. . . . . . . . . . 72

3.4 Estabilidade Orbital dos Laços Sela-Centro . . . . . . . . . . . . . . . 74 
3.5 Atratores para a Aplicação Discreta no Toro . . . . . . . . . . . . . 77

A Dados Planetários de Júpiter e Saturno

B Programas numéricos

Referências Bibliográficas

125 


\section{Introdução}

Desde que o sistema dos anéis de Júpiter foi descoberto em 1979 pela nave espacial Voyager 1(Smith et al. (1979), Owen et al. (1979)) revelou-se um crescente interesse pelos anéis ditos etéreos. Tais anéis têm duas características que os tornam tão diferentes dos outros, são elas: a dinâmica de cada uma de suas partículas é mais importante para a sua forma do que os efeitos do conjunto delas, uma vez que a densidade da partícula é baixa, e a maioria das partículas dos anéis tem uma vida limitada(Burns et al. (1984)).

A teoria desenvolvida nesta tese possui aplicações nos estudos de tais anéis etéreos. Aqui, Júpiter será escolhido como modelo uma vez que, ao contrário de Saturno, possui exclusivamente anéis etéreos. Além dos anéis etéreos, como por exemplo o anel F, Saturno possui também anéis com partículas grandes, da ordem de centímetros ou metros, aos quais os resultados aqui são irrelevantes. No entanto, cabe ressaltar que, do ponto de vista da hipótese de que o eixo magnético do planeta está alinhado ao seu eixo de rotação suposta a seguir, Saturno se encaixa melhor ao modelo proposto do que Júpiter.

Os anéis de Júpiter são bem uniformes e têm três componentes: o halo interno, o anel principal e o anel "gossamer" exterior que possui apenas $10^{-6}$ por cento de massa em relação aos outros dois anéis(Burns et al. (1984)). Devido ao curto período de vida das partículas, os anéis têm de ser permanentemente realimentados. Por exemplo, provavelmente a principal fonte de reabastecimento de grãos do anel principal é o satélite Andrastea(Burns et al. (1999)). No apêndice A podem ser encontradas as constantes físicas relacionadas a Júpiter e Saturno, inclusive comparadas com as da 
Terra.

A maioria das partículas nos anéis de Júpiter possuem diâmetro que vai de 0.1 a $100 \mu m$ (Horányi, Cravens (1996)). Devido a um fenômeno eletromagnético explicado em Consolmagno (1983) e Burns et al. (1999) tais partículas adquirem uma carga elétrica e por causa de tal carregamento elas interagem com o campo magnético de Júpiter. Assim, como o da Terra, o campo magnético de Júpiter é aproximadamente o campo de um dipolo, cujo eixo está inclinado com relação ao eixo do planeta em aproximadamente 10 graus(Consolmagno (1983)). Nesta tese, o eixo magnético e o eixo de rotação do planeta serão considerados alinhados, ou seja, a declinação magnética será desprezada. É importante ressaltar que no caso de Saturno a declinação magnética é menor que 1 grau. Ou seja, para Saturno a hipótese de axisimetria é verificada com acurância. Tal hipótese, natural em uma primeira abordagem facilita muito o problema matematicamente. Com isso, o número de graus de liberdade para o movimento da partícula pode ser reduzido de três para dois usando-se a simetria das equações de movimento com relação ao eixo de rotação do planeta. Uma discussão detalhada acerca da validade do modelo físico aqui utilizado pode ser encontrada em Consolmagno (1983), Burns et al. (1984), Schaffer, Burns (1994) e Krivov et al. (2002).

Neste trabalho, os resultados matemáticos obtidos não serão confrontados com os dados observacionais(encontrados, por exemplo, em Ockert-Bell et al. (1999)), a maioria dos quais obtidos pela sonda Galileo, que recentemente visitou o planeta. Tanto um tratamento de declinação magnética quanto uma confrontação dos resultados com os dados observacionais serão objeto de um futuro estudo.

O principal objetivo deste trabalho é o estudo da estabilidade do movimento no plano equatorial das partículas nos anéis de Júpiter e das regiões de Hill da Hamiltoniana do sistema. A princípio o sistema possui muitos parâmetros, que após um devido reescalamento de variáveis são reduzidos a dois: os parâmetros $L$ e $\Lambda$, onde $L$ é o momento angular e $\Lambda$ é o parâmetro que engloba todas as contantes físicas do problema. A dinâmica no plano equatorial é então exaustivamente estudada em função de $(L, \Lambda)$ e da energia da órbita. Este estudo é motivado pelo fato de que a maior parte dos grãos de poeira encontra-se no plano equatorial do planeta(Schaffer, Burns (1994)). A seguir, apresenta-se um pequeno resumo da tese. 
Este trabalho está dividido em três capítulos. No primeiro deles, descreve-se as várias formulações das equações de movimento para, em seguida, escrevê-las em termos dos parâmetros $L$ e $\Lambda$. De fato, muito da física do problema está contida na definição destes parâmetros adimensionais. Aborda-se, então, o problema no plano equatorial, obtendo-se os equilíbrios e a primeira divisão do plano $(L, \Lambda)$ em termos dos tipos de funções potenciais possíveis. Linearizando o sistema de equações em torno de tais equilíbrios, pôde-se classificá-los quanto à estabilidade nas direções $\rho \mathrm{e}$ $z$ e obter mais uma divisão do plano $(L, \Lambda)$.

As regiões de Hill, que são regiões do espaço de configurações acessíveis ao movimento, são obtidas no segundo capítulo. Primeiramente, uma análise cuidadosa teve de ser feita próxima à "origem", que é uma singularidade do potencial gravitacional e magnético. A seguir, obtêm-se tais regiões para o caso em que todos pontos críticos do potencial estão no plano equatorial. Finalmente, obtêm-se as regiões de Hill em uma região do espaço $(L, \Lambda)$ na qual existem equilíbrios fora do plano equatorial. Deve-se mencionar que um estudo parcial de zonas de Hill já foi feito por Howard et al. (1999), Howard et al. (2000) e Dullin et al. (2002). Para alguns parâmetros fisicamente relevantes do problema tais autores exibiram as zonas de Hill. Uma contribuição principal dada nesta tese foi o estudo completo das zonas de Hill do problema para todos os valores dos parâmetros.

O terceiro capítulo refere-se ao estudo da dinâmica próxima a um tipo especial de órbita, o chamado laço sela-centro. Trata-se de uma órbita homoclínica (ou bi-assintótica) a um equilíbrio do tipo sela-centro (ou seja, associado a um par de autovalores reais $\pm \nu$ e um par de autovalores imaginários puros $\pm \omega i$ ). Os laços selacentro contidos no plano equatorial estão sempre associados a equilíbrios do tipo sela-centro cujo autoespaço do tipo sela está também contido no plano equatorial. A parte do tipo centro é transversal ao plano equatorial. O estudo da dinâmica próxima ao sela-centro é feito através de uma certa aproximação a uma conveniente aplicação de Poincaré, definida sobre uma seção de Poincaré transversal ao laço. Tal aplicação possui dois invariantes $\gamma$ e $\alpha$, aos quais está associada a dinâmica do laço. Um deles, o invariante $\gamma$, é facilmente obtido, uma vez que é dado por $\sqrt{|\omega / \nu|}$. $\mathrm{O}$ invariante $\alpha$ está relacionado à linearização do fluxo na órbita homoclínica. Este foi aqui calculado numericamente. Também neste capítulo faz-se uma breve descrição 
de alguns resultados obtidos por Grotta Ragazzo(Grotta Ragazzo (1997c)) acerca da estabilidade orbital do laço sela-centro. Um deles é um critério numérico que permite extrair informação da estabilidade a partir dos valores de $\gamma$ e $\alpha$. Usando-se tal critério e o cálculo numérico de $\alpha$, foi possível encontrar valores de $\Lambda$ e $L$ para os quais o sistema admite tanto laços sela-centro estáveis quanto instáveis. Quando o laço é instável, foi obtido por Addas Zanata e Grotta Ragazzo(Addas Zanata, Grotta Ragazzo (2002)) um resultado ligado à topologia das órbitas que se afastam do laço sela-centro. Tal resultado é brevemente descrito e também é verificado numérica e explicitamente para o problema estudado. 


\section{Equações de Movimento}

Na primeira seção deste capítulo são apresentadas as equações de movimento de uma partícula nos anéis de Júpiter nas suas formas Newtoniana e Hamiltoniana. Após uma transformação canônica de coordenadas e uma reescala do tempo e da posição, tais equações já na forma Hamiltoniana podem ser reescritas de modo a dependerem de apenas dois parâmetros. O estudo de tais equações no plano equatorial é feito na segunda seção, onde também é dado um gráfico de bifurcação dos tipos de funções potenciais no plano equatorial. Na última seção, obtém-se uma classificação dos tipos de equilíbrios relativos possíveis no plano equatorial.

\subsection{Equações de Movimento}

Considera-se uma partícula de massa $m$ com carga $Q$ sob um campo gravitacional de uma esfera de massa $M$ e um campo magnético de um dipolo girante, localizado no centro da esfera. Supõe-se que o eixo do dipolo, cujo momento magnético é $\mu \mathbf{e}_{z}$, está alinhado com a direção da velocidade angular $\Omega$. Desta forma, a equação de movimento da partícula em um sistema inercial é escrita, no sistema de unidades CGS, como(Mendis et al. (1982)):

$$
m \ddot{\mathbf{q}}=\frac{Q}{c} \dot{\mathbf{q}} \times \mathbf{B}(\mathbf{q})-\frac{Q}{c}(\Omega \times \mathbf{q}) \times \mathbf{B}(\mathbf{q})-M m G \frac{\mathbf{q}}{|\mathbf{q}|^{3}},
$$

onde $\mathbf{q}=(x, y, z) \in \mathbb{R}^{3}, c$ é a velocidade da luz, $G$ é a constante da gravitação universal, $\mathbf{B}(\mathbf{q})=\nabla_{\mathbf{q}} \times \mathbf{A}(\mathbf{q})$ e $\mathbf{A}(\mathbf{q})=\frac{\mu \mathbf{e}_{z} \times \mathbf{q}}{|\mathbf{q}|^{3}}$. 
A Lagrangiana do sistema é dada por:

$$
\mathbf{L}(\mathbf{q}, \dot{\mathrm{q}}, t)=\frac{m}{2}|\dot{\mathbf{q}}|^{2}+\frac{Q}{c} \dot{\mathbf{q}} \cdot \mathbf{A}(\mathbf{q})-\frac{Q}{c}(\Omega \times \mathbf{q}) \cdot \mathbf{A}(\mathbf{q})+\frac{M m G}{|\mathbf{q}|}
$$

segue que o momento associado à $\mathbf{L}$ é dado por:

$$
\mathbf{p}=\nabla_{\dot{\mathbf{q}}} \mathbf{L}=m \dot{\mathbf{q}}+\frac{Q}{c} \mathbf{A}(\mathbf{q}) .
$$

Logo, a Hamiltoniana associada ao sistema é escrita como:

$$
\mathbf{H}(\mathbf{p}, \mathbf{q}, t)=\frac{1}{2 m}\left|\mathbf{p}-\frac{Q}{c} \mathbf{A}(\mathbf{q})\right|^{2}+\frac{Q}{c}(\Omega \times \mathbf{q}) \cdot \mathbf{A}(\mathbf{q})-\frac{M m G}{|\mathbf{q}|} .
$$

Introduzindo-se coordenadas cilíndricas

$$
(x, y, z)=(\rho \cos \varphi, \rho \quad \operatorname{sen} \varphi, z)
$$

onde $\rho \in \mathbb{R}_{+}^{*}$ e $\varphi \in[0,2 \pi]$, e assumindo-se que $\mu=\mu \mathbf{e}_{z}$ e $\Omega=\Omega \mathbf{e}_{z}$, obtém-se a seguinte Hamiltoniana:

$$
H=\frac{1}{2 m}\left[p_{\rho}^{2}+p_{z}^{2}+\left(\frac{p_{\varphi}}{\rho}-\frac{Q \mu}{c} \frac{\rho}{\left(\rho^{2}+z^{2}\right)^{3 / 2}}\right)^{2}\right]+\frac{Q \mu \Omega}{c} \frac{\rho^{2}}{\left(\rho^{2}+z^{2}\right)^{3 / 2}}-\frac{M m G}{\left(\rho^{2}+z^{2}\right)^{1 / 2}}
$$

onde

$$
p_{\varphi}=\rho^{2}\left(m \dot{\varphi}+\frac{Q}{c} \frac{\mu}{\left(\rho^{2}+z^{2}\right)^{3 / 2}}\right)
$$

é constante.

Desta forma, têm-se as equações de movimento:

$$
\begin{aligned}
\dot{\rho} & =\frac{p_{\rho}}{m} \\
\dot{\varphi} & =\frac{1}{m \rho}\left(\frac{p_{\varphi}}{\rho}-\frac{Q \mu}{c} \frac{\rho}{\left(\rho^{2}+z^{2}\right)^{3 / 2}}\right) \\
\dot{z} & =\frac{p_{z}}{m} \\
\dot{p}_{\rho} & =\frac{1}{m}\left[\frac{p_{\varphi}^{2}}{\rho}-3 \frac{Q \mu}{c} \frac{p_{\varphi} \rho}{\left(\rho^{2}+z^{2}\right)^{5 / 2}}-\left(\frac{Q \mu}{c}\right)^{2} \frac{\rho\left(z^{2}-2 \rho^{2}\right)}{\left(\rho^{2}+z^{2}\right)^{4}}\right]-\frac{Q \mu \Omega}{c} \frac{\rho\left(2 z^{2}-\rho^{2}\right)}{\left(\rho^{2}+z^{2}\right)^{5 / 2}}-\frac{M m G \rho}{\left(\rho^{2}+z^{2}\right)^{3 / 2}} \\
\dot{p}_{\varphi} & =0 \\
\dot{p}_{z} & =\frac{3}{m}\left[-\frac{Q \mu}{c} \frac{p_{\varphi} z}{\left(\rho^{2}+z^{2}\right)^{5 / 2}}+\left(\frac{Q \mu}{c}\right)^{2} \frac{\rho^{2} z}{\left(\rho^{2}+z^{2}\right)^{4}}\right]+3 \frac{Q \mu \Omega}{c} \frac{\rho^{2} z}{\left(\rho^{2}+z^{2}\right)^{5 / 2}}-\frac{M m G z}{\left(\rho^{2}+z^{2}\right)^{3 / 2}} .
\end{aligned}
$$


Observa-se que $\varphi$ é uma variável cíclica ou ignorável.

Seja o seguinte reescalamento

$$
\bar{t}=\Omega t
$$

$\mathrm{e}$

$$
\overline{\mathbf{q}}=\left(\frac{\Omega m c}{|Q \mu|}\right)^{1 / 3} \mathbf{q}
$$

Nota-se que $\bar{t}$ e $\overline{\mathbf{q}}$ são adimensionais.

As equações de movimento reescaladas são escritas como:

$$
\begin{aligned}
& \bar{\rho}^{\prime}=p_{\bar{\rho}} \\
& \bar{\varphi}^{\prime}=\frac{1}{\bar{\rho}}\left(\frac{L}{\bar{\rho}}-\operatorname{sgn}(Q \mu) \frac{\bar{\rho}}{\left(\bar{\rho}^{2}+\bar{z}^{2}\right)^{3 / 2}}\right) \\
& \bar{z}^{\prime}=p_{\bar{z}} \\
& p_{\bar{\rho}}^{\prime}=\frac{L^{2}}{\bar{\rho}^{3}}-\operatorname{sgn}(Q \mu) \frac{\bar{\rho}\left(2 \bar{z}^{2}-\bar{\rho}^{2}+3 L\right)}{\left(\bar{\rho}^{2}+\bar{z}^{2}\right)^{5 / 2}}-\frac{\bar{\rho}\left(\bar{z}^{2}-2 \bar{\rho}^{2}\right)}{\left(\bar{\rho}^{2}+\bar{z}^{2}\right)^{4}}-\frac{\Lambda \bar{\rho}}{\left(\bar{\rho}^{2}+\bar{z}^{2}\right)^{3 / 2}} \\
& L^{\prime}=0 \\
& p_{\bar{z}}^{\prime}=3 \bar{z}\left(\operatorname{sgn}(Q \mu) \frac{\bar{\rho}^{2}-L}{\left(\bar{\rho}^{2}+\bar{z}^{2}\right)^{5 / 2}}+\frac{\bar{\rho}^{2}}{\left(\bar{\rho}^{2}+\bar{z}^{2}\right)^{4}}\right)-\frac{\Lambda \bar{z}}{\left(\bar{\rho}^{2}+\bar{z}^{2}\right)^{3 / 2}}
\end{aligned}
$$

onde

$$
L=\bar{\rho}^{2}\left(\bar{\varphi}^{\prime}+\operatorname{sgn}(Q \mu) \frac{1}{\left(\bar{\rho}^{2}+\bar{z}^{2}\right)^{3 / 2}}\right)
$$

(constante) e

$$
\Lambda=\frac{M m G c}{|Q \mu| \Omega}
$$

são também adimensionais.

A Hamiltoniana é dada por:

$$
\bar{H}=\frac{1}{2}\left(p_{\bar{\rho}}^{2}+p_{\bar{z}}^{2}\right)+\bar{U}(\bar{\rho}, \bar{z})
$$

onde

$$
\bar{U}(\bar{\rho}, \bar{z})=\frac{1}{2}\left(\frac{L}{\bar{\rho}}-\operatorname{sgn}(Q \mu) \frac{\bar{\rho}}{\left(\bar{\rho}^{2}+\bar{z}^{2}\right)^{3 / 2}}\right)^{2}+\operatorname{sgn}(Q \mu) \frac{\bar{\rho}^{2}}{\left(\bar{\rho}^{2}+\bar{z}^{2}\right)^{3 / 2}}-\frac{\Lambda}{\left(\bar{\rho}^{2}+\bar{z}^{2}\right)^{1 / 2}} .
$$

É importante salientar que, devido ao mecanismo de carregamento elétrico das partículas(Consolmagno (1983)), a razão entre a força magnética e a força gravitacional, que agem em uma partícula dos anéis, cresce inversamente com o tamanho da 
partícula. Ou seja, para partículas grandes o efeito da força magnética é desprezível, o oposto valendo para as partículas muito pequenas. Portanto, como era de se esperar, o parâmetro físico de maior importância para o entendimento da dinâmica de uma partícula é sua razão $Q / m$. Mais ainda, quando a razão carga-massa converge para o infinito, tem-se o problema de Störmer girante e; quando ela tende para zero, tem-se o problema de Kepler. Na nova parametrização, o primeiro caso equivale a $\Lambda \rightarrow 0$ e o segundo, a $\Lambda \rightarrow \infty$.

\subsection{Equações de Movimento no Plano Equatorial e Equilíbrios Relativos}

No plano equatorial, as equações (1.7)(omitindo-se as barras) são dadas por:

$$
\begin{aligned}
& \rho^{\prime}=p_{\rho} \\
& \varphi^{\prime}=\frac{L}{\rho^{2}}-\operatorname{sgn}(Q \mu) \frac{1}{\rho^{3}} \\
& p_{\rho}^{\prime}=\frac{(\operatorname{sgn}(Q \mu)-\Lambda)}{\rho^{2}}+\frac{L^{2}}{\rho^{3}}-\operatorname{sgn}(Q \mu) \frac{3 L}{\rho^{4}}+\frac{2}{\rho^{5}} \\
& L^{\prime}=0
\end{aligned}
$$

com Hamiltoniana associada

$$
H=\frac{p_{\rho}^{2}}{2}+U(\rho)
$$

onde

$$
U(\rho)=\frac{1}{2}\left(\frac{L}{\rho}-\operatorname{sgn}(Q \mu) \frac{1}{\rho^{2}}\right)^{2}+\frac{(\operatorname{sgn}(Q \mu)-\Lambda)}{\rho} .
$$

Os equilíbrios relativos no plano equatorial são os pontos críticos da função potencial $U$ e portanto, são aqueles para os quais $\rho$ satisfaz:

$$
(-\operatorname{sgn}(Q \mu)+\Lambda) \rho^{3}-L^{2} \rho^{2}+3 \operatorname{sgn}(Q \mu) L \rho-2=0 .
$$

A denominação relativo tem origem no fato de que, na verdade, tais equilíbrios são órbitas periódicas no plano $(\rho, \varphi)$. Se, além da condição (1.13), vale que

$$
\rho=\frac{\operatorname{sgn}(Q \mu)}{L},
$$


então os equilíbrios relativos são, de fato, equilíbrios do sistema. A condição (1.14) é satisfeita no conjunto de parâmetros a seguir:

$$
\{(L, \Lambda): \Lambda=\operatorname{sgn}(Q \mu)\}
$$

Para obter o diagrama de bifurcação da função potencial $U$, será feito um estudo dos pontos críticos, dos limites e dos zeros da função potencial.

\section{Pontos Críticos da Função Potencial $U$}

Observa-se que a equação (1.13), dos pontos críticos de $U$, pode ser reescrita como

$$
\beta \tilde{\rho}^{3}=(\tilde{\rho}-\operatorname{sgn}(Q \mu))(\tilde{\rho}-2 \operatorname{sgn}(Q \mu))
$$

onde

$$
\beta=\frac{-\operatorname{sgn}(Q \mu)+\Lambda}{L^{3}}
$$

e

$$
\tilde{\rho}=L \rho
$$

com $L \neq 0$.

Logo, as bifurcações ocorrem para os pares $(\tilde{\rho}, \beta)$ onde a curva $\beta \tilde{\rho}^{3}$ tangencia a curva $\tilde{\rho}^{2}-3 \operatorname{sgn}(Q \mu) \tilde{\rho}+2$, ou seja, para os pares

$$
\begin{aligned}
& \left(\tilde{\rho}_{1}, \beta_{1}\right)=\left(3-\sqrt{3}, \frac{3-2 \sqrt{3}}{18(2-\sqrt{3})}\right), \\
& \left(\tilde{\rho}_{2}, \beta_{2}\right)=\left(3+\sqrt{3}, \frac{3+2 \sqrt{3}}{18(2+\sqrt{3})}\right),
\end{aligned}
$$

se $Q \mu>0$, ou

$$
\begin{aligned}
& \left(\tilde{\rho}_{3}, \beta_{3}\right)=\left(-3-\sqrt{3}, \frac{-3-2 \sqrt{3}}{18(2+\sqrt{3})}\right), \\
& \left(\tilde{\rho}_{4}, \beta_{4}\right)=\left(-3+\sqrt{3}, \frac{-3+2 \sqrt{3}}{18(2-\sqrt{3})}\right),
\end{aligned}
$$

se $Q \mu<0$.

(a) Caso $Q \mu>0$

Desta forma, para $Q \mu>0$ tem-se que a função potencial possui: 
i. nenhum ponto crítico se
A. $L<0, \Lambda \leq 1$ ou
B. $L>0, \Lambda<\beta_{1} L^{3}+1$;

ii. um ponto crítico se
A. $L<0, \Lambda>1$;
B. $L>0, \Lambda>\beta_{2} L^{3}+1$ ou
C. $L>0, \Lambda=\beta_{1} L^{3}+1$;

iii. dois pontos críticos se
A. $L>0, \Lambda=\beta_{2} L^{3}+1$ ou
B. $L>0, \beta_{1} L^{3}+1<\Lambda<1$;

iv. três pontos críticos se

A. $L>0,1<\Lambda<\beta_{2} L^{3}+1$.

No caso $L=0$, pode-se resolver a equação (1.13) explicitamente e conclui-se que a função $U$ não tem pontos críticos, se $\Lambda \leq 1$, ou possui apenas um, se $\Lambda>1$.

(b) Caso $Q \mu<0$

Já para $Q \mu<0$, tem-se que a função potencial possui:

i. nenhum ponto crítico se
A. $L<0, \Lambda<\beta_{4} L^{3}-1$ ou
B. $L>0, \Lambda \leq-1$;

ii. um ponto crítico se
A. $L<0, \Lambda=\beta_{4} L^{3}-1$;
B. $L<0, \Lambda>\beta_{3} L^{3}-1$ ou
C. $L>0, \Lambda>-1$;

iii. dois pontos críticos se
A. $L<0, \beta_{4} L^{3}-1<\Lambda \leq-1$ ou
B. $L<0, \Lambda=\beta_{3} L^{3}-1$;

iv. três pontos críticos se

A. $L<0,-1<\Lambda<\beta_{3} L^{3}-1$. 
No caso $L=0$, novamente pode-se resolver a equação (1.13) explicitamente e conclui-se que a função $U$ não tem pontos críticos, se $\Lambda \leq-1$, ou possui apenas um, se $\Lambda>-1$.

\section{Zeros da Função Potencial $U$}

A equação dos zeros da função $U$ é dada por

$$
(\operatorname{sgn}(Q \mu)-\Lambda) \rho^{3}+\frac{L^{2} \rho^{2}}{2}-\operatorname{sgn}(Q \mu) L \rho+\frac{1}{2}=0
$$

e é equivalente a equação

$$
2 \beta \tilde{\rho}^{3}=(\tilde{\rho}-\operatorname{sgn}(Q \mu))^{2}
$$

onde $\beta$ e $\tilde{\rho}$ são dados como em (1.16) e (1.17), respectivamente.

As bifurcações desta última equação ocorrem para os pares

$$
\begin{gathered}
\left(\tilde{\rho}_{5}, \beta_{5}\right)=(1,0), \\
\left(\tilde{\rho}_{6}, \beta_{6}\right)=\left(3, \frac{2}{27}\right),
\end{gathered}
$$

se $Q \mu>0$, ou

$$
\begin{gathered}
\left(\tilde{\rho}_{7}, \beta_{7}\right)=\left(-3,-\frac{2}{27}\right), \\
\left(\tilde{\rho}_{8}, \beta_{8}\right)=(-1,0),
\end{gathered}
$$

se $Q \mu<0$.

(a) Caso $Q \mu>0$

Logo, para $Q \mu>0$ o número de zeros se distribui da seguinte forma:

i. nenhum zero se
A. $L<0, \Lambda \leq 1$ ou
B. $L>0, \Lambda<1$;

ii. um zero se
A. $L<0, \Lambda>1$;
B. $L>0, \Lambda>\beta_{6} L^{3}+1$ ou 
C. $L>0, \Lambda=1$;

iii. dois zeros se

A. $L>0, \Lambda=\beta_{6} L^{3}+1$;

iv. três zeros se

A. $L>0,1<\Lambda<\beta_{6} L^{3}+1$.

Do mesmo modo pode-se resolver a equação (1.18) para $L=0$ e obter que $U$ não tem zeros, se $\Lambda \leq 1$, ou possui apenas um, se $\Lambda>1$.

(b) Caso $Q \mu<0$

Agora, para $Q \mu>0$ o número de zeros se distribui da seguinte forma:

i. nenhum zero se
A. $L<0, \Lambda<-1$ ou
B. $L>0, \Lambda \leq-1$;

ii. um zero se
A. $L<0, \Lambda>\beta_{7} L^{3}-1$
B. $L<0, \Lambda=-1$ ou
C. $L>0, \Lambda>-1$;

iii. dois zeros se

A. $L<0, \Lambda=\beta_{7} L^{3}-1$;

iv. três zeros se

A. $L<0,-1<\Lambda<\beta_{7} L^{3}-1$.

Novamente a equação (1.18) pode ser resolvida para $L=0$ e obtém-se que $U$ não tem zeros, se $\Lambda \leq-1$, ou possui apenas um, se $\Lambda>-1$.

\section{Limites da Função Potencial $U$}

Resta o estudo dos limites da função potencial $U$. Observando a expressão (1.12), conclui-se que quando $\rho$ converge para zero, então $U(\rho)$ converge para $+\infty$. No entanto, quando $\rho$ converge para $+\infty$, tem-se que

(a) $U(\rho) \rightarrow 0_{+}$se $\Lambda \leq \operatorname{sgn}(Q \mu)$ ou

(b) $U(\rho) \rightarrow 0_{-}$se $\Lambda>\operatorname{sgn}(Q \mu)$. 


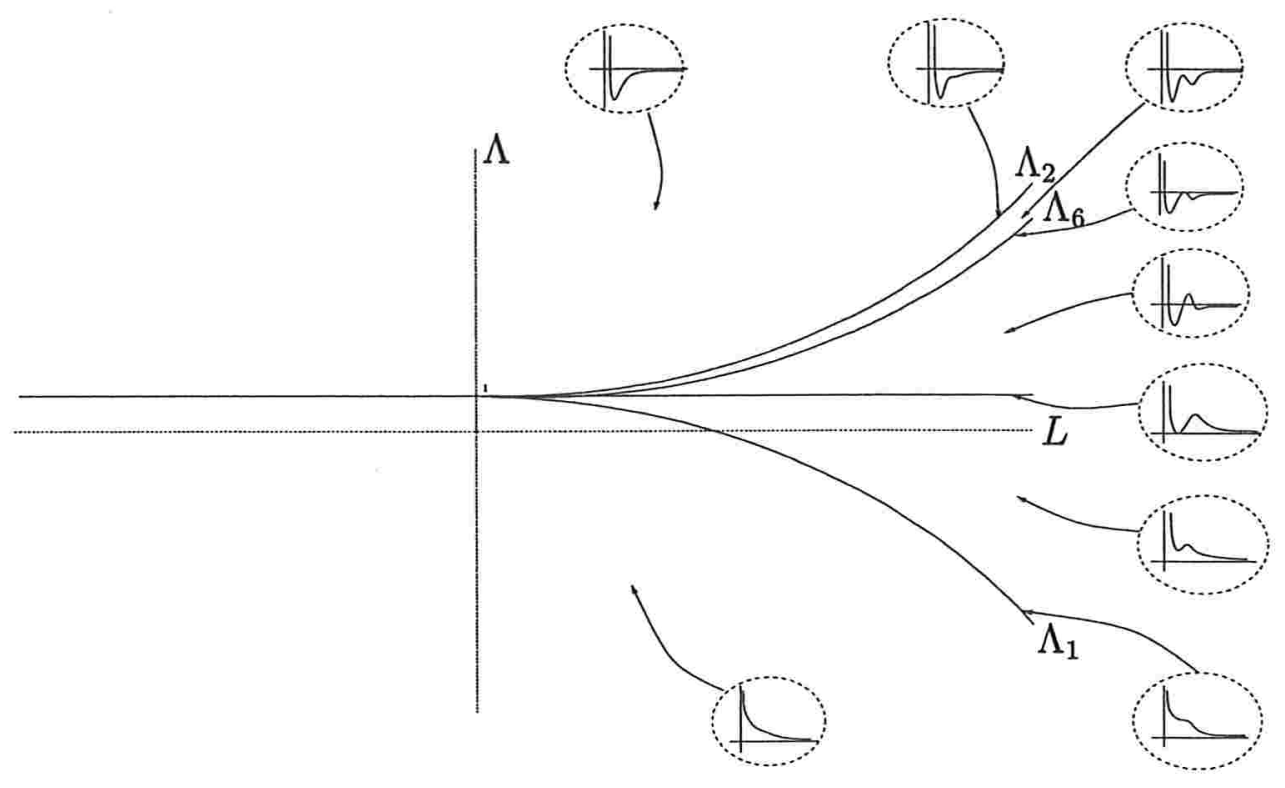

Figura 1.1: Diagrama de bifurcação da função potencial para $Q \mu>0$ onde, com $L \geq 0, \Lambda_{1}(L)=\beta_{1} L^{3}+1, \Lambda_{2}(L)=\beta_{2} L^{3}+1, \Lambda_{6}(L)=\beta_{6} L^{3}+1$.

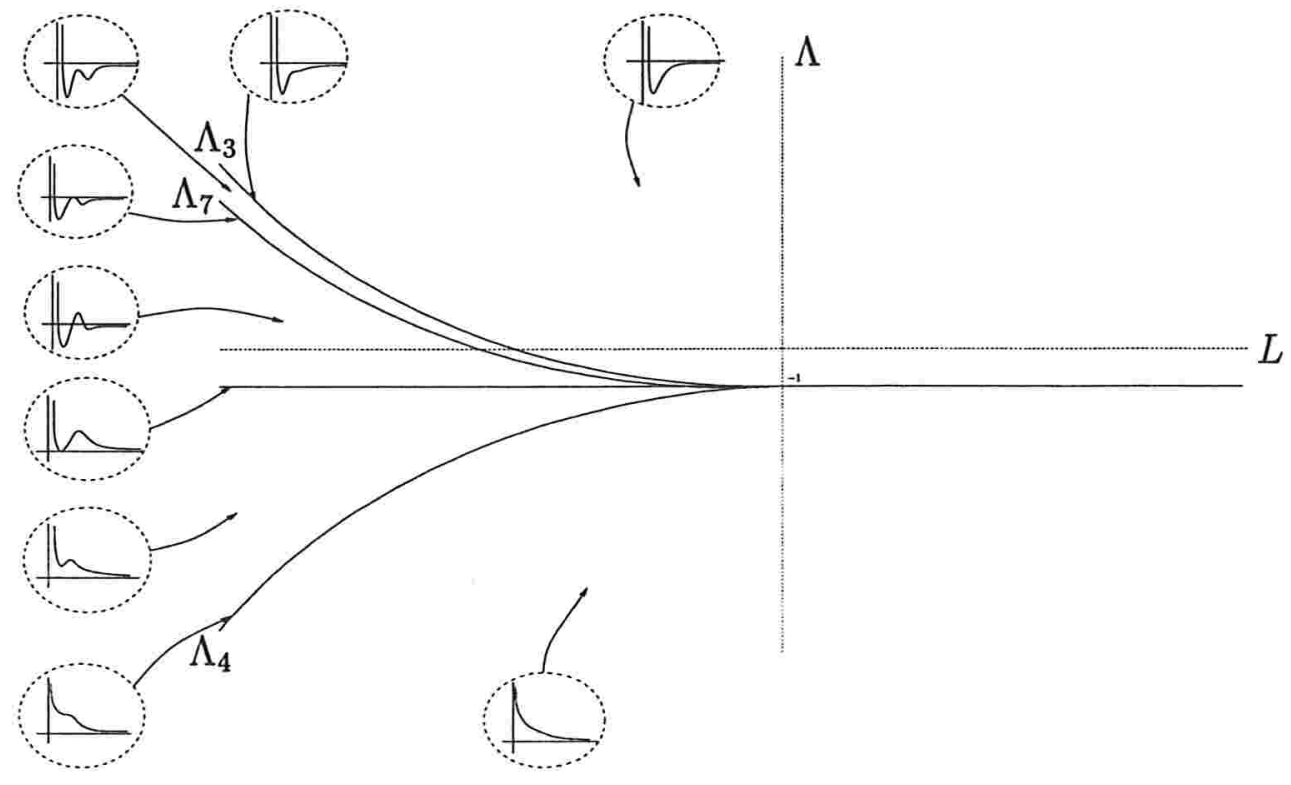

Figura 1.2: Diagrama de bifurcação da função potencial para $Q \mu<0$ onde, com $L \leq 0, \Lambda_{3}(L)=\beta_{3} L^{3}-1, \Lambda_{4}(L)=\beta_{4} L^{3}-1, \Lambda_{7}(L)=\beta_{7} L^{3}-1$. 
Com todos os resultados anteriores, pode-se obter o diagrama de bifurcação da função potencial $U(\rho)$ em termos dos parâmetros $L$ e $\Lambda$ conforme as figuras (1.1) e (1.2) para os casos $Q \mu>0$ e $Q \mu<0$, respectivamente.

\subsection{Classificação dos Equilíbrios Relativos}

Linearizando-se o sistema em torno dos equilíbrios relativos $\rho_{*}$ no plano equatorial, obtém-se que:

$$
\tilde{\zeta}^{\prime}=\tilde{\mathbf{M}} \tilde{\zeta}
$$

onde $\tilde{\zeta}=\left(\rho, \varphi, z, p_{\rho}, L, p_{z}\right)$,

$$
\tilde{\mathbf{M}}=\left(\begin{array}{cccccc}
0 & 0 & 0 & 1 & 0 & 0 \\
H_{L \rho} & 0 & H_{L z} & 0 & H_{L L} & 0 \\
0 & 0 & 0 & 0 & 0 & 1 \\
-H_{\rho \rho} & 0 & -H_{z \rho} & 0 & -H_{L \rho} & 0 \\
0 & 0 & 0 & 0 & 0 & 0 \\
-H_{z \rho} & 0 & -H_{z z} & 0 & -H_{L z} & 0
\end{array}\right)
$$

e $H_{i j}$ são as derivadas segundas do Hamiltoniano $H$ no ponto crítico $\tilde{\zeta}_{*}$, com $i, j=$ $\rho, L, z$.

Restringindo a $(\rho, z)$ e usando que $H_{z \rho}=0$, obtém-se que:

$$
\zeta^{\prime}=\mathrm{M} \zeta
$$

onde $\zeta=\left(\rho, z, p_{\rho}, p_{z}\right)$,

$$
\begin{gathered}
\mathbf{M}=\left(\begin{array}{ccc}
\mathbf{0} & & \mathbf{I} \\
-\nu & 0 & 0 \\
0 & -\omega &
\end{array}\right), \\
\nu=H_{\rho \rho}=\frac{1}{\rho_{*}^{6}}\left(L \rho_{*}-(3 \operatorname{sgn}(Q \mu)+\sqrt{3})\right)\left(L \rho_{*}-(3 \operatorname{sgn}(Q \mu)-\sqrt{3})\right)
\end{gathered}
$$

$\mathrm{e}$

$$
\omega=H_{z z}=\frac{1}{\rho_{*}^{6}}\left((-3 \operatorname{sgn}(Q \mu)+\Lambda) \rho_{*}^{3}+3\left(\operatorname{sgn}(Q \mu) L \rho_{*}-1\right)\right)
$$


O polinômio característico de $\mathbf{M}$ é dado por:

$$
p(\lambda)=\left(\lambda^{2}+\nu\right)\left(\lambda^{2}+\omega\right) .
$$

A matriz (1.23) possui dois subespaços bidimensionais invariantes: um deles tangente ao plano $\left(\rho, p_{\rho}\right)$ e outro tangente ao plano $\left(z, p_{z}\right)$. Os autovalores do subespaço $\left(\rho, p_{\rho}\right)$ estão associados ao coeficiente $\nu$ e os autovalores do subespaço $\left(z, p_{z}\right)$ estão associados ao coeficiente $\omega$.

Nota-se que, usando a equação (1.13), $\omega$ pode ser reescrito como

$$
\omega=\frac{1}{\rho_{*}^{6}}\left(-2 \operatorname{sgn}(Q \mu) \rho_{*}^{3}+L^{2} \rho_{*}^{2}-1\right) .
$$

No entanto, para facilitar a classificação dos equilíbrios, feita a seguir, é usada a expressão (1.25) para $\omega$.

A classificação dos equilíbrios na direção $\rho$, como centro, sela ou parabólico, é imediata da classificação dos pontos críticos do potencial U, como mínimo, máximo ou sela, respectivamente. Para a classificação na direção $z$ é necessário verificar se $o$ autovalor associado a esta direção é real ou complexo o que corresponde a $\omega$ negativo (sela) ou positivo (centro), respectivamente, o que sugere o estudo da equação $\omega=0$. Observa-se que tal equação é equivalente à:

$$
\beta \tilde{\rho}^{3}=3(-\operatorname{sgn}(Q \mu) \tilde{\rho}+1)
$$

onde

$$
\beta=\frac{-3 \operatorname{sgn}(Q \mu)+\Lambda}{L^{3}}
$$

e

$$
\tilde{\rho}=L \rho_{*}
$$

$\operatorname{com} L \neq 0$.

As soluções de (1.28) bifurcam-se para o par:

$$
\left(\tilde{\rho}_{9}, \beta_{9}\right)=\left(\frac{3}{2},-\frac{4}{9}\right)
$$

se $Q \mu>0$, ou

$$
\left(\tilde{\rho}_{10}, \beta_{10}\right)=\left(-\frac{3}{2}, \frac{4}{9}\right)
$$


se $Q \mu<0$.

Zeros da equação $\omega=0$

1. Caso $Q \mu>0$

Neste caso, a equação $\omega=0$ possui

(a) nenhuma solução se
i. $L \leq 0, \Lambda \leq 3$ ou
ii. $L>0, \Lambda<\beta_{9} L^{3}+3$,

(b) uma solução $\left(\rho_{1}\right)$ se
i. $L \leq 0, \Lambda>3$,
ii. $L>0, \Lambda \geq 3$ ou
iii. $L>0, \Lambda=\beta_{9} L^{3}+3$,

(c) duas soluções $\left(\rho_{1}, \rho_{2}\right)$ se

i. $L>0, \beta_{9} L^{3}+3<\Lambda<3$,

No caso (a), tem-se que $\omega<0$ para todo $\rho_{*}$, logo nestas regiões os equilíbrios são todos selas. Por outro lado, no caso (b), têm-se duas situações: em (i) e (ii), $\omega<0$ se $0<\rho_{*}<\rho_{1}, \omega=0$ se $\rho_{*}=\rho_{1}$ e $\omega>0$ se $\rho_{*}>\rho_{1}$; e em (iii), $\omega<0$ se $\rho_{*} \neq \rho_{1}$ e $\omega=0$ se $\rho_{*}=\rho_{1}$. Nestas regiões, a classificação do equilíbrio na direção $z$ depende da sua posição em relação às soluções da equação $\omega=0$, podendo ser do tipo sela, parabólico ou centro em (b(i)) e (b(ii)), e sela ou parabólico em (b(iii)). No caso (c), novamente a classificação depende da posição relativa do equilíbrio, podendo ser sela $\left(\omega<0\right.$ se $0<\rho_{*}<\rho_{1}$ ou $\left.\rho_{*}>\rho_{2}\right)$, parabólico $\left(\omega=0\right.$ se $\rho_{*}=\rho_{1}$ ou $\left.\rho_{*}=\rho_{2}\right)$ ou centro $(\omega>0$ se $\left.\rho_{1}<\rho_{*}<\rho_{2}\right)$. Os casos descritos acima estão sintetizados na figura (1.3).

\section{Caso $Q \mu<0$}

A equação $\omega=0$ neste outro caso possui 


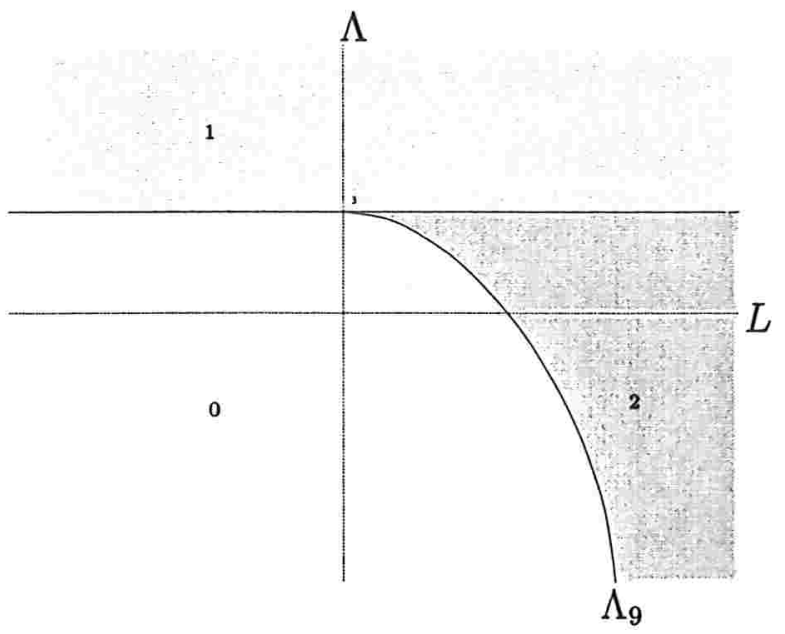

0 nenhum zero(sela);
11 um zero(sela, parabólico ou centro);
[2 dois zeros(sela,parabólico ou centro);
$\Lambda_{9}$ um zero(sela ou parabólico).

Figura 1.3: Classificação do número de soluções da equação $\omega=0$ para $Q \mu>0$ onde, $\operatorname{com} L \geq 0, \Lambda_{9}(L)=\beta_{9} L^{3}+3$.

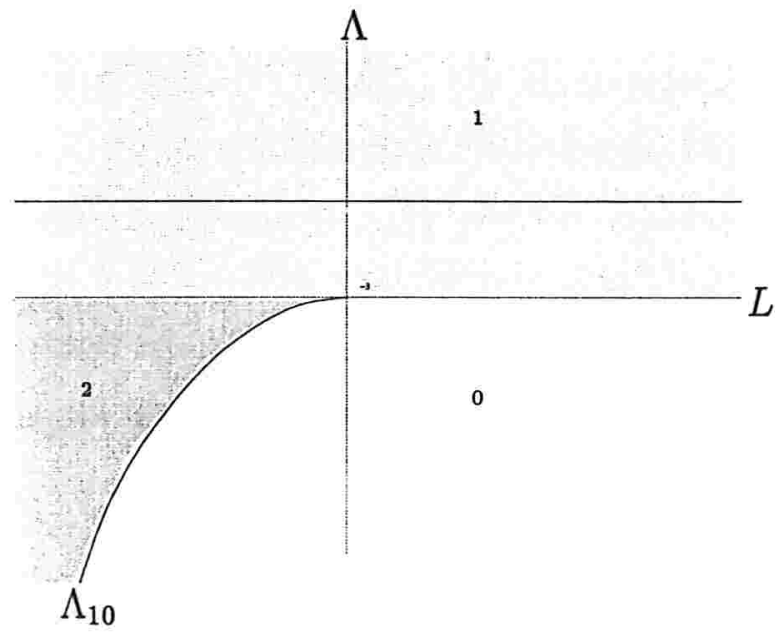

(0) nenhum zero(sela);

[1] um zero(sela, parabólico ou centro);

[2] dois zeros(sela,parabólico ou centro);

$\Lambda_{10}$ um zero(sela ou parabólico).

Figura 1.4: Classificação do número de soluções da equação $\omega=0$ para $Q \mu<0$ onde, $\operatorname{com} L \leq 0, \Lambda_{10}(L)=\beta_{10} L^{3}-3$. 
(a) nenhuma solução se

i. $L<0, \Lambda<\beta_{10} L^{3}-3$ ou

ii. $L \geq 0, \Lambda \leq-3$,

(b) uma solução $\left(\rho_{1}\right)$ se

i. $L<0, \Lambda=\beta_{10} L^{3}-3$,

ii. $L<0, \Lambda \geq-3$ ou

iii. $L \geq 0, \Lambda>-3$,

(c) duas soluções $\left(\rho_{1}, \rho_{2}\right)$ se

i. $L<0, \beta_{10} L^{3}-3<\Lambda<-3$,

Novamente, no caso (a) tem-se que $\omega<0$ para todo $\rho_{*}$, logo nestas regiões os equilíbrios são todos selas. Por outro lado, no caso (b), têm-se duas situações: em (i), $\omega<0$ se $\rho_{*} \neq \rho_{1}$ e $\omega=0$ se $\rho_{*}=\rho_{1}$; e em (ii) e (iii), $\omega<0$ se $0<\rho_{*}<\rho_{1}$, $\omega=0$ se $\rho_{*}=\rho_{1}$ e $\omega>0$ se $\rho_{*}>\rho_{1}$. Nestas regiões, a classificação do equilíbrio na direção $z$ depende da sua posição em relação às soluções da equação $\omega=0$, podendo ser do tipo sela ou parabólico em (b(i)), e sela, parabólico ou centro em (b(ii)) e (b(iii)). No caso (c), novamente a classificação depende da posição relativa do equilíbrio, podendo ser sela $\left(\omega<0\right.$ se $0<\rho_{*}<\rho_{1}$ ou $\left.\rho_{*}>\rho_{2}\right)$, parabólico $\left(\omega=0\right.$ se $\rho_{*}=\rho_{1}$ ou $\left.\rho_{*}=\rho_{2}\right)$ ou centro $\left(\omega>0\right.$ se $\left.\rho_{1}<\rho_{*}<\rho_{2}\right)$. Uma síntese de tais casos pode ser encontrada na figura (1.4). 


\section{Regiões de Hill}

Inicia-se este capítulo com um diagrama de bifurcação dos tipos de regiões de Hill possíveis em termos dos parâmetros $L$ e $\Lambda$, para $Q \mu>0$. E em seguida, explica-se como tais regiões foram obtidas. Em todo o capítulo foi considerado este sinal de $Q \mu$ para fixar idéias. O caso $Q \mu$ negativo é análogo e será apresentado em um trabalho futuro. Na segunda seção, obtém-se o comportamento da função potencial $U$ na origem e no infinito. Com estes resultados e a classificação dos equilíbrios no plano equatorial, pode-se na seção seguinte esboçar as regiões de Hill para o caso onde todos os equilíbrios estão neste plano. Na última seção, mostra-se a existência de equilíbrios fora do plano equatorial e de posse deles, as regiões de Hill para este caso são esboçadas. Todas as regiões esboçadas neste capítulo foram verificadas usando o programa (2) do apêndice B.

\subsection{Diagrama de Bifurcação das Regiões de Hill}

Já foi visto que a Hamiltoniana do problema é dada por:

$$
H(\rho, z)=\frac{1}{2}\left(p_{\rho}^{2}+p_{z}^{2}\right)+U(\rho, z)
$$

onde

$$
U(\rho, z)=\frac{1}{2}\left(\frac{L}{\rho-\operatorname{sgn}(Q \mu)} \frac{\rho}{\left(\rho^{2}+z^{2}\right)^{3 / 2}}\right)^{2}+\operatorname{sgn}(Q \mu) \frac{\rho^{2}}{\left(\rho^{2}+z^{2}\right)^{3 / 2}}-\frac{\Lambda}{\left(\rho^{2}+z^{2}\right)^{1 / 2}} .
$$

Como $\frac{1}{2}\left(p_{\rho}^{2}+p_{z}^{2}\right)$ é não negativo, segue que,

$$
U(\rho, z) \leq E
$$


para cada energia $E$. A região definida pelos pares $(\rho, z)$ que satisfazem a desigualdade anterior é denominada a região de Hill para o nível de energia $E$. Nota-se que o movimento da partícula fica confinado a esta região para a energia fixada $E$.

Fixado o par $(\Lambda, L)$, para cada energia $E$, tem-se uma região de Hill. Para obter a descrição de tais regiões para cada energia, escolhe-se primeiramente uma energia onde tal região seja a mais simples possível. Pelos tipos da função potencial $U$ (ver figura (1.1)) no plano equatorial, o mais conveniente neste caso é a menor energia, ou melhor, o menor valor que o potencial $\left.U\right|_{z=0}$ assume. A seguir, varia-se $E$, neste caso, aumentando a energia, e observa-se a topologia da região $U \leq E$. De fato, este comportamento é alterado a medida que surgem novos pontos críticos e dependendo do tipo dos mesmos. Aqui ressalta-se que importam não somente os equílibrios no plano equatorial, já obtidos no Capítulo 1 , como também os equilíbrios fora do plano equatorial, caso eles existam. De posse de tais equilíbrios, resta apenas obter o comportamento do potencial $U$ na origem (quando $(\rho, z) \rightarrow(0,0)$ ) e no infinito (quando $\rho \rightarrow \infty$ e $z \rightarrow \pm \infty$ ).

O diagrama de bifurcação dos tipos de regiões de Hill possíveis para cada par $(L, \Lambda)$ é dado pela figura (2.1). Neste diagrama estão resumidos os resultados deste capítulo, inclusive com as curvas de bifurcação do número de equilíbrios fora do plano equatorial. Observa-se que o diagrama limita-se a $L \geq 0$. Se $L \leq 0$, tem-se duas situações: uma para $\Lambda \leq 1$, que é idêntica a da região (9) e, outra para $\Lambda \geq 1$, que é esboçada pela figura (2.4).

\subsection{Comportamento da Função Potencial $U$ na Ori- gem e no Infinito}

O potencial $U$ na origem possui três tipos de comportamento que destacam-se a seguir

- Existência de uma região próxima à origem onde $U \leq E$ para toda energia $E$.

- Existência de uma curva equipotencial que se aproxima da origem para $E=L$.

- Próximo da origem, $U>E$ para toda energia $E$. 


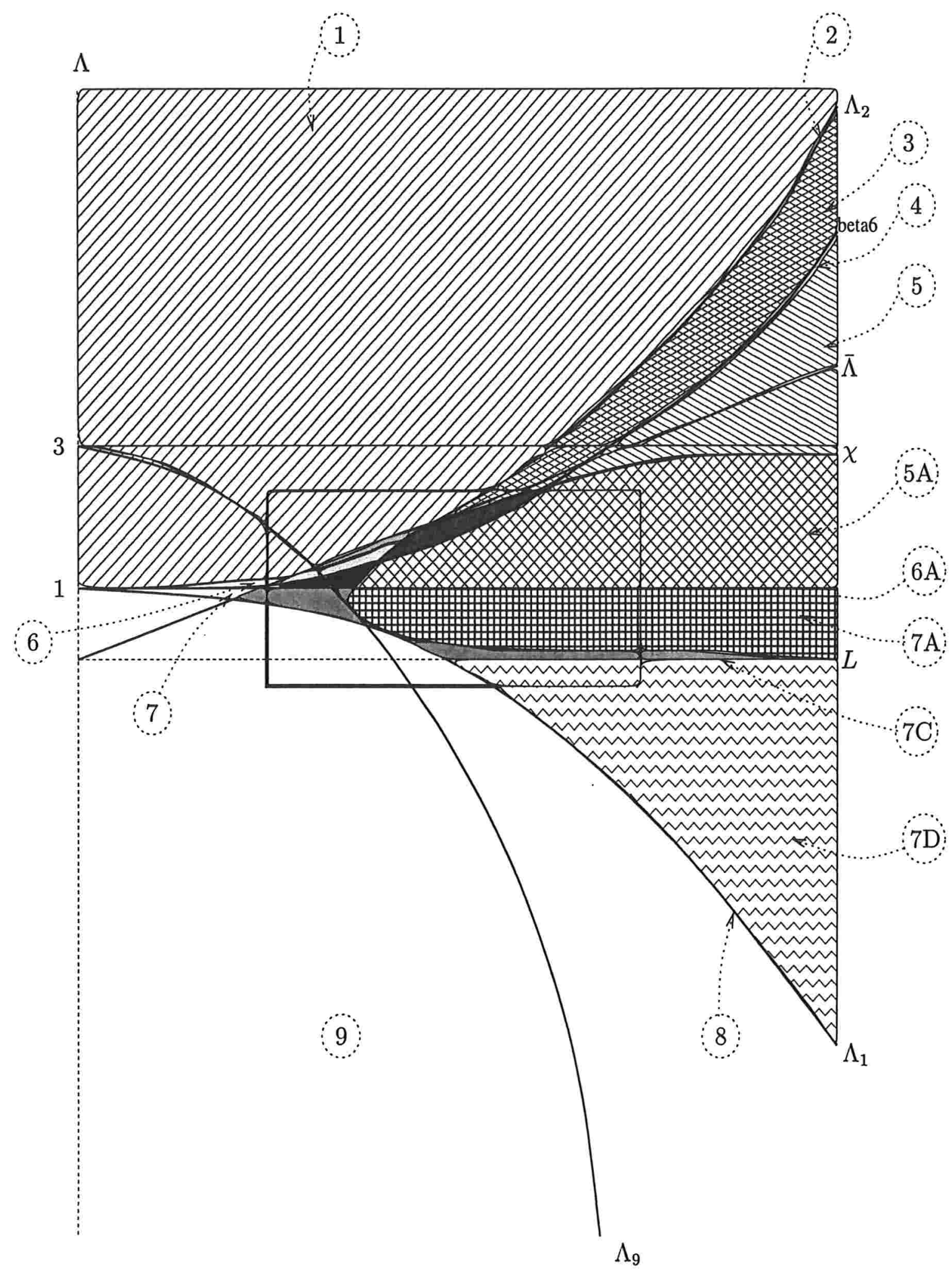

Figura 2.1: Diagrama de bifurcação das regiões de Hill em termos do par $(L, \Lambda)$ para $Q \mu>0$ onde $\Lambda_{1}, \Lambda_{2}, \Lambda_{5}$ são como na figura (1.1), $\Lambda_{9}$ é como na figura (1.3), $\bar{\Lambda}=0,92820323 L$ e $\chi$ obtida numericamente, conforme explicado na seção (2.4). 


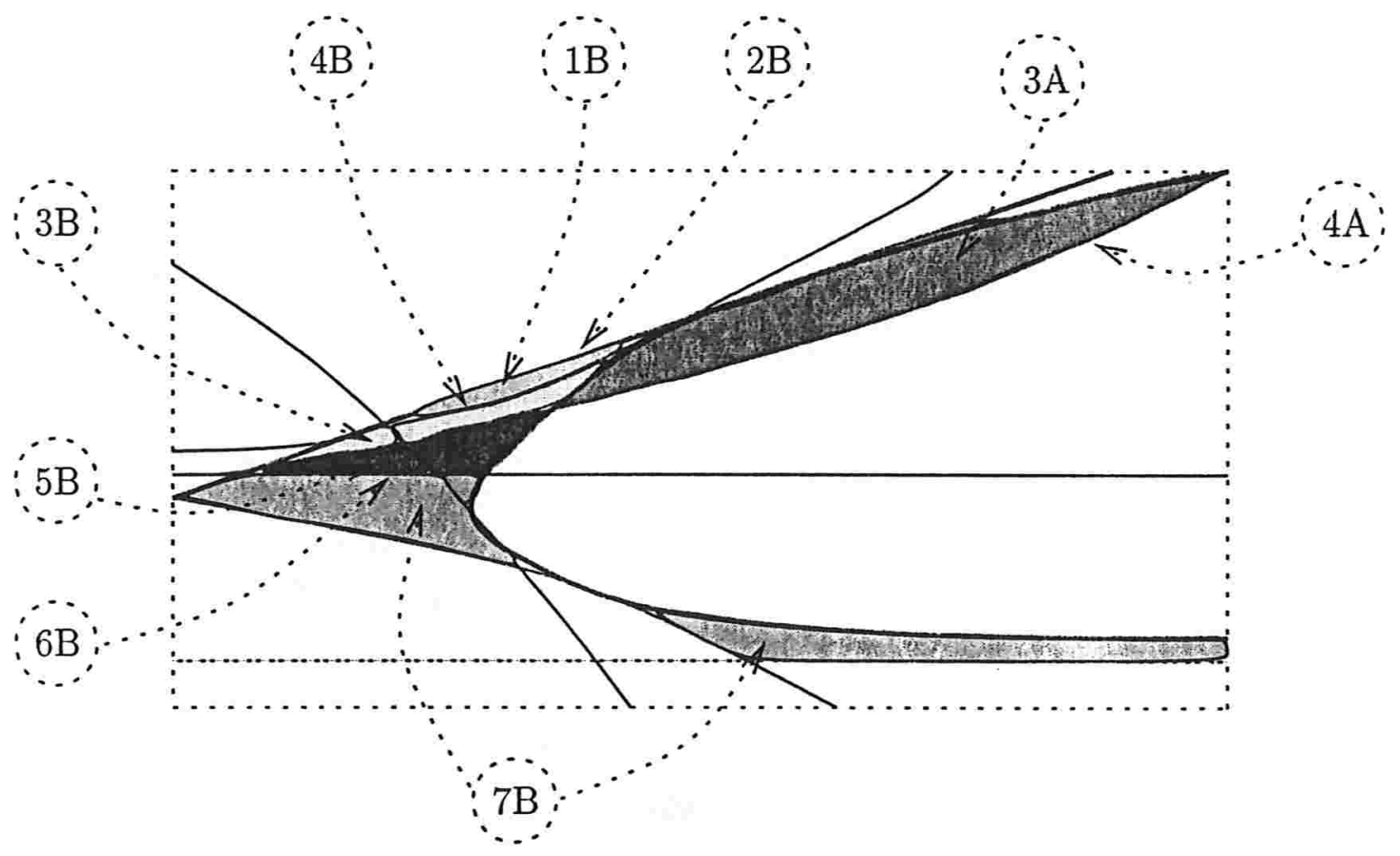

Figura 2.2: "Zoom" da região demarcada na figura (2.1).

Inicialmente, considera-se a mudança de coordenadas dada por

$$
\begin{aligned}
& u=\frac{r^{3}}{\rho^{2}} \\
& r=\sqrt{\left(\rho^{2}+z^{2}\right)}
\end{aligned}
$$

onde $\rho>0$ e $z \in \mathbb{R}$.

Observa-se que, de fato, tem-se um difeomorfismo da região $\{(\rho, z): \rho>0, z>0\}$ na região $\{(r, u): u>0,0<r<u\}$. Analogamente, $\{(\rho, z): \rho>0, z<0\}$ é difeomorfo a $\{(r, u): u>0, r>u>0\}$.

Nestas novas coordenadas, o potencial $U$ é descrito como

$$
U(r, u)=\frac{1}{2 u r^{3}}(L u-\operatorname{sgn}(Q \mu))^{2}+\frac{\operatorname{sgn}(Q \mu)}{u}-\frac{\Lambda}{r}
$$

o qual pode ser reescrito como

$$
U(r, u)=\frac{\operatorname{sgn}(Q \mu)}{u}+\frac{1}{r}\left(-\Lambda+\frac{1}{2 u r^{2}}(L u-\operatorname{sgn}(Q \mu))^{2}\right) .
$$




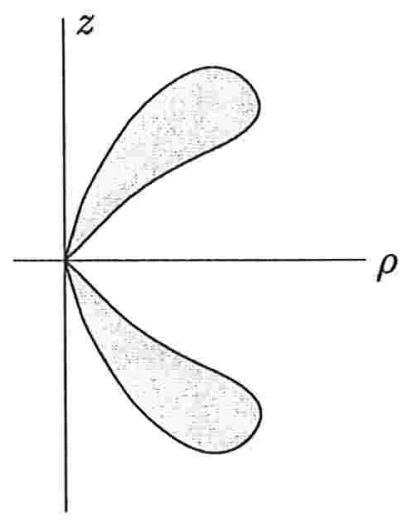

Figura 2.3: Região "salsicha" próxima à origem.

Neste ponto fixa-se o sinal de $Q \mu$ para uma melhor compreensão do comportamento do potencial $U$. Inicialmente, toma-se $Q \mu>0$.

Pode-se reescrever a equação (2.5) como uma equação quadrática em $u$ da seguinte forma

$$
L^{2} u^{2}-2\left(E r^{3}+\Lambda r^{2}+L\right) u+2 r^{3}+1=0
$$

cuja solução é

$$
u=\frac{\left(E r^{3}+\Lambda r^{2}+L\right) \pm r \sqrt{\Delta}}{L^{2}}
$$

onde

$$
\Delta=r^{2}(\Lambda+E r)^{2}+2 L(\Lambda+(E-L) r)
$$

Observa-se que $\Delta \longrightarrow 2 L \Lambda$ e $u \longrightarrow 1 / L$, quando $r \longrightarrow 0$. Desta forma, o estudo do comportamento do potencial $U$ próximo a origem resume-se a analisar os sinais dos limites de $\Delta$ e $u$, ou melhor, do produto $L \Lambda$ e de $L$.

1. Caso $L \Lambda>0$ e $L>0$

Considera-se a equação (2.6). Neste caso, tem-se que $\Delta$ e $u$ convergem para valores positivos, quando $r \rightarrow 0$. Logo, para cada $r$ a equação (2.6) possui dois valores $u$ como solução e portanto, pode-se concluir que existem duas curvas de nível $U=E$ convergindo para zero, o que garante que existe uma região "salsicha" em torno da curva $\bar{\Upsilon}_{1 / L}$ tal que $U(\rho, z)<E$ para toda energia $E$, conforme a figura (2.3). 
Pode-se ver este comportamento, por exemplo, na figura (2.5).

2. Caso $L \Lambda>0$ e $L<0$

Apesar de $\Delta$ convergir pra um valor positivo, quando $r$ converge pra zero, a solução $u$ dada por (2.7) converge para $1 / L$ o qual é um número negativo, o que é uma contradição. Logo, novamente, não existe a região "salsicha".

A figura (2.15) retrata este comportamento.

3. Caso $L \Lambda<0$

Neste caso, pode-se observar que $\Delta$ converge para um valor negativo, quando $r \longrightarrow 0$. Logo, não existe $u$ real que satisfaça a equação (2.6), para $r$ pequeno. E portanto, não pode existir a região "salsicha".

Este comportamento pode ser visto nas figuras (2.4) e (2.15).

4. Caso $L \Lambda=0$

Este caso de divide em dois:

(a) Caso $L=0$

Substituindo $L=0$ na equação (2.6), obtém-se a seguinte equação

$$
-2\left(E r^{3}+\Lambda r^{2}\right) u+2 r^{3}+1=0
$$

e neste caso, quando $r \longrightarrow 0$, a equação acima não possui solução e portanto, a região "salsicha" não aparece.

(b) Caso $\Lambda=0$

Têm-se dua possibilidades:

i. Caso $L>0$

Observa-se que

$$
\Delta=r^{2}(\Lambda+E r)^{2}+2(E-L) r .
$$

Desta forma, se $E \geq L$ então existe a região "salsicha" pois $\Delta \longrightarrow$ $0^{+}$, quando $r$ converge pra zero. Caso contrário, não existe a região "salsicha" pois $\Delta \longrightarrow 0^{-}$, quando $r$ converge pra zero. 
ii. Caso $L<0$

Neste caso, quando $r \longrightarrow 0 u \longrightarrow 1 / L$, o qual é negativo e portanto, não existe a região "salsicha".

O estudo do comportamento de $U$ no infinito é mais simples. Fixando-se $\rho$ na expressão (2.2) de $U$, tem-se que $U$ converge para $\frac{L^{2}}{2 \rho^{2}}$ quando $z \rightarrow \infty$. Por outro lado, se $z$ está fixo, $U$ também converge para 0 , quando $\rho \rightarrow \infty$.

\subsection{Equilíbrios do Plano Equatorial}

Neste momento esboçam-se as regiões de Hill para os casos onde todos os equilíbrios se encontram no plano equatorial. São elas: as regiões (1), (2), (3), (4), (5), (6), (7), (7C), (7D), (8), (9) da figura (2.1).

1. Região onde $L \leq 0$ e $\Lambda>1$ (Figura 2.4)

- Não-existência da região "salsicha", conforme foi visto na seção (2.2).

- O potencial possui apenas um ponto crítico do tipo centro-centro cuja energia é $E_{1}$.

2. Região 1: $L>0$ e $\Lambda>\beta_{2} L^{3}+1$ (Figura 2.5)

- Existência da região "salsicha", de acordo com o caso (1) da seção (2.2).

- O potencial também possui apenas um ponto crítico porém do tipo centrosela e cuja energia é $E_{1}$.

3. Região 2: $L>0$ e $\Lambda=\beta_{2} L^{3}+1$ e $\Lambda>\bar{\beta} L$ (Figura 2.6)

- Existência da região "salsicha", de acordo com o caso (1) da seção (2.2).

- O potencial possui dois pontos críticos: um do tipo centro-sela(energia $E_{1}$ ) e outro do tipo parabólico-sela(energia $E_{3}$ ).

4. Região 3: $L>0$ e $\beta_{2} L^{3}+1<\Lambda<\beta_{6} L^{3}+1$ e $\Lambda>\bar{\beta} L$ (Figura 2.7) 

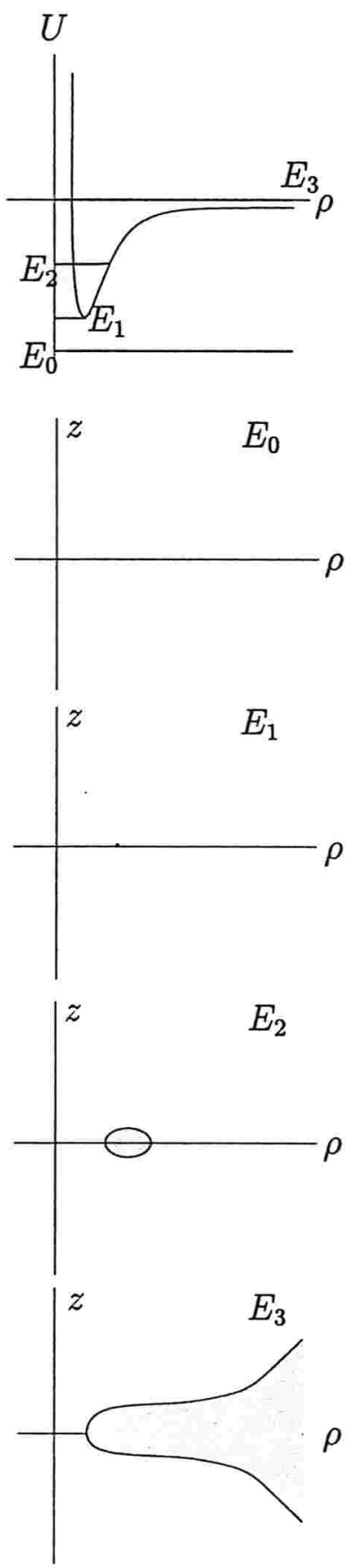

Figura 2.4: Regiões de Hill para o potencial $U$ da região onde $L \leq 0$ e $\Lambda>1$. 

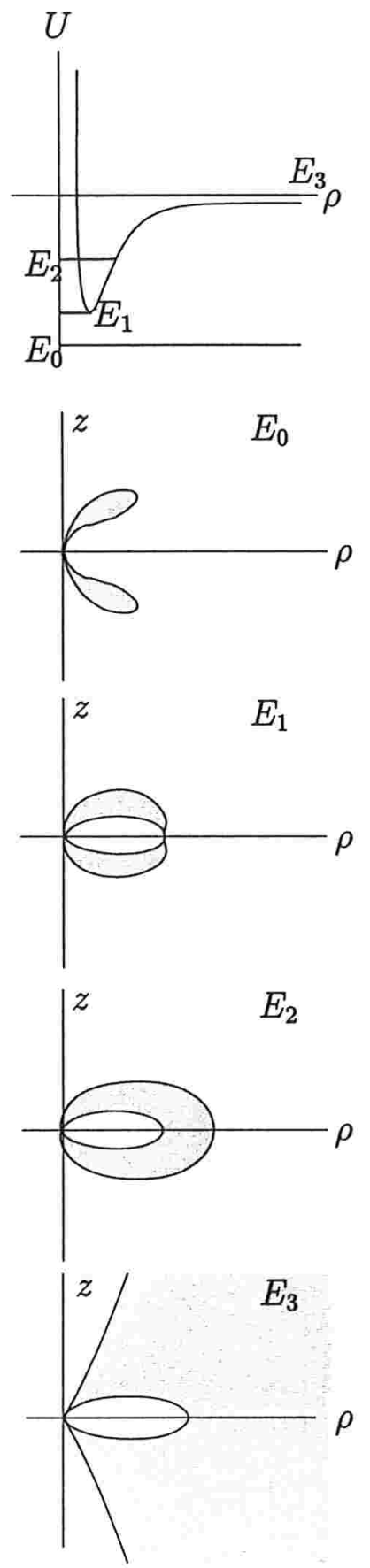

Figura 2.5: Regiões de Hill para o potencial $U$ da região (1) da figura (2.1) acima de curva $\Lambda_{2}$. 

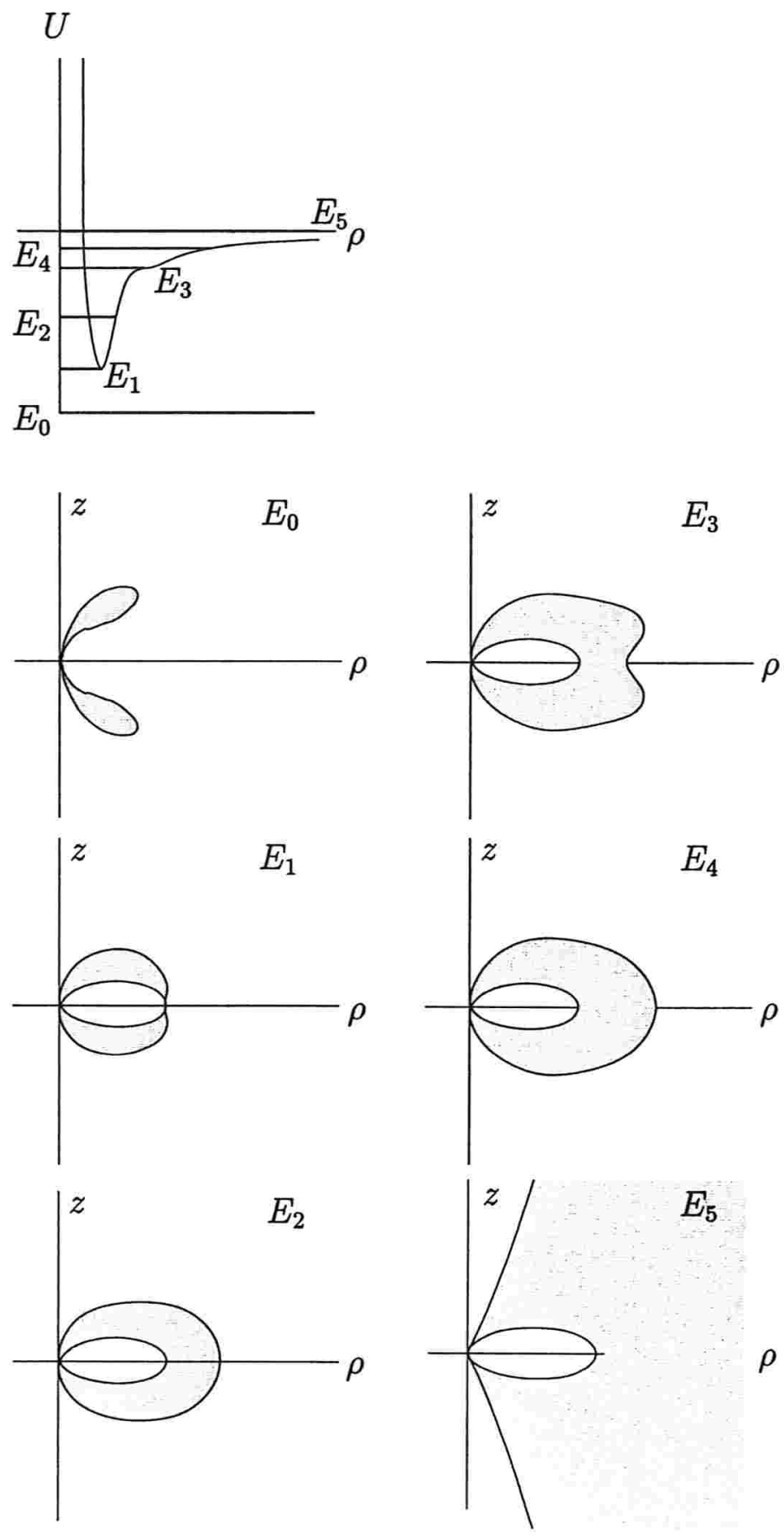

Figura 2.6: Regiões de Hill para o potencial $U$ da região (2) da figura (2.1) sobre a curva $\Lambda_{2}$. 
- Existência da região "salsicha", de acordo com o caso (1) da seção (2.2).

- O potencial possui três pontos críticos: um do tipo centro-sela(energia $E_{1}$ ), outro do tipo sela-centro(energia $E_{5}$ ) e mais um do tipo centrocentro(energia $E_{3}$ ).

5. Região 4: $L>0$ e $\Lambda=\beta_{6} L^{3}+1$ e $\Lambda>\bar{\beta} L$ (Figura 2.8)

- Existência da região "salsicha", de acordo com o caso (1) da seção (2.2).

- O potencial possui três pontos críticos: um do tipo centro-sela(energia $E_{1}$ ), outro do tipo sela-centro(energia $E_{5}$ ) e mais um do tipo centrocentro(energia $E_{3}$ ).

- Este caso difere do anterior pelo fato de que a energia do sela-centro $\left(E_{5}\right)$ coincide com a energia 0 .

6. Região 5: $L>0$ e $\beta_{6} L^{3}+1<\Lambda<1$ e $\Lambda>\bar{\beta} L$ (Figura 2.9)

- Existência da região "salsicha", de acordo com o caso (1) da seção (2.2).

- O potencial possui três pontos críticos: um do tipo centro-sela(energia $E_{1}$ ), outro do tipo sela-centro(energia $E_{6}$ ) e mais um do tipo centrocentro(energia $E_{3}$ ).

- Neste caso, diferentemente dos dois anteriores, a energia do sela-centro $\left(E_{6}\right)$ é maior que a energia $E_{5}=0$.

7. Região 6: $L>0$ e $\Lambda=1$ e $\Lambda>\bar{\beta} L$ (Figura 2.10)

- Existência da região "salsicha", de acordo com o caso (1) da seção (2.2).

- O potencial possui dois pontos críticos: um do tipo centro-sela(energia $\left.E_{1}=0\right)$ e outro do tipo sela-centro(energia $E_{3}$ ).

8. Região 7: $L>0$ e $1<\Lambda<\beta_{1} L^{3}+1$ e $\Lambda>\bar{\beta} L$ (Figura 2.11)

- Existência da região "salsicha", de acordo com o caso (1) da seção (2.2).

- O potencial possui dois pontos críticos: um do tipo centro-sela(energia $E_{1}$ ) e outro do tipo sela-centro(energia $E_{4}$ ). 

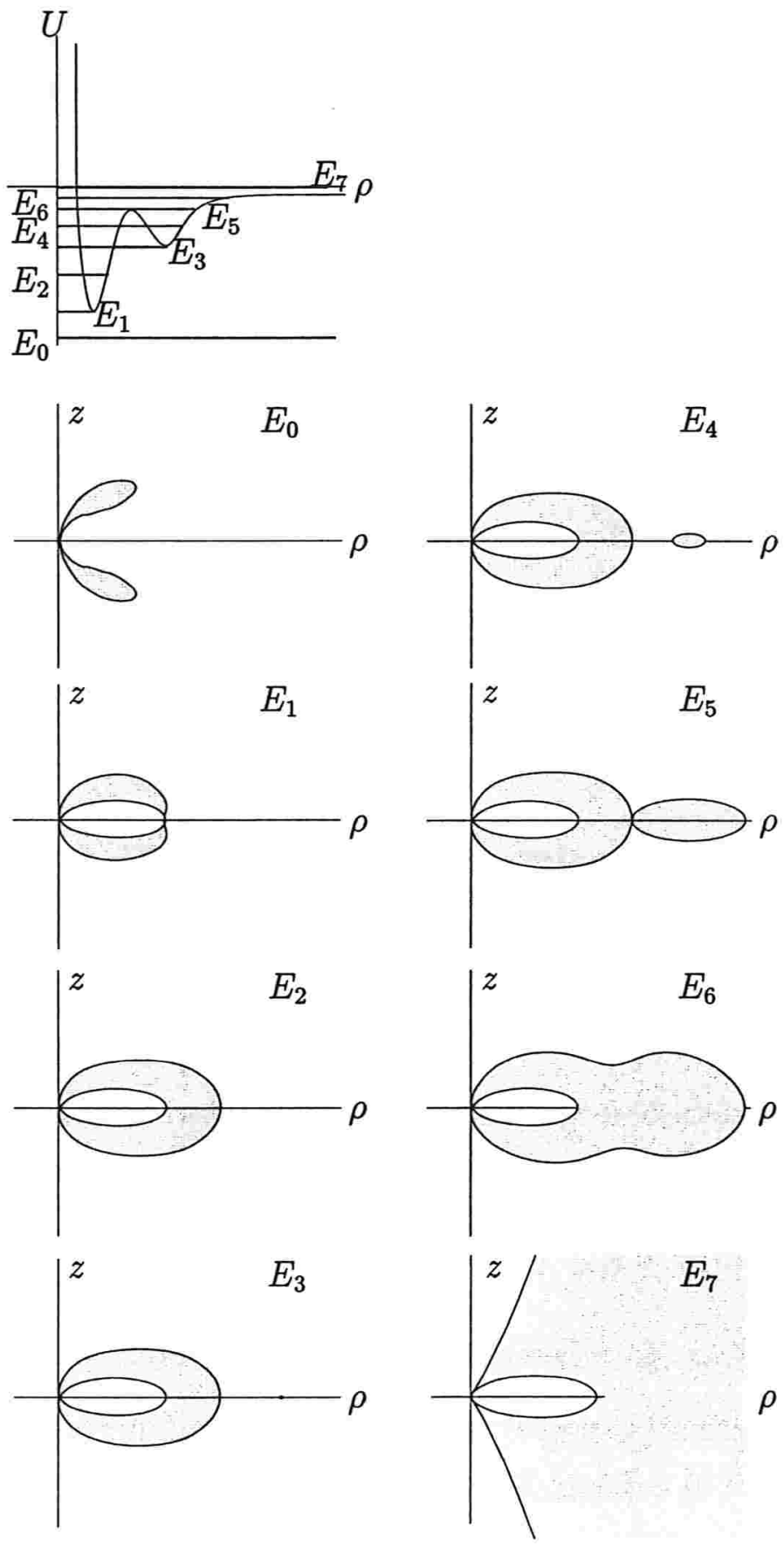

Figura 2.7: Regiões de Hill para o potencial $U$ da região (3) da figura (2.1) entre as curvas $\Lambda_{2}$ e $\Lambda_{6}$. 

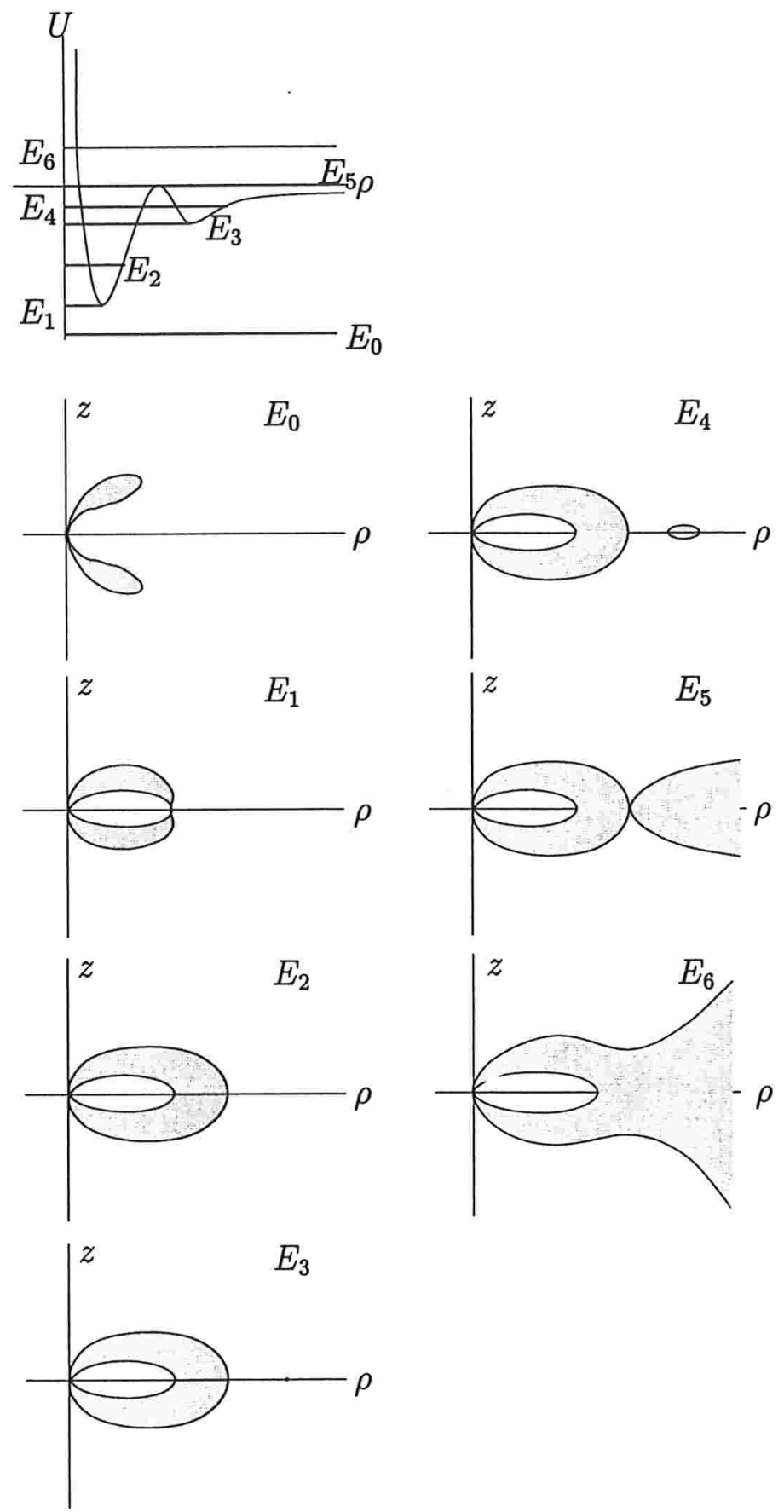

Figura 2.8: Regiões de Hill para o potencial $U$ da região (4) da figura (2.1) sobre a curva $\Lambda_{6}$. 

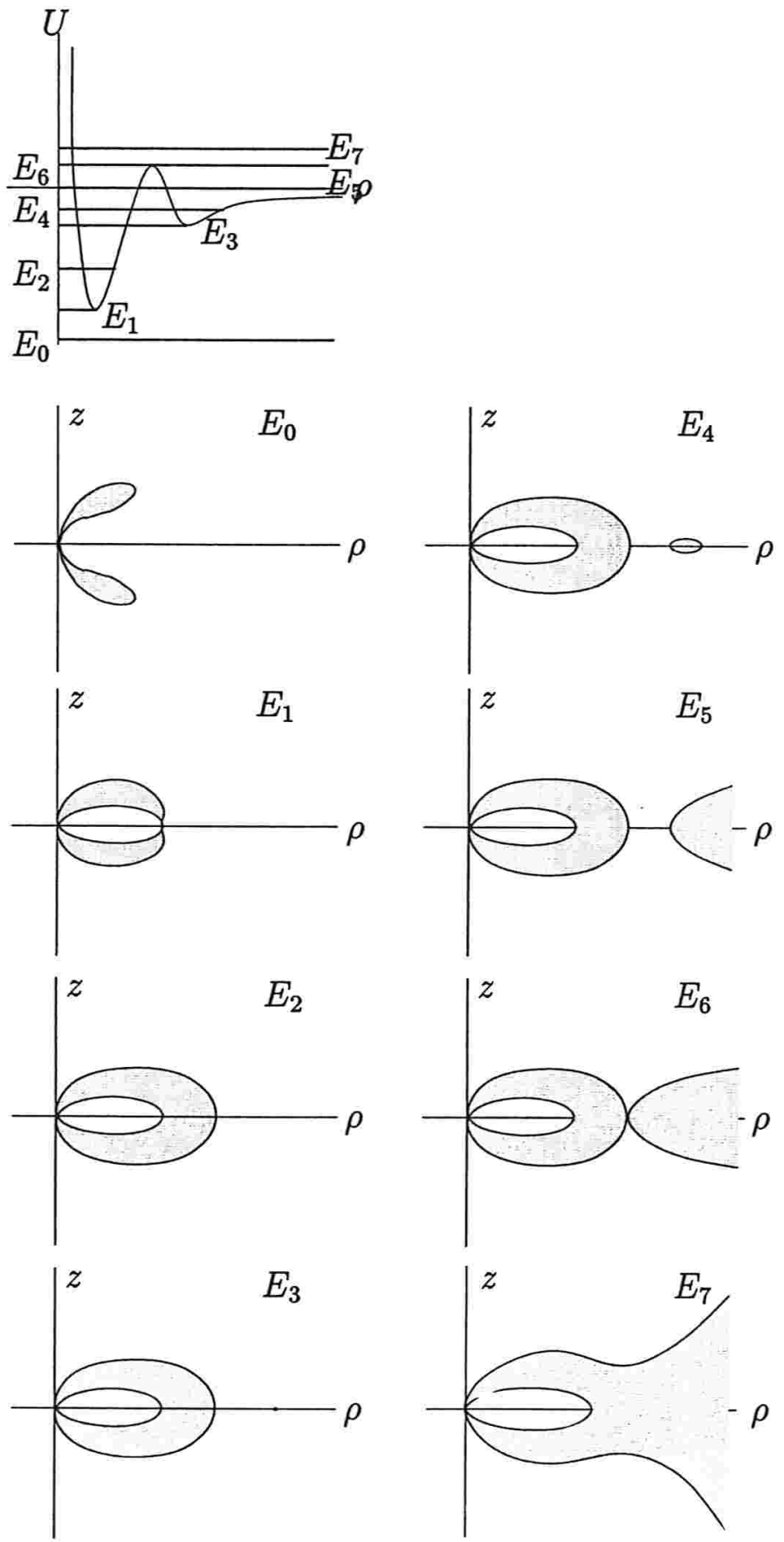

Figura 2.9: Regiões de Hill para o potencial $U$ da região (5) da figura (2.1) entre as curvas $\Lambda_{6}$ e $\Lambda=1$. 

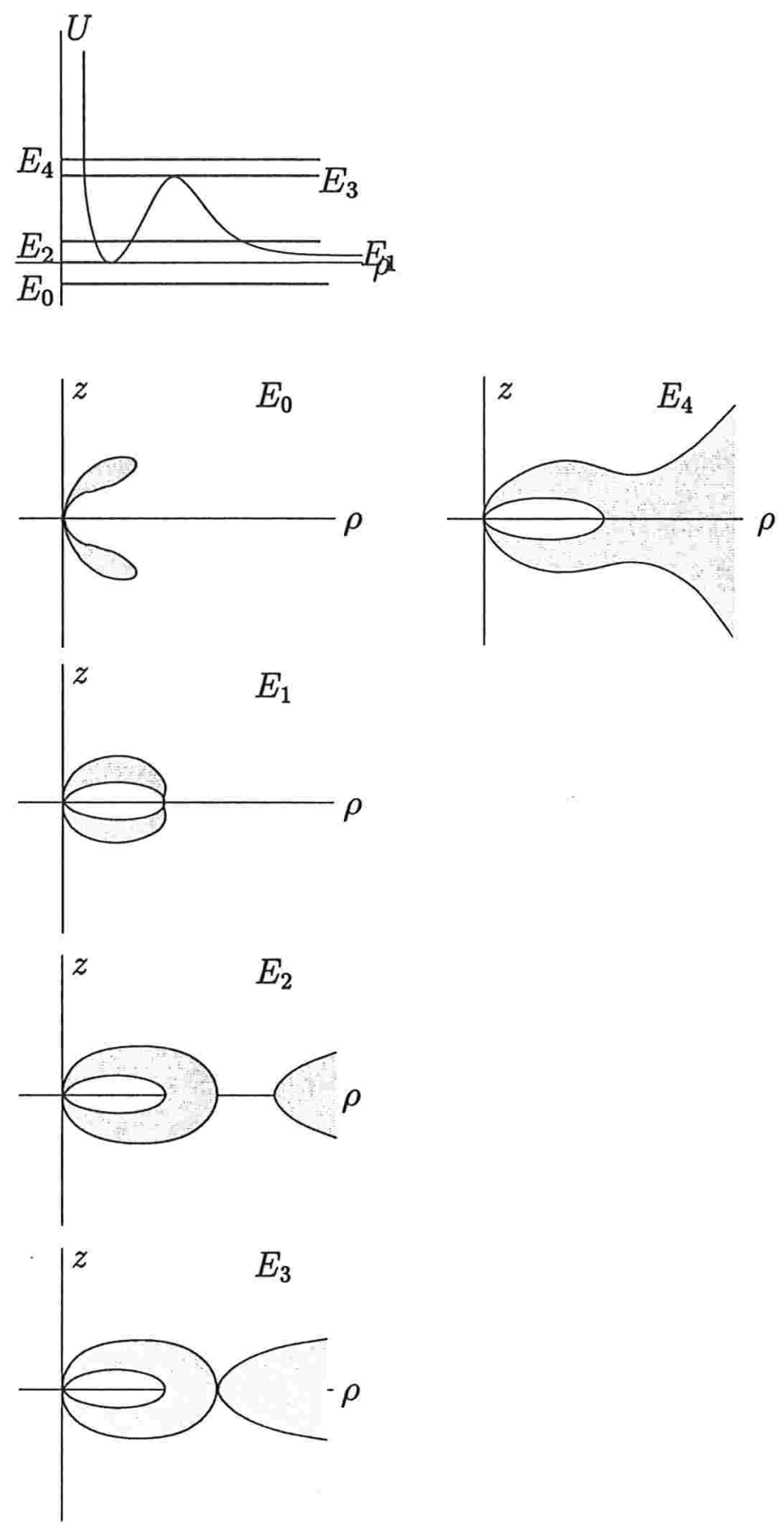

Figura 2.10: Regiões de Hill para o potencial $U$ da região (6) da figura (2.1) sobre a curva $\Lambda=1$. 
- Este caso difere do anterior pelo fato de que a energia do centro-sela $\left(E_{1}\right)$ é maior do que 0 .

9. Região 7C: $L>0$ e $\Lambda=0$ e $\beta_{1} L^{3}+1<\Lambda<\bar{\beta} L$ (Figura 2.12)

- Existência da curva equipotencial quando $E_{4}=L$, conforme foi visto no caso (2) da seção (2.2).

- O potencial possui dois pontos críticos: um do tipo centro-centro(energia $\left.E_{2}\right)$ e outro do tipo sela-centro(energia $E_{6}$ ).

- Neste caso, a região que se origina da "salsicha" existe mas longe da origem. Este é o caso de bifurcação entre o que possui a região "salsicha" $(\Lambda>0)$ e o que não possui $(\Lambda<0)$.

10. Região 7D: $L>0$ e $\beta_{1} L^{3}+1<\Lambda<0$ (Figura 2.13)

- Não-existência da região "salsicha", de acordo com o caso (3) da seção (2.2).

- O potencial possui dois pontos críticos: um do tipo centro-centro(energia $\left.E_{2}\right)$ e outro do tipo sela-centro(energia $E_{4}$ ).

- Neste caso, podemos ver que a região para uma energia $E_{3}>E_{2}$ é um pouco deformada devido ao fato dela estar relacionada com a curva equipotencial(e também com a região "salsicha") do caso anterior.

11. Região 8: $L>0$ e $\Lambda=\beta_{1} L^{3}+1$ (Figura 2.14)

- Não-existência da região "salsicha", de acordo com o caso (3) da seção (2.2).

- O potencial possui apenas um ponto crítico do tipo parabólico-sela(energia $\left.E_{2}\right)$.

12. Região 9: $L>0$ e $\Lambda<\beta_{1} L^{3}+1$ (Figura 2.15)

- Não-existência da região "salsicha", de acordo com o caso (3) da seção (2.2). 

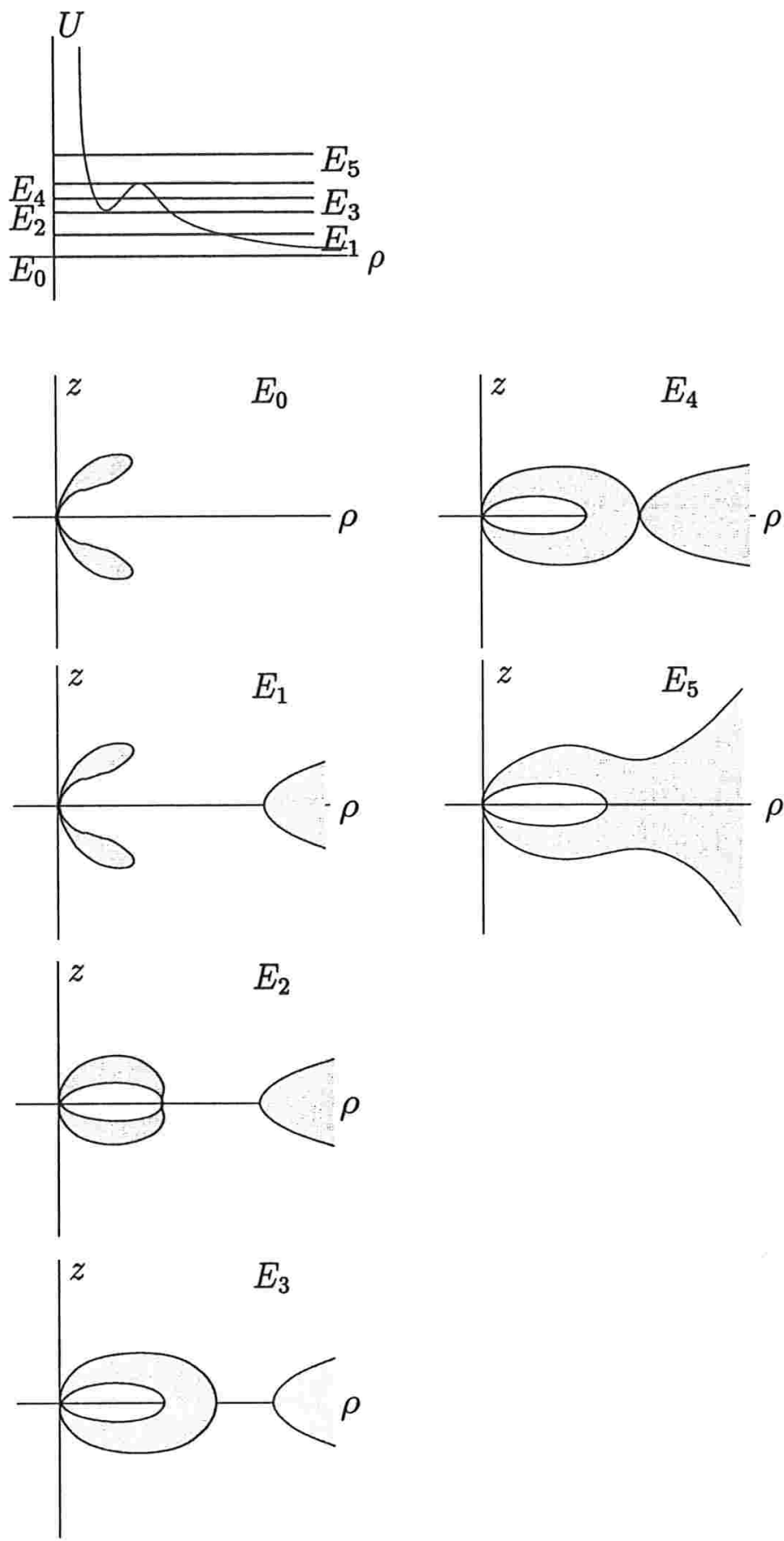

Figura 2.11: Regiões de Hill para o potencial $U$ da região (7) da figura (2.1) entre as curvas $\Lambda=1$ e $\Lambda_{1}$. 

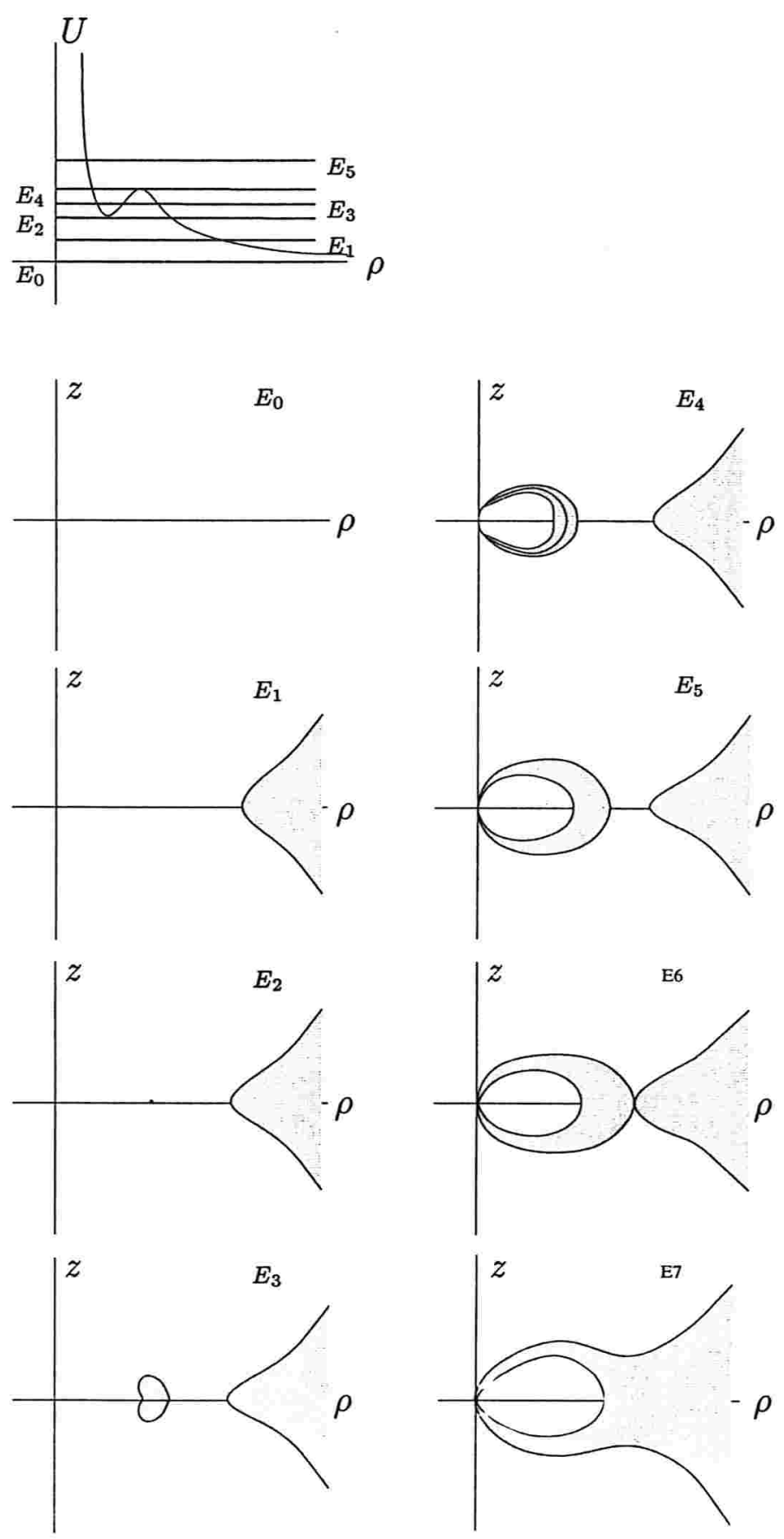

Figura 2.12: Regiões de Hill para o potencial $U$ da região (7C) da figura (2.1) entre as curvas $\Lambda=1$ e $\Lambda_{1}$. 

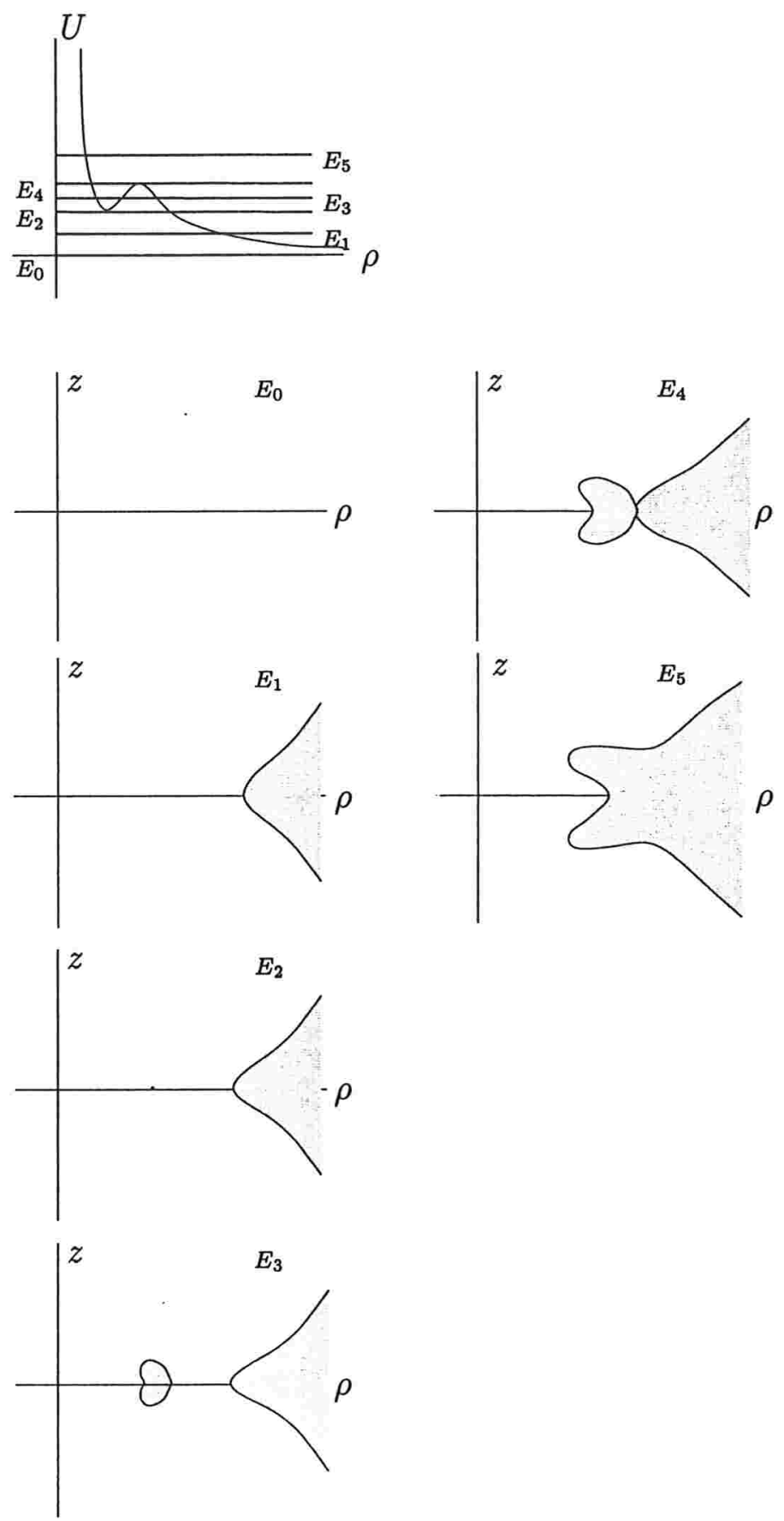

Figura 2.13: Regiões de Hill para o potencial $U$ da região (7D) da figura (2.1) entre as curvas $\Lambda=1$ e $\Lambda_{1}$. 

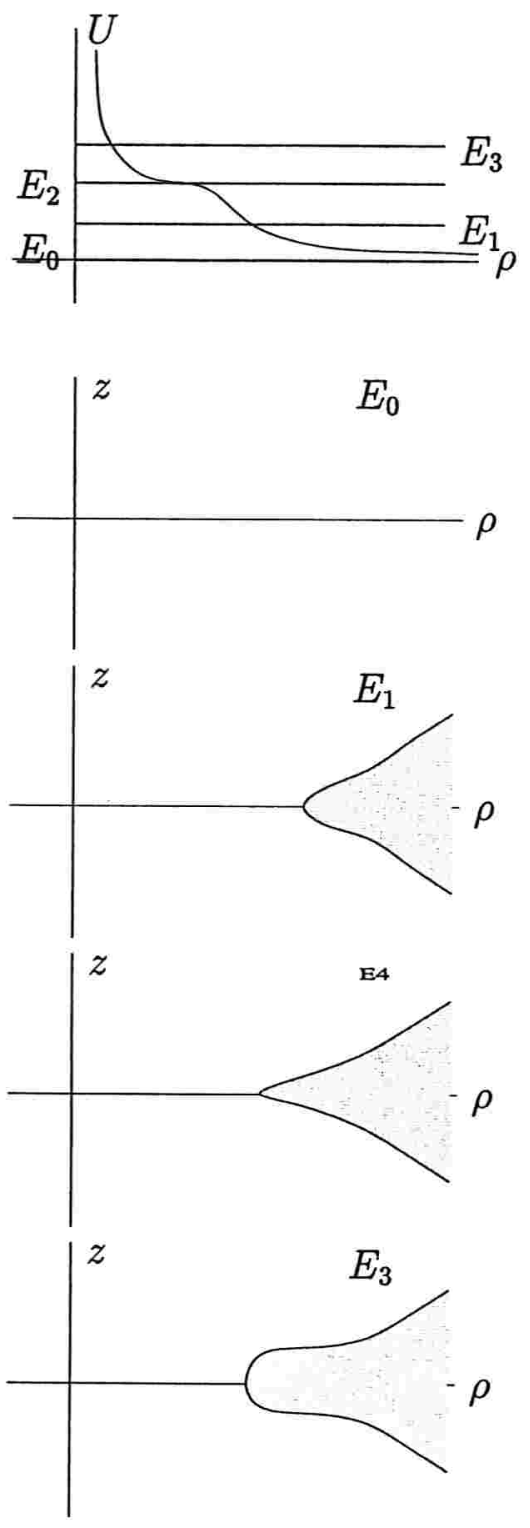

Figura 2.14: Regiões de Hill para o potencial $U$ da região (8) da figura (2.1) sobre a curva $\Lambda_{2}$. 
- O potencial não possui pontos críticos.

13. Região onde $L \leq 0$ e $\Lambda \leq 1$ (Figura 2.15)

- Idêntico à região (9).

\subsection{Equilíbrios Fora do Plano Equatorial}

Para descobrir a existência ou não de pontos críticos fora do plano equatorial, deve-se analisar as equações dos pontos críticos em coordenadas convenientes.

A função potencial pode ser escrita como

$$
U(\rho, r)=\frac{1}{2}\left(\frac{L}{\rho}-\frac{\rho}{r^{3}}\right)^{2}+\frac{\rho^{2}}{r^{3}}-\frac{\Lambda}{r} .
$$

Da expressão (2.10) para a função potencial obtêm-se as derivadas parciais da $U$ :

$$
\begin{aligned}
& \frac{\partial U}{\partial \rho}=-\left(\frac{L}{\rho}-\frac{\rho}{r^{3}}\right)\left(\frac{L}{\rho^{2}}+\frac{1}{r^{3}}\right)+\frac{2 \rho}{r^{3}} \\
& \frac{\partial U}{\partial r}=\frac{1}{r^{2}}\left[\frac{3 \rho}{r^{2}}\left(\frac{L}{\rho}-\frac{\rho}{r^{3}}\right)-\frac{3 \rho^{2}}{r^{2}}+\Lambda\right]
\end{aligned}
$$

Após a substituição de $\tilde{u}=\frac{\rho^{2}}{r^{3}}$ e simplificações, obtém-se que as equações dos pontos críticos são dadas por:

$$
\begin{gathered}
u^{2}\left(1+2 r^{3}\right)-L^{2}=0 \\
3\left(L-u\left(1+r^{3}\right)\right)+\Lambda r^{2}=0
\end{gathered}
$$

as quais são equivalentes a

$$
\begin{gathered}
u=\frac{3 L+\Lambda r^{2}}{3\left(1+r^{3}\right)} \\
2 \Lambda^{2} r^{5}-9 L^{2} r^{4}+12 L \Lambda r^{3}+\Lambda^{2} r^{2}+6 L \Lambda=0
\end{gathered}
$$

Os zeros das últimas equações são os possíveis candidatos a pontos críticos do potencial $U$. No caso $L=0$, é fácil verificar a não-existência de pontos críticos fora do plano equatorial. 

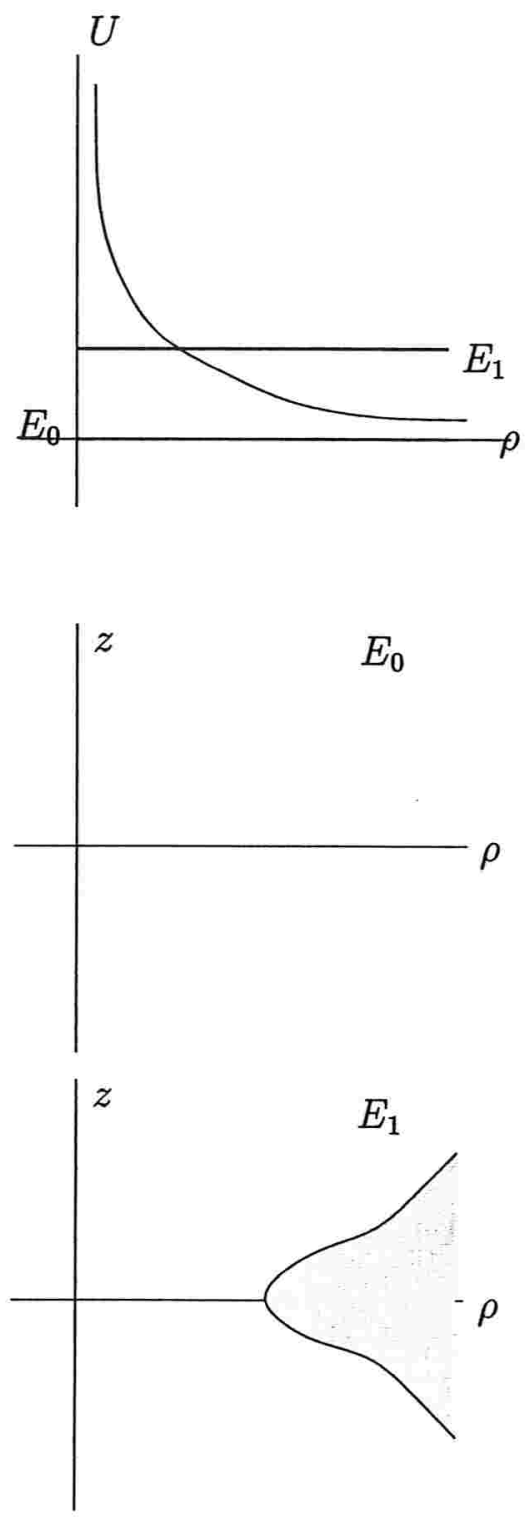

Figura 2.15: Regiões de Hill para o potencial $U$ da região (9) da figura (2.1) abaixo da curva $\Lambda_{2}$ e também para $L<0$. 
Considera-se, então, o caso $L \neq 0$. Com isto, pode-se definir, a partir da equação (2.15), a função

$$
f(r)=2 \beta^{2} r^{5}-9 r^{4}+12 \beta r^{3}+\beta^{2} r^{2}+6 \beta
$$

onde

$$
\beta=\frac{\Lambda}{L}
$$

Desta forma, os zeros de $f(r)$ são os candidatos a pontos críticos fora do plano equatorial. Mais ainda, pode-se obter um valor de $\beta$ para o qual tais possíveis zeros bifurcam de 0 para 2 zeros. Este valor de $\beta$ é tal que $f$ e $f^{\prime}$ se anulam simultaneamente e é aproximadamente

$$
\bar{\beta} \approx 0,92820323 \text {, }
$$

quando $\beta>0$. No caso em que $\beta<0$, os $r$ 's são todos negativos e logo, não são considerados. O caso $\beta=0$ equivale a $\Lambda=0$, e daí $f(r)=-9 r^{4}$, cujo único zero é $r=0$, o qual não é considerado. Observa-se que $L>0$ pois se $L<0$ então $\Lambda<0$ de onde $u<0$, o que é um absurdo. Conclui-se que

- se $0<\beta<\bar{\beta}$ então têm-se dois $r$ 's que satisfazem $f=0$;

- se $\beta=\bar{\beta}$ então tem-se um $r$ que satisfaz $f=0$ e;

- se $\beta>\bar{\beta}$ então não existem zeros de $f=0$.

Resta verificar se para tais candidatos tem-se uma variável $u$ correspondente bem definida. De fato, observa-se que isto só ocorre se a variável $u$ é positiva e se a variável $\rho$, obtida a partir de $u$, é menor que o seu respectivo $r$. Logo, se além de satisfazer $f(r)=0, u$ é tal que

$$
0<u \leq \frac{1}{r}
$$

então o par $(r, u)$ corresponde a um ponto crítico fora do plano equatorial.

Deseja-se determinar que região do plano $(L, \Lambda)$ corresponde a tais pares. Para isso, considera-se novamente as equações (2.12) e (2.13) que determinam os pontos críticos fora do plano $z=0$. Fixando $L$, segue da equação (2.12) que:

$$
u=\frac{L}{\left(1+2 r^{3}\right)^{1 / 2}} .
$$


E da equação (2.13) obtém-se que:

$$
\Lambda=3\left(\frac{(L-u)}{r^{2}}-u r\right)
$$

E por fim como $r^{2}=\rho^{2}+z^{2}$ e $u=\frac{\rho^{2}}{r^{3}}$ resulta que

$$
z=r(1-u r)^{1 / 2}
$$

e portanto através do programa (1) variando $L$ e $r$, é verificado inicialmente se a condição (2.17) é satisfeita pra $u$ dada por (2.18). Caso ela seja verdadeira, calculase $\Lambda$ e $z$ usando as equações (2.19) e (2.20). Se $z$ estiver próximo de zero e $\Lambda$ for positivo, tem-se o par $(L, \Lambda)$. Obtidos numericamente estes pares formam a curva $\chi$ de bifurcação entre 1 par de zeros e 2 pares de zeros fora do plano equatorial, o que pode ser visto na figura (2.16). Observa-se que tais pontos críticos aparecem aos pares, um simétrico ao outro em relação ao eixo $z=0$.

Assim, classificando-se tais pontos de equilíbrio, as regiões de Hill podem ser esboçadas. A fim de obter tal classificação, lineariza-se o sistema (1.7), obtendo-se

$$
\zeta^{\prime}=\mathbf{M} \zeta
$$

onde $\zeta=\left(\rho, \phi, z, p_{\rho}, L, p_{z}\right)$

$$
\mathbf{M}=\left(\begin{array}{cccccc}
0 & 0 & 0 & H_{p_{\rho} p_{\rho}} & 0 & 0 \\
H_{L \rho} & 0 & H_{L z} & 0 & H_{L L} & 0 \\
0 & 0 & 0 & 0 & 0 & H_{p_{z} p_{z}} \\
-H_{\rho \rho} & 0 & -H_{\rho z} & 0 & -H_{\rho L} & 0 \\
0 & 0 & 0 & 0 & 0 & 0 \\
-H_{z \rho} & 0 & -H_{z z} & 0 & -H_{z L} & 0
\end{array}\right)
$$

e $H_{i j}$ são as derivadas segundas do Hamiltoniano $H$ no ponto crítico $\zeta_{*}$, $\operatorname{com} i, j=$ $\rho, L, z, p_{\rho}, p_{z}$.

O polinômio característico de $\mathbf{M}$ é dado por:

$$
p(\lambda)=\lambda^{2}\left(\lambda^{4}+\left(H_{\rho \rho}+H_{z z}\right) \lambda^{2}+\left(H_{\rho \rho} H_{z z}-H_{\rho z}^{2}\right)\right)
$$

onde, em $\zeta_{*}$,

$$
H_{\rho \rho}=\frac{4 L^{2}}{\rho_{*}^{4}}-\frac{3 \Lambda \rho_{*}^{2}}{\left(\rho_{*}^{2}+z_{*}^{2}\right)^{5 / 2}}+3 \rho_{*}^{2}\left(\frac{\rho_{*}^{2}-4 z_{*}^{2}-5 L}{\left(\rho_{*}^{2}+z_{*}^{2}\right)^{7 / 2}}\right)+\frac{12 \rho_{*}^{2}\left(\rho_{*}^{2}-z_{*}^{2}\right)}{\left(\rho_{*}^{2}+z_{*}^{2}\right)^{5}}
$$




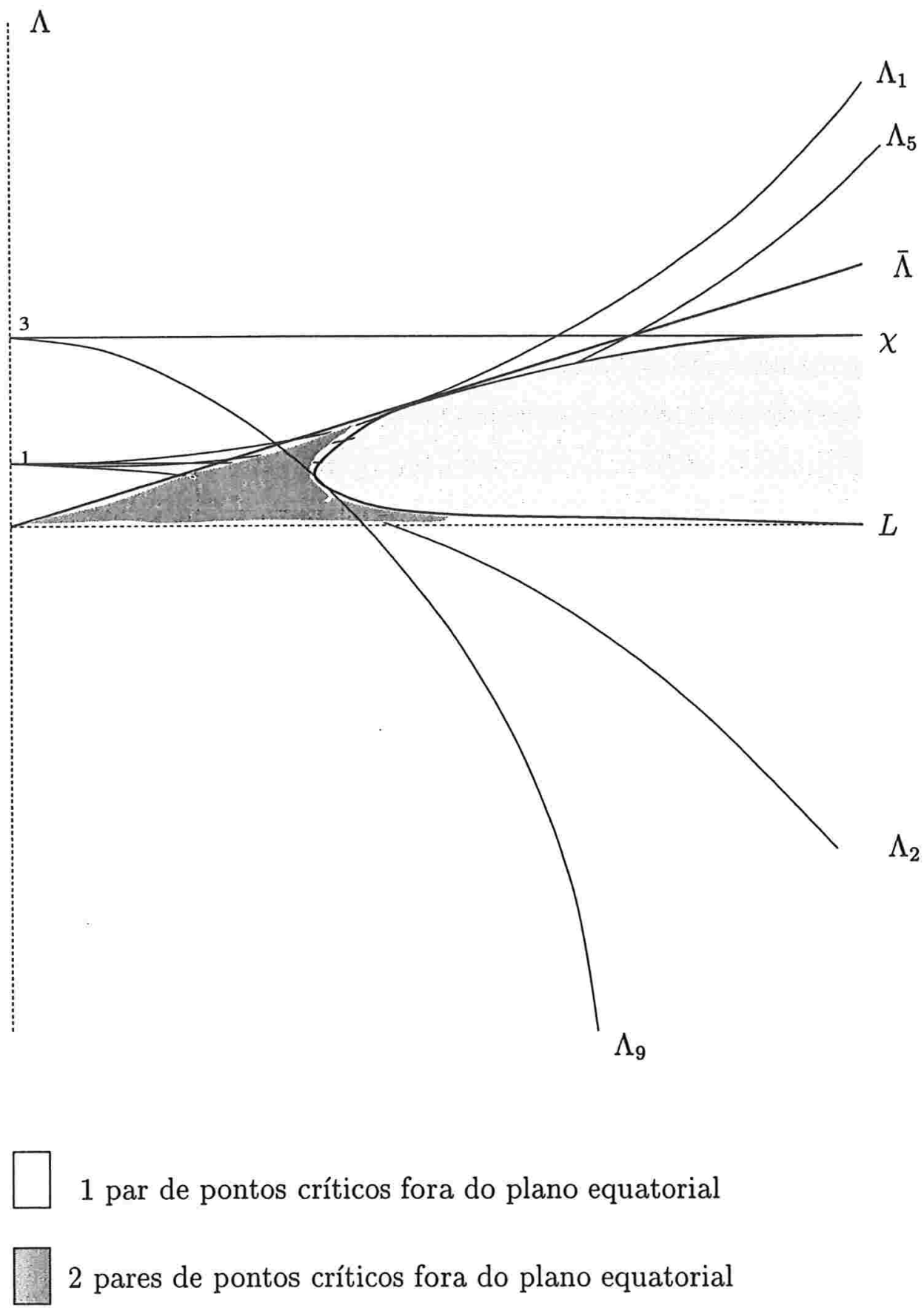

Figura 2.16: Diagrama de bifurcação do número de zeros fora do plano equatorial em termos do par $(L, \Lambda)$ para $Q \mu>0$ onde $\Lambda_{1}, \Lambda_{2}, \Lambda_{5}$ são como na figura (1.1), $\Lambda_{9}$ é como na figura (1.3), $\bar{\Lambda}=0,92820323 L$ e $\chi$ obtida numericamente, conforme explicado nesta seção. 


$$
H_{z z}=3 z^{2}\left(-\frac{\Lambda}{\left(\rho_{*}^{2}+z_{*}^{2}\right)^{5 / 2}}+\frac{5\left(\rho^{2}-L\right)}{\left(\rho_{*}^{2}+z_{*}^{2}\right)^{7 / 2}}+\frac{8 \rho_{*}}{\left(\rho_{*}^{2}+z_{*}^{2}\right)^{5}}\right)
$$

e

$$
H_{\rho z}=-3 \rho_{*} z_{*}\left(\frac{\Lambda}{\left(\rho_{*}^{2}+z_{*}^{2}\right)^{5 / 2}}+\frac{5 L+2 z_{*}^{2}-3 \rho_{*}^{2}}{\left(\rho_{*}^{2}+z_{*}^{2}\right)^{7 / 2}}+\frac{2\left(z_{*}^{2}-3 \rho_{*}^{2}\right)}{\left(\rho_{*}^{2}+z_{*}^{2}\right)^{5}}\right)
$$

Após o cálculo para diversos casos conclui-se que tais pontos de equilíbrio são de dois tipos: centro-centro e sela-centro, sendo que o segundo só aparece no caso onde temos dois pares de pontos críticos fora do plano equatorial.

Neste momento esboçam-se as regiões de Hill para os casos onde existem pontos de equilíbrio fora do plano equatorial. São elas: as regiões (1B), (2B), (3A), (3B), $(4 \mathrm{~A}),(4 \mathrm{~B}),(5 \mathrm{~A} 1),(5 \mathrm{~A} 2),(5 \mathrm{~A} 3),(5 \mathrm{~B}),(6 \mathrm{~A}),(6 \mathrm{~B}),(7 \mathrm{~A}),(7 \mathrm{~B})$ das figuras $(2.1) \mathrm{e}$ (2.2).

Observa-se que nos casos A tem-se o par de equilíbrios do tipo centro-centro e, nos casos B têm-se os dois pares do tipo centro-centro e sela-centro. Nas regiões (3), (4), (5), (6) e (7), o equilíbrio do tipo centro-centro de energia $E_{3}$ se bifurca em dois formando o par do tipo centro-centro que aparece nas regiões $\mathrm{A}$. O par de equilíbrios do tipo centro-sela que aparece nas regiões B se origina da bifurcação do equilíbrio do tipo sela-centro das regiões A. Sobre a curva $\chi$, estes pares coincidem com os equilíbrios no plano equatorial.

1. Região 1B: $L>0$ e $\beta_{2} L^{3}+1<\Lambda<\bar{\beta} L$ (Figura 2.17)

- Existência da região "salsicha", de acordo com o caso (1) da seção (2.2).

- O potencial possui apenas um ponto crítico do tipo centro-sela $\left(E_{1}\right)$ no plano equatorial e dois pares de pontos críticos fora deste plano, sendo: dois do tipo centro-centro $\left(E_{3}\right)$ e dois do tipo sela-centro $\left(E_{5}\right)$.

2. Região 2B: $L>0$ e $\Lambda=\beta_{2} L^{3}+1$ e $\Lambda<\bar{\beta} L$ (Figura 2.18)

- Existência da região "salsicha", de acordo com o caso (1) da seção (2.2).

- O potencial possui dois pontos críticos no plano equatorial, sendo: um do tipo centro-sela(energia $E_{1}$ ) e outro do tipo parabólico-sela(energia $E_{7}$ ) e, mais dois pares de pontos críticos fora deste plano, sendo: dois do tipo centro-centro $\left(E_{3}\right)$ e dois do tipo sela-centro $\left(E_{5}\right)$. 

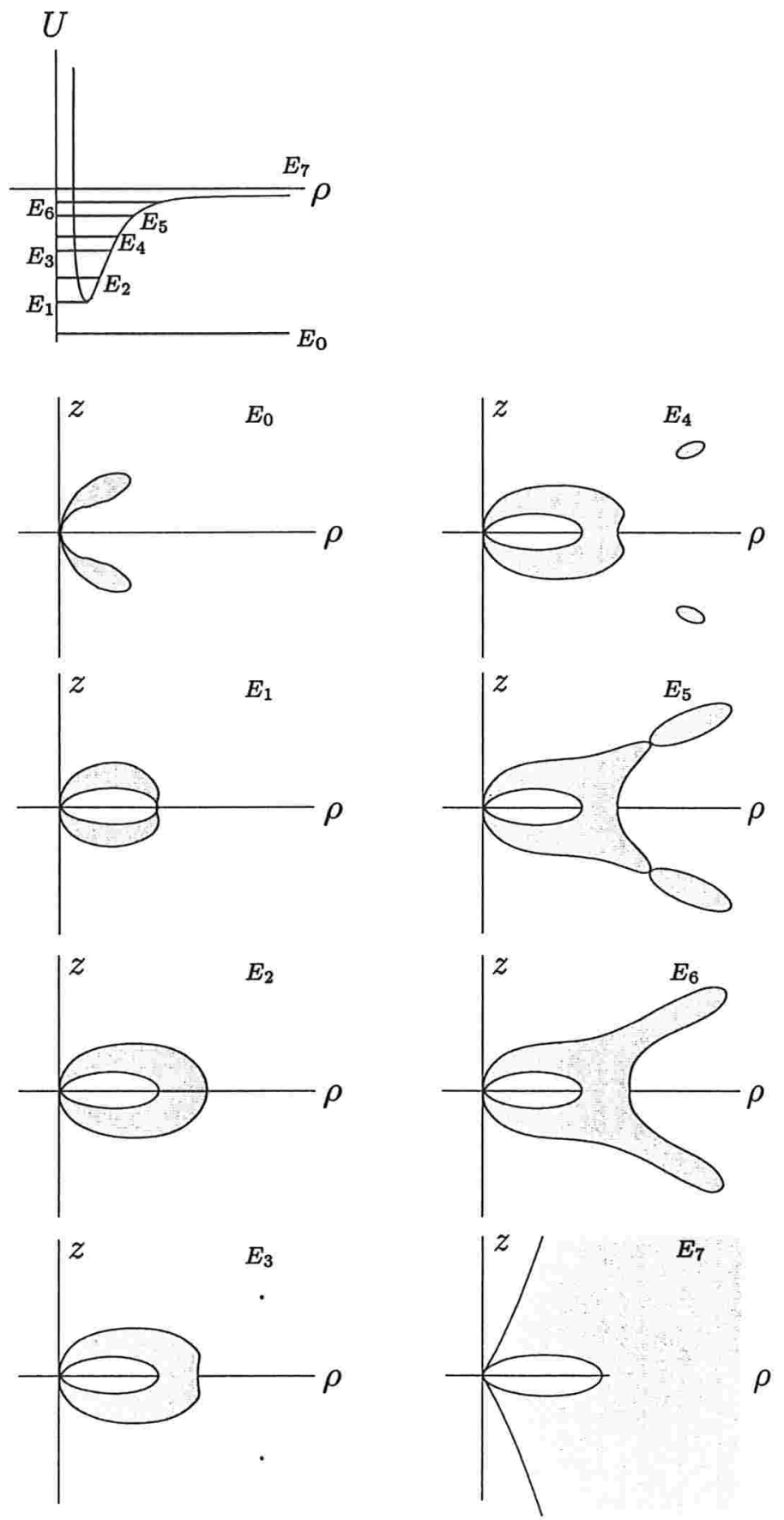

Figura 2.17: Regiões de Hill para o potencial $U$ da região (1B) da figura (2.2) acima da curva $\Lambda_{2}$. 

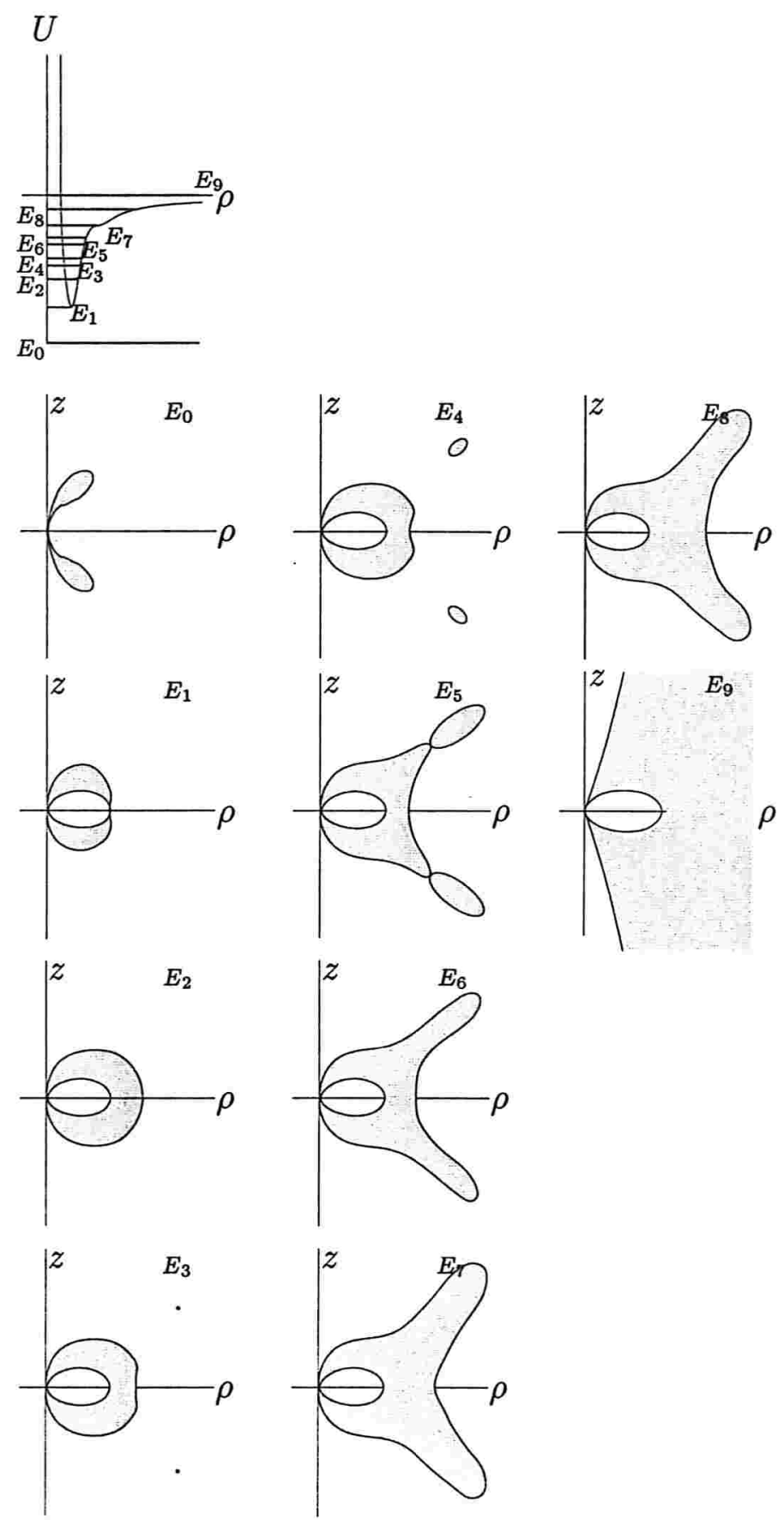

Figura 2.18: Regiões de Hill para o potencial $U$ da região (2B) da figura (2.2) sobre a curva $\Lambda_{2}$. 
3. Região 3A: $L>0$ e $\beta_{2} L^{3}+1<\Lambda<\beta_{6} L^{3}+1$ e $\Lambda<\bar{\beta} L$ e $(L, \Lambda) \in \operatorname{int}(\chi)$ (Figura 2.19)

- Existência da região "salsicha", de acordo com o caso (1) da seção (2.2).

- O potencial possui três pontos críticos no plano equatorial, sendo: um do tipo centro-sela(energia $E_{1}$ ), outro do tipo sela-centro(energia $E_{7}$ ) e um outro do tipo centro-sela(energia $E_{5}$ ) e, mais um par de pontos críticos fora deste plano do tipo centro-centro $\left(E_{3}\right)$.

4. Região 3B: $L>0$ e $\beta_{2} L^{3}+1<\Lambda<\beta_{6} L^{3}+1$ e $\Lambda<\bar{\beta} L$ (Figura 2.20)

- Existência da região "salsicha", de acordo com o caso (1) da seção (2.2).

- O potencial possui três pontos críticos no plano equatorial, sendo: um do tipo centro-sela(energia $E_{1}$ ), outro do tipo sela-sela(energia $E_{9}$ ) e um outro do tipo centro-sela(energia $E_{5}$ ) e, mais dois pares de pontos críticos fora deste plano, sendo: dois do tipo centro-centro $\left(E_{3}\right)$ e dois do tipo sela-centro $\left(E_{7}\right)$.

5. Região 4A: $L>0$ e $\Lambda=\beta_{6} L^{3}+1$ e $\Lambda<\bar{\beta} L$ e $(L, \Lambda) \in \operatorname{int}(\chi)$ (Figura 2.21)

- Existência da região "salsicha", de acordo com o caso (1) da seção (2.2).

- O potencial possui três pontos críticos no plano equatorial, sendo: um do tipo centro-sela(energia $E_{1}$ ), outro do tipo sela-centro(energia $E_{7}$ ) e um outro do tipo centro-sela(energia $E_{5}$ ) e, mais um par de pontos críticos fora deste plano do tipo centro-centro $\left(E_{3}\right)$.

- Este caso difere do caso da região (3A) pelo fato de que a energia do sela-centro $\left(E_{7}\right)$ coincide com a energia 0 .

6. Região 4B: $L>0$ e $\Lambda=\beta_{6} L^{3}+1$ e $\Lambda<\bar{\beta} L$ (Figura 2.22)

- Existência da região "salsicha", de acordo com o caso (1) da seção (2.2).

- O potencial possui três pontos críticos no plano equatorial, sendo: um do tipo centro-sela(energia $E_{1}$ ), outro do tipo sela-sela(energia $E_{9}$ ) e um outro do tipo centro-sela(energia $\left.E_{5}\right)$ e, mais dois pares de pontos críticos 

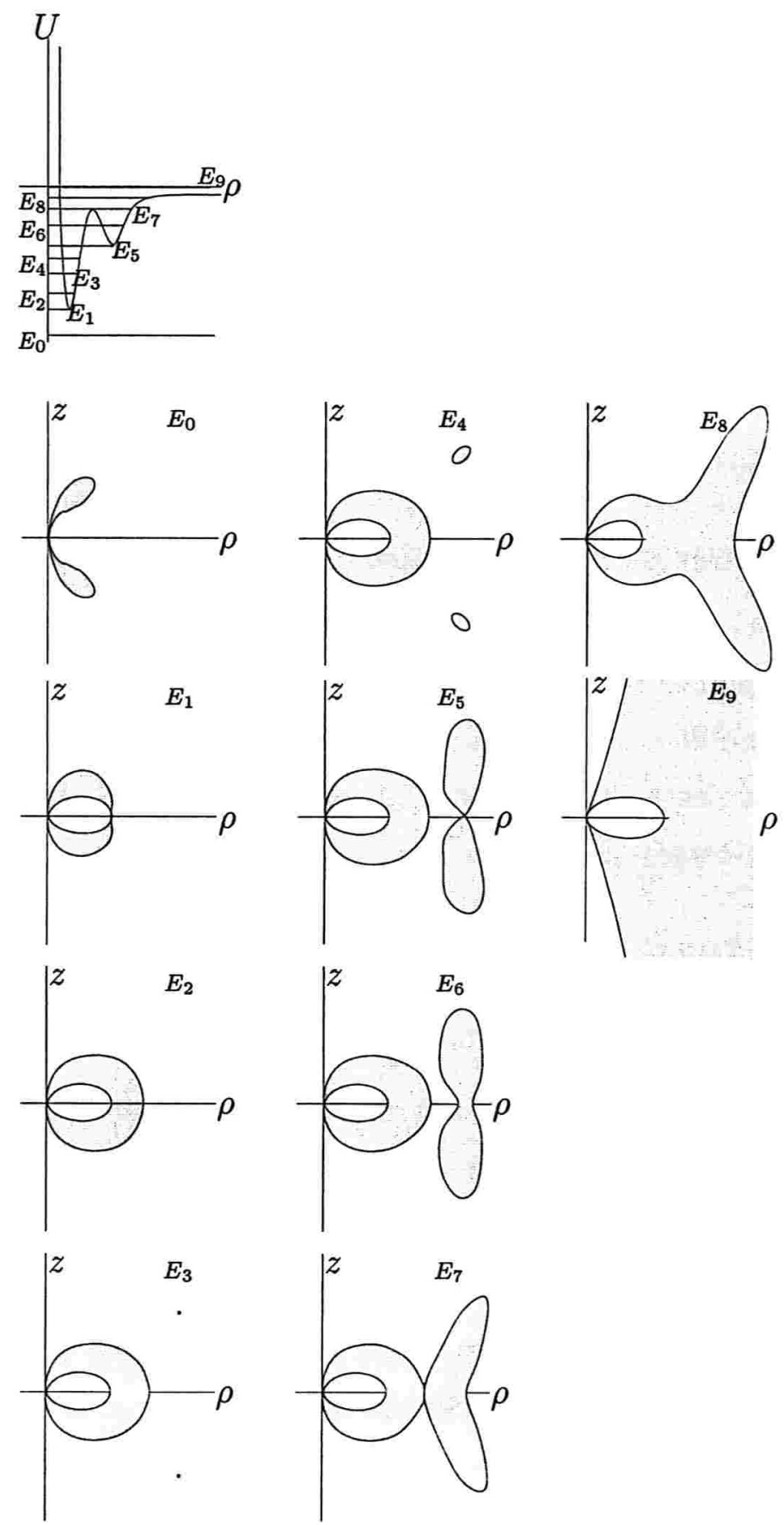

Figura 2.19: Regiões de Hill para o potencial $U$ da região (3A) da figura (2.2) entre as curvas $\Lambda_{2}$ e $\Lambda_{6}$. 

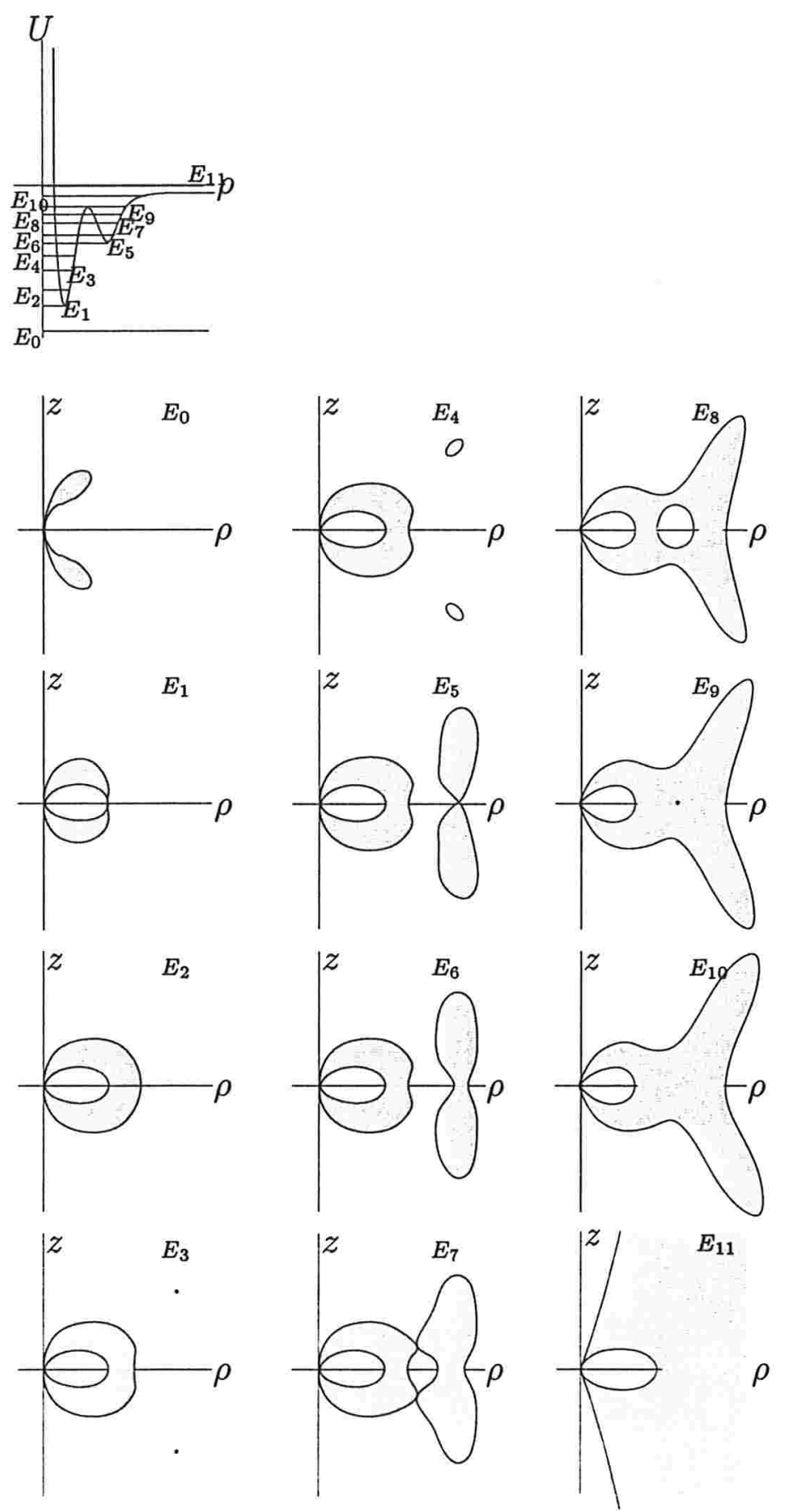

Figura 2.20: Regiões de Hill para o potencial $U$ da região (3B) da figura (2.2) entre as curvas $\Lambda_{2}$ e $\Lambda_{6}$. 

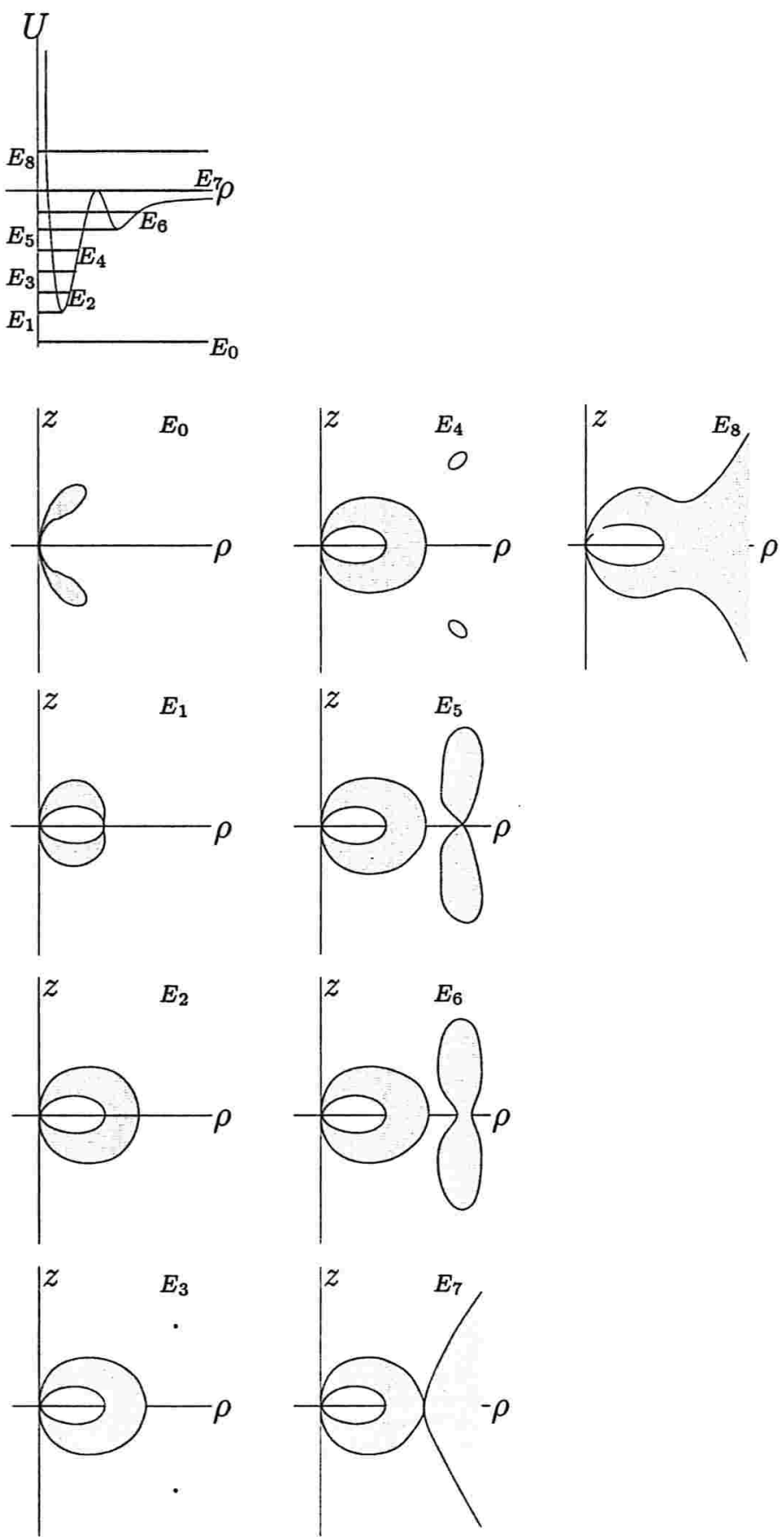

Figura 2.21: Regiões de Hill para o potencial $U$ da região (4A) da figura (2.2) sobre a curva $\Lambda_{6}$. 
fora deste plano, sendo: dois do tipo centro-centro $\left(E_{3}\right)$ e dois do tipo sela-centro $\left(E_{7}\right)$.

- Este caso difere do caso da região (3B) pelo fato de que a energia do sela-sela $\left(E_{9}\right)$ coincide com a energia 0 .

No caso da região (5A) da figura (2.1) têm-se três casos a considerar onde a topologia das regiões de Hill se modifica dependendo das energias dos dois poços do potencial $U$.

7. Região 5A1: $L>0$ e $\beta_{6} L^{3}+1<\Lambda<1$ e $\Lambda>$ e $(L, \Lambda) \in \operatorname{int}(\chi)$ (Figura 2.23)

- Existência da região "salsicha", de acordo com o caso (1) da seção (2.2).

- O potencial possui três pontos críticos no plano equatorial, sendo: um do tipo centro-sela(energia $E_{1}$ ), outro do tipo sela-centro(energia $E_{7}$ ) e um outro do tipo centro-sela(energia $E_{5}$ ) e, mais um par de pontos críticos fora deste plano do tipo centro-centro $\left(E_{3}\right)$.

- Neste caso, diferentemente dos casos das regiões (3A) e (4A), a energia do sela-centro $\left(E_{8}\right)$ é maior que a energia $E_{7}=0$.

- A energia $E_{1}$ do primeiro poço é menor que a energia $E_{5}$ do segundo poço.

8. Região 5A2: $L>0$ e $\beta_{6} L^{3}+1<\Lambda<1$ e $\Lambda>$ e $(L, \Lambda) \in \operatorname{int}(\chi)$ (Figura 2.24)

- Existência da região "salsicha", de acordo com o caso (1) da seção (2.2).

- O potencial possui três pontos críticos no plano equatorial, sendo: um do tipo centro-sela(energia $E_{3}$ ), outro do tipo sela-centro(energia $E_{6}$ ) e um outro do tipo centro-sela(energia $E_{3}$ ) e, mais um par de pontos críticos fora deste plano do tipo centro-centro $\left(E_{1}\right)$.

- Neste caso, diferentemente dos casos das regiões (3A) e (4A), a energia do sela-centro $\left(E_{6}\right)$ é maior que a energia $E_{5}=0$.

- As energias dos dois poços coincidem $\left(E_{3}\right)$. 

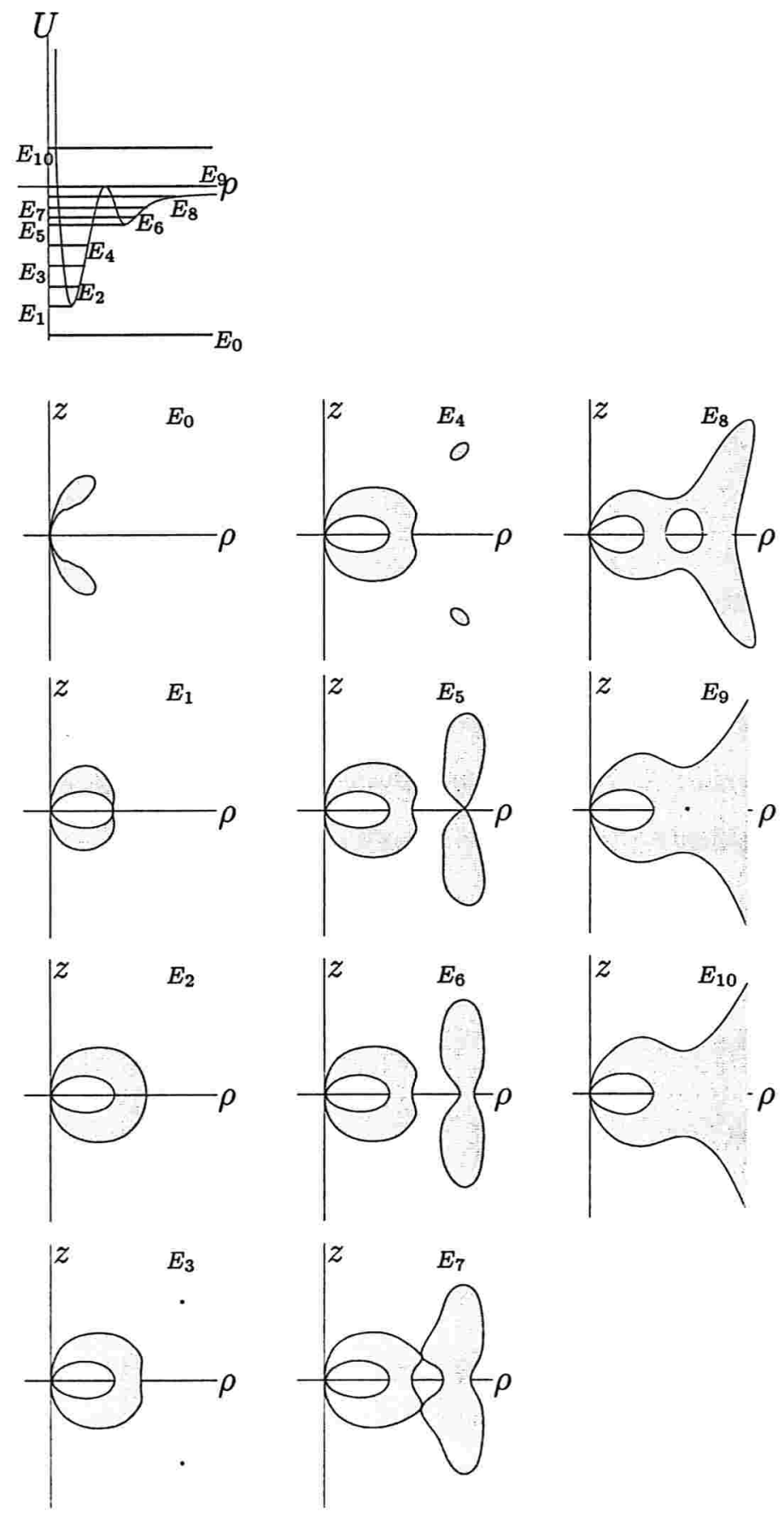

Figura 2.22: Regiões de Hill para o potencial $U$ da região (4B) da figura (2.2) sobre a curva $\Lambda_{6}$. 

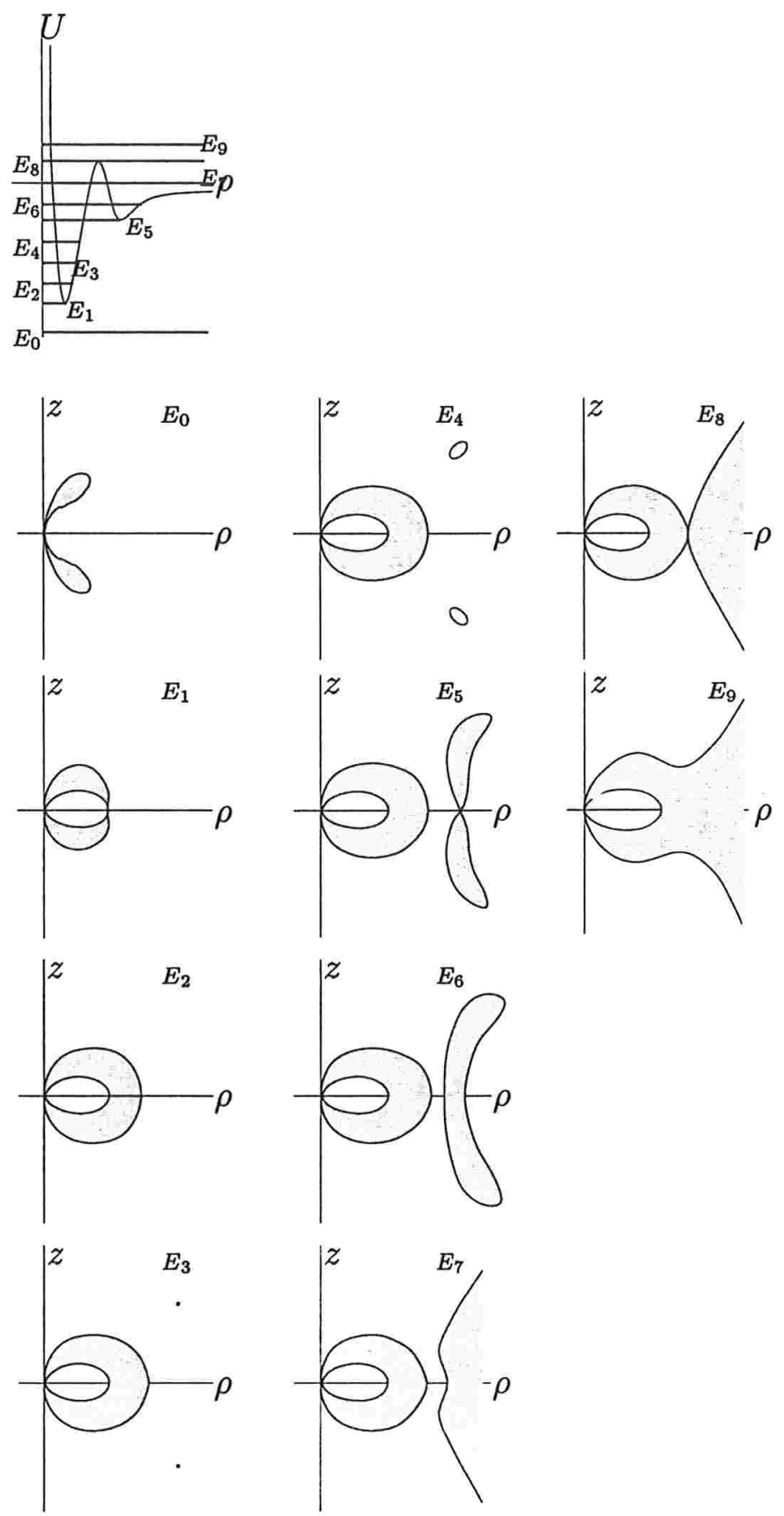

Figura 2.23: Regiões de Hill para o potencial $U$ da região (5A1) da figura (2.1) entre as curvas $\Lambda_{6}$ e $\Lambda=1$. 

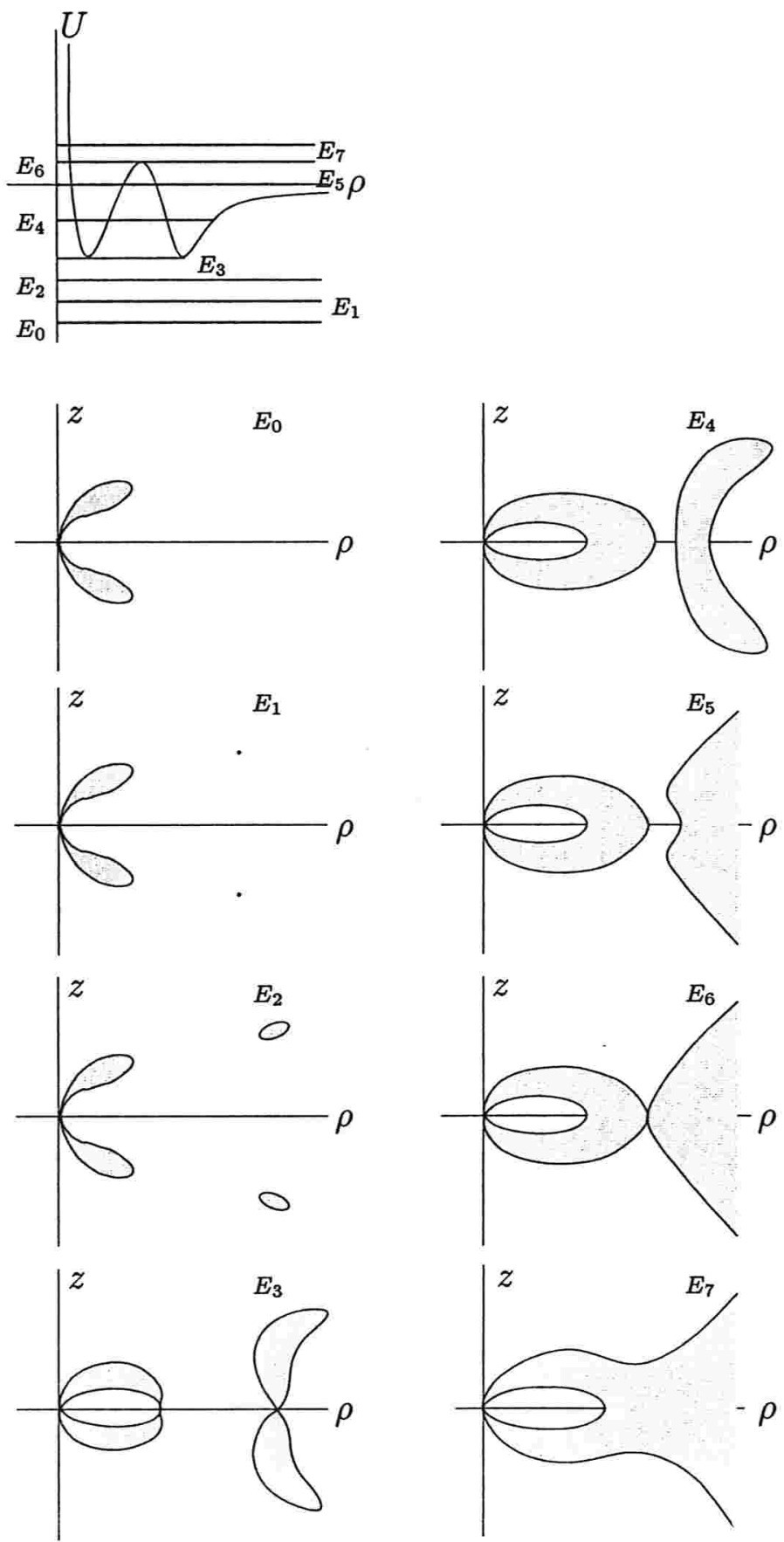

Figura 2.24: Regiões de Hill para o potencial $U$ da região (5A2) da figura (2.1) entre as curvas $\Lambda_{6}$ e $\Lambda=1$. 
9. Região 5A3: $L>0$ e $\beta_{6} L^{3}+1<\Lambda<1$ e $\Lambda>$ e $(L, \Lambda) \in \operatorname{int}(\chi)$ (Figura 2.25)

- Existência da região "salsicha", de acordo com o caso (1) da seção (2.2).

- O potencial possui três pontos críticos no plano equatorial, sendo: um do tipo centro-sela(energia $E_{5}$ ), outro do tipo sela-centro(energia $E_{8}$ ) e um outro do tipo centro-sela(energia $E_{3}$ ) e, mais um par de pontos críticos fora deste plano do tipo centro-centro $\left(E_{1}\right)$.

- Neste caso, diferentemente dos casos das regiões (3A) e (4A), a energia do sela-centro $\left(E_{8}\right)$ é maior que a energia $E_{7}=0$.

- A energia $E_{5}$ do primeiro poço é maior que a energia $E_{3}$ do segundo poço.

10. Região 5B: $L>0$ e $\beta_{6} L^{3}+1<\Lambda<1$ e $\Lambda<\bar{\beta} L$ (Figura 2.26)

- Existência da região "salsicha", de acordo com o caso (1) da seção (2.2).

- O potencial possui três pontos críticos no plano equatorial, sendo: um do tipo centro-sela(energia $E_{1}$ ), outro do tipo sela-sela(energia $E_{1} 0$ ) e um outro do tipo centro-sela(energia $E_{5}$ ) e, mais dois pares de pontos críticos fora deste plano, sendo: dois do tipo centro-centro $\left(E_{3}\right)$ e dois do tipo sela-centro $\left(E_{8}\right)$.

- Neste caso, diferentemente dos casos das regiões (3B) e (4B), a energia do sela-centro $\left(E_{1} 0\right)$ é maior que a energia $E_{7}=0$.

11. Região 6A: $L>0$ e $\Lambda=1$ e $\Lambda<\bar{\beta} L$ e $(L, \Lambda) \in \operatorname{int}(\chi)$ (Figura 2.27)

- Existência da região "salsicha", de acordo com o caso (1) da seção (2.2).

- O potencial possui dois pontos críticos no plano equatorial, sendo: um do tipo centro-sela(energia $E_{3}=0$ ) e outro do tipo sela-centro(energia $E_{5}$ ) e, mais um par de pontos críticos fora deste plano do tipo centro-centro $\left(E_{1}\right)$.

12. Região 6B: $L>0$ e $\Lambda=1$ e $\Lambda<\bar{\beta} L$ (Figura 2.28)

- Existência da região "salsicha", de acordo com o caso (1) da seção (2.2). 

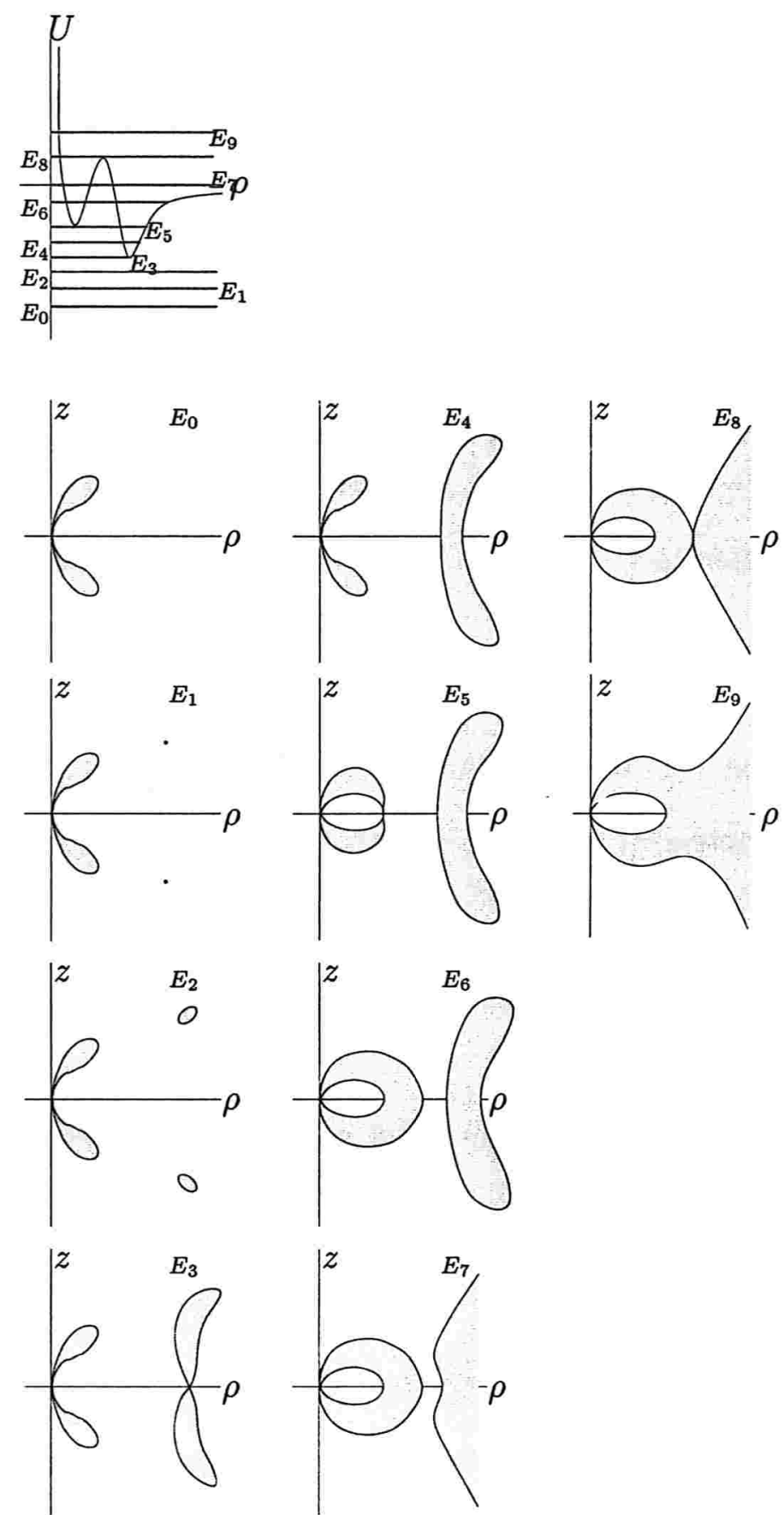

Figura 2.25: Regiões de Hill para o potencial $U$ da região (5A3) da figura (2.1) entre as curvas $\Lambda_{6}$ e $\Lambda=1$. 

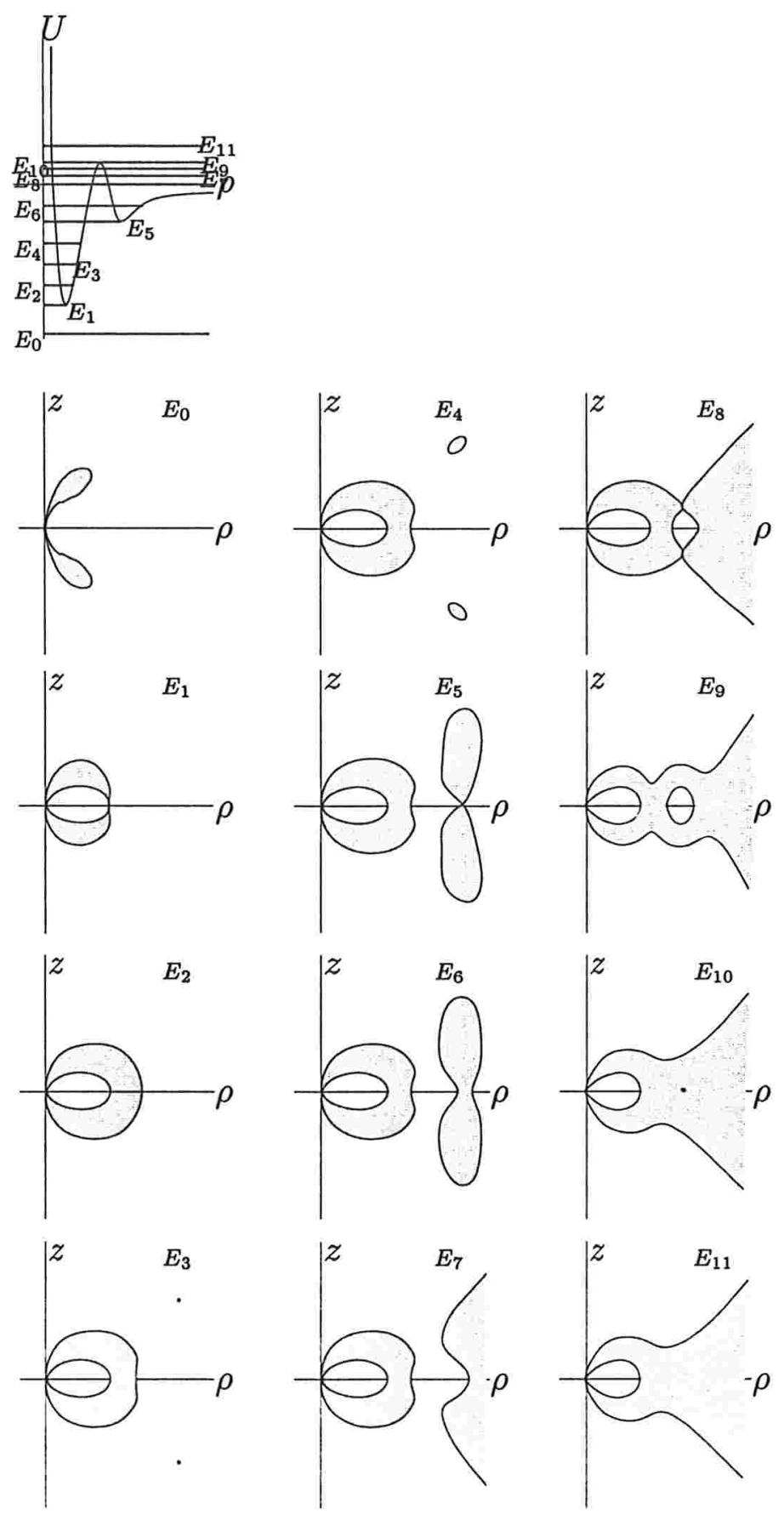

Figura 2.26: Regiões de Hill para o potencial $U$ da região (5B) da figura (2.2) entre as curvas $\Lambda_{6}$ e $\Lambda=1$. 

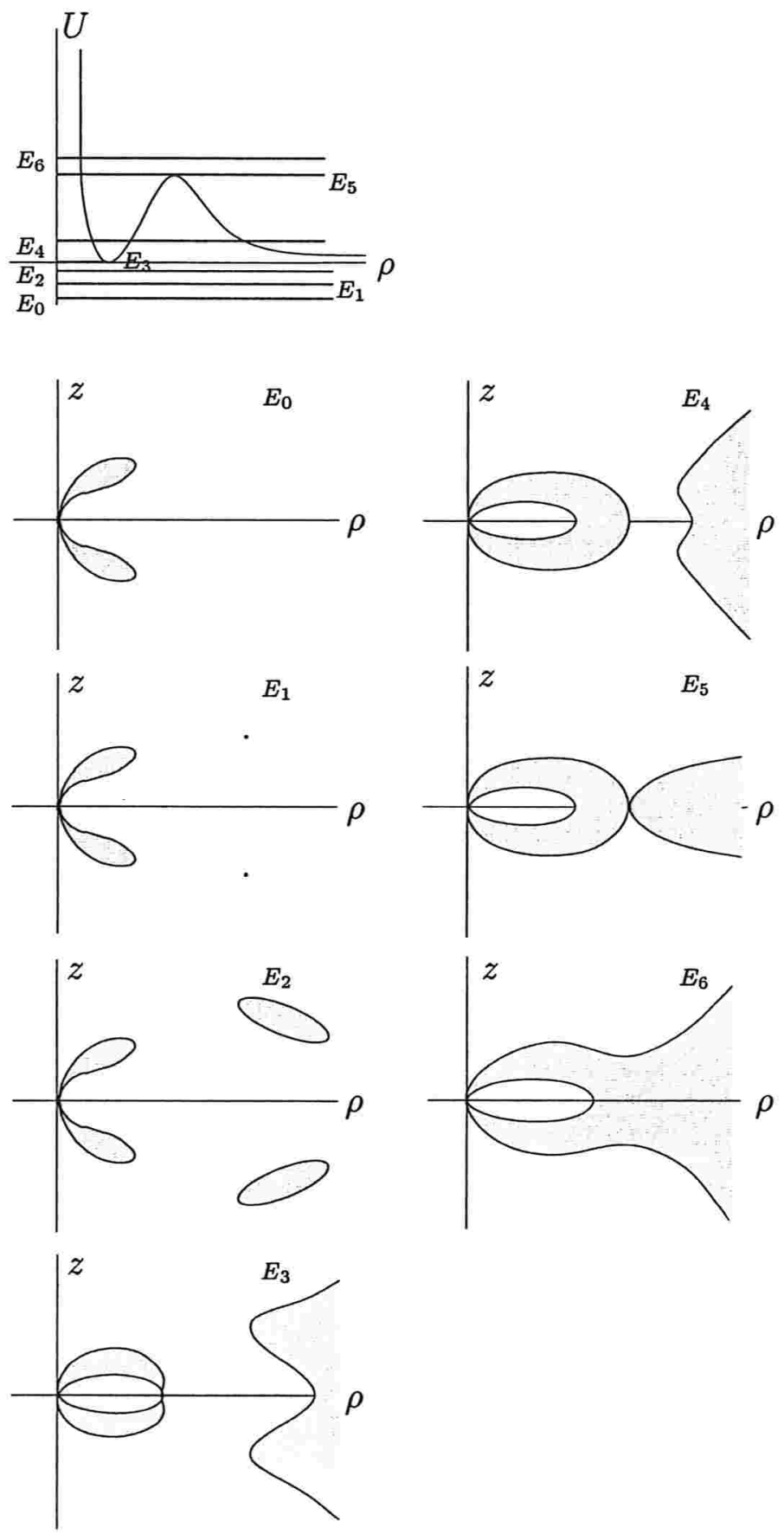

Figura 2.27: Regiões de Hill para o potencial $U$ da região (6A) da figura (2.1) sobre a curva $\Lambda=1$. 
- O potencial possui dois pontos críticos no plano equatorial, sendo: um do tipo centro-sela(energia $E_{3}=0$ ) e outro do tipo sela-sela(energia $E_{7}$ ) e, mais dois pares de pontos críticos fora deste plano, sendo: dois do tipo centro-centro $\left(E_{1}\right)$ e dois do tipo sela-centro $\left(E_{5}\right)$.

13. Região 7A: $L>0$ e $1<\Lambda<\beta_{1} L^{3}+1$ e $\Lambda<\bar{\beta} L$ e $(L, \Lambda) \in \operatorname{int}(\chi)$ (Figura 2.29)

- Existência da região "salsicha", de acordo com o caso (1) da seção (2.2).

- O potencial possui dois pontos críticos no plano equatorial, sendo: um do tipo centro-sela(energia $E_{4}$ ) e outro do tipo sela-centro(energia $E_{6}$ ) e, mais um par de pontos críticos fora deste plano do tipo centro-centro $\left(E_{1}\right)$.

- Este caso difere do caso da região (6A) pelo fato de que a energia do centro-sela $\left(E_{4}\right)$ é maior do que 0 .

14. Região 7B: $L>0$ e $1<\Lambda<\beta_{1} L^{3}+1$ e $\Lambda<\bar{\beta} L$ (Figura 2.30)

- Existência da região "salsicha", de acordo com o caso (1) da seção (2.2).

- O potencial possui dois pontos críticos no plano equatorial, sendo: um do tipo centro-sela(energia $E_{4}$ ) e outro do tipo sela-sela(energia $E_{8}$ ) e, mais dois pares de pontos críticos fora deste plano, sendo: dois do tipo centro-centro $\left(E_{1}\right)$ e dois do tipo sela-centro $\left(E_{6}\right)$.

- Este caso difere do caso da região (6B) pelo fato de que a energia do centro-sela $\left(E_{4}\right)$ é maior do que 0 . 

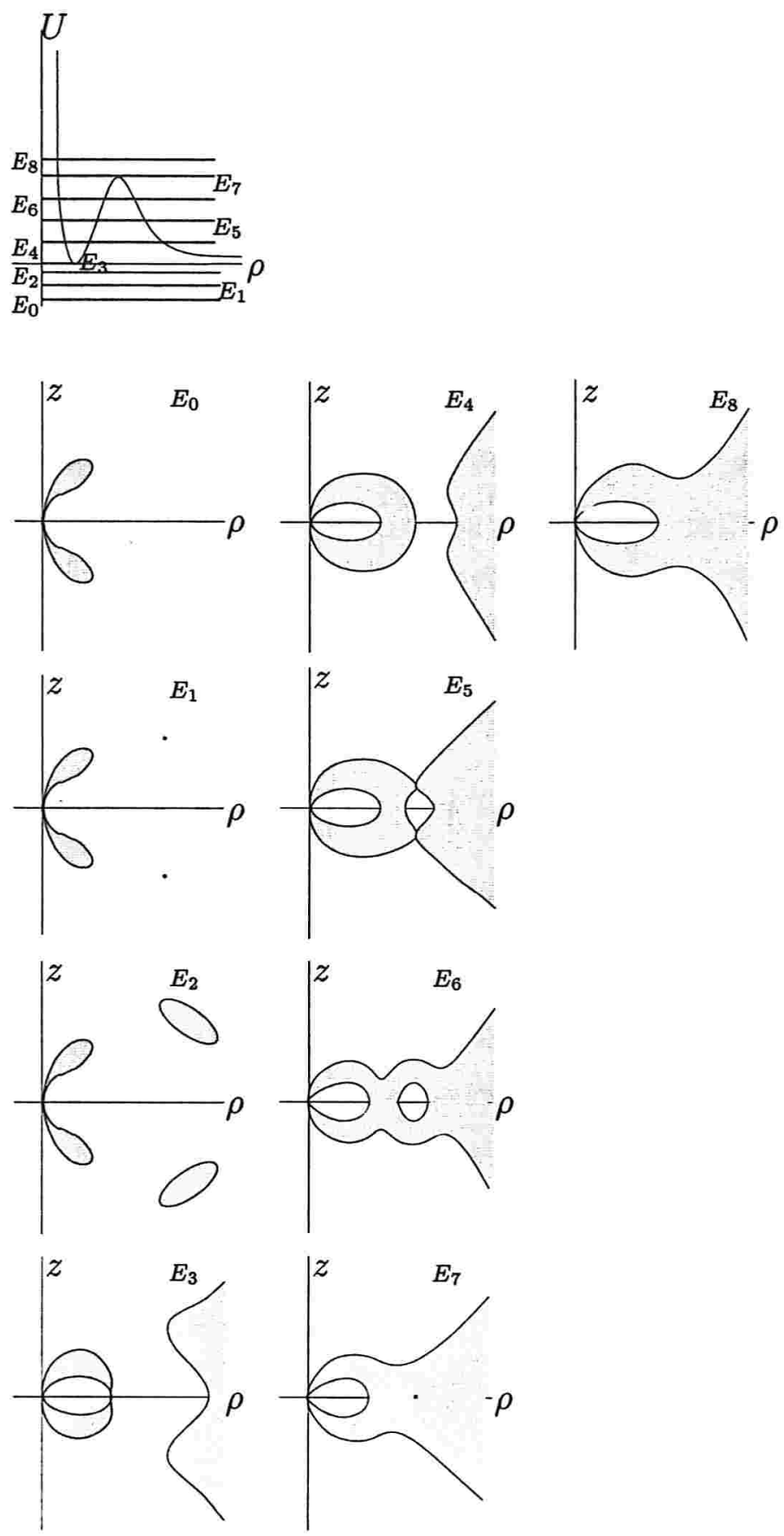

Figura 2.28: Regiões de Hill para o potencial $U$ da região (6B) da figura (2.2) sobre a curva $\Lambda=1$. 

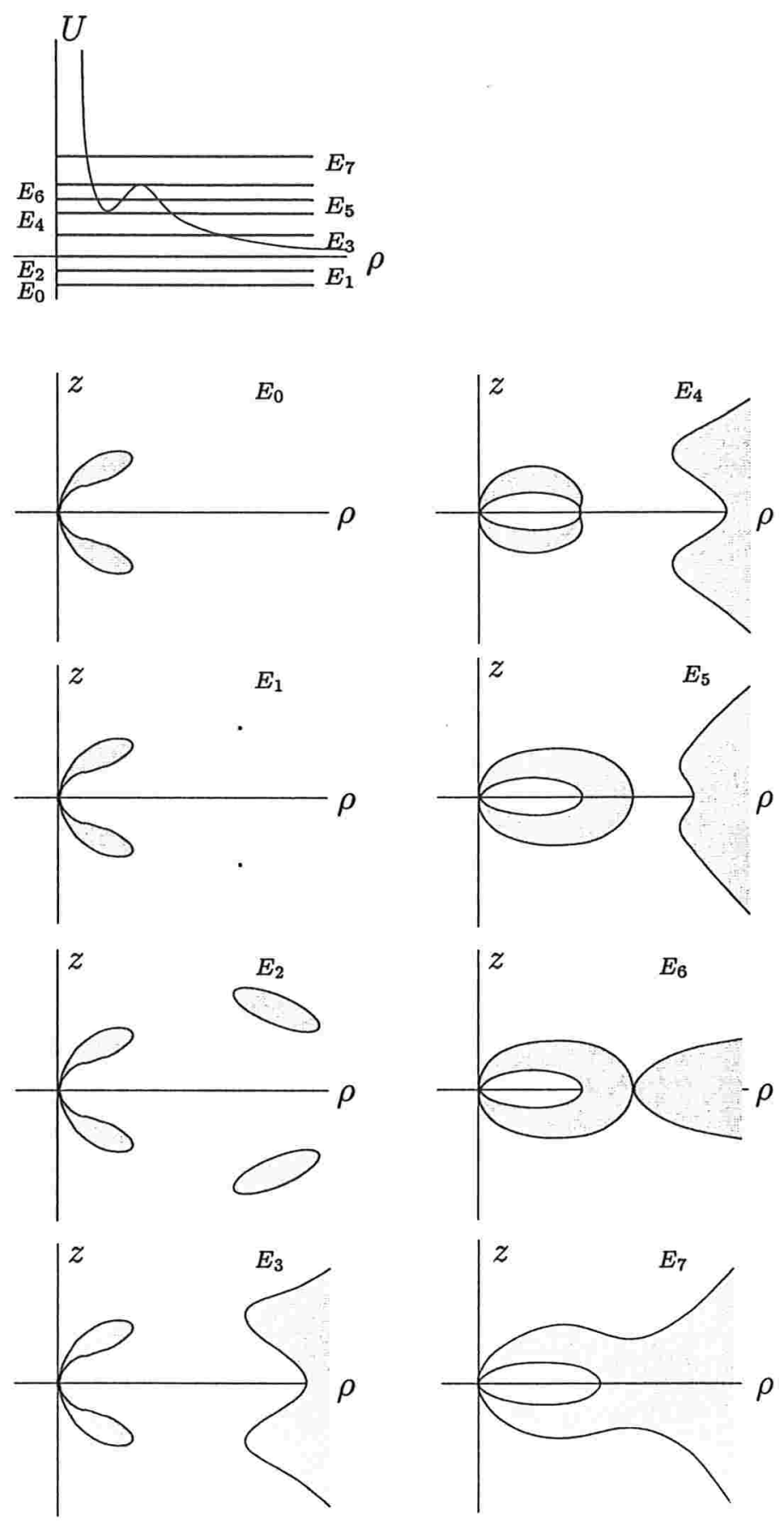

Figura 2.29: Regiões de Hill para o potencial $U$ da região (7A) da figura (2.1) entre as curvas $\Lambda=1$ e $\Lambda_{1}$. 

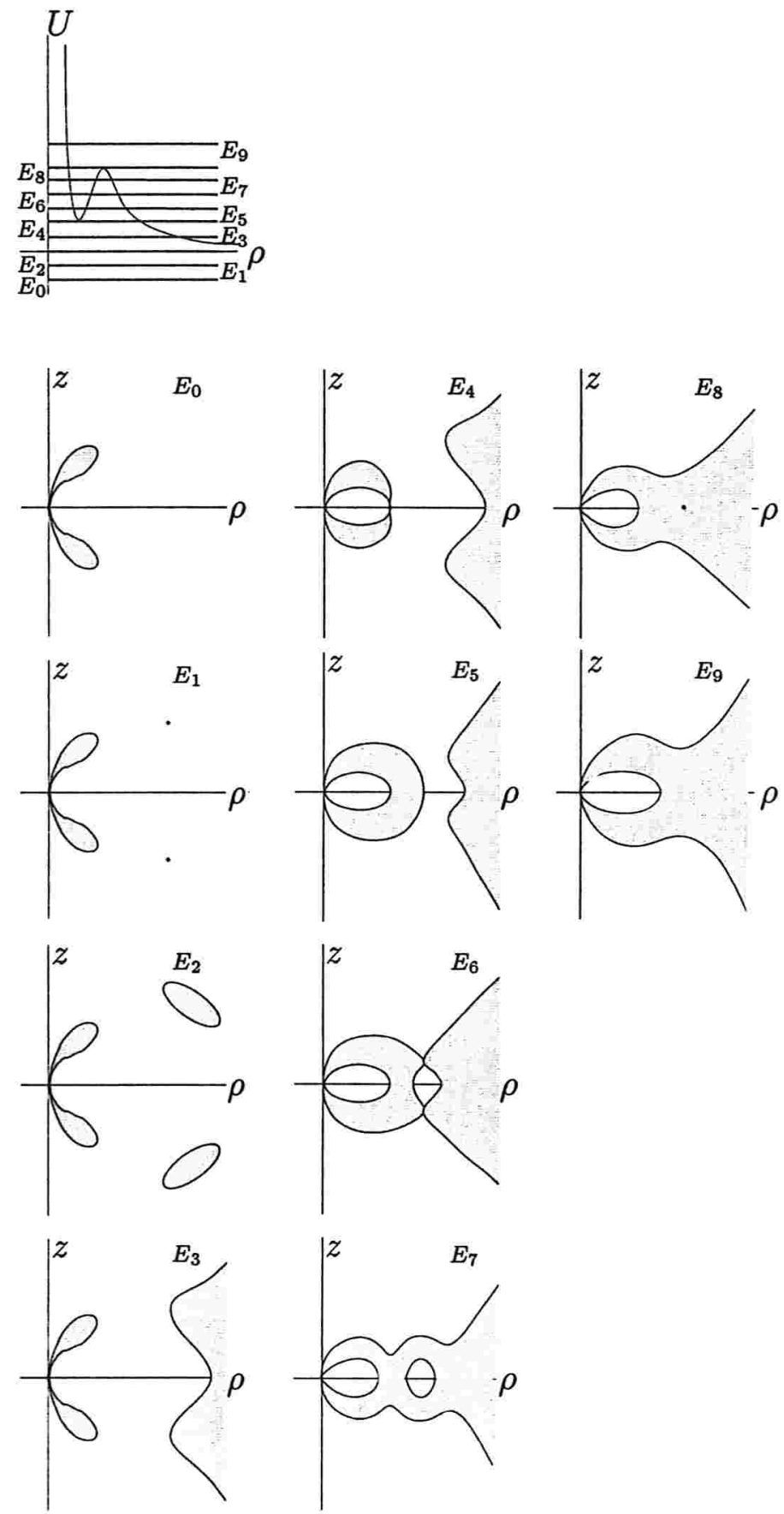

Figura 2.30: Regiões de Hill para o potencial $U$ da região (7B) da figura (2.2) entre as curvas $\Lambda=1$ e $\Lambda_{1}$. 
CAPÍTULO 3

\section{Dinâmica Próxima às Órbitas Homoclínicas ao Sela-Centro}

Neste último capítulo apresenta-se um breve estudo da dinâmica próxima aos laços homoclínicos sela-centro. Na primeira seção é introduzida a aplicação de Poincaré e uma aproximação discreta da mesma. Esta aproximação possui dois invariantes: $\gamma$ e $\alpha$. O invariante $\gamma$ está associado aos autovalores do sela-centro. O invariante $\alpha$ é definido na seção seguinte, onde também é dada uma interpretação geométrica para ele. Na seção 3, apresenta-se o algoritmo usado para o cálculo numérico do invariante $\alpha$. Discorre-se um pouco sobre a estabilidade orbital dos laços sela-centro na seção 4. E finalmente, na última seção, mostra-se a existência de atratores para a aproximação discreta apresentada na seção inicial.

\subsection{Aplicação de Poincaré e sua Aproximação Dis- creta}

Neste momento, pretende-se estudar a dinâmica próxima às órbitas homoclínicas ao equilíbrio sela-centro contidas no plano equatorial. Duas tais órbitas são mostradas na figura (3.1). Para tanto, considera-se o conjunto invariante dado pelo equilíbrio sela-centro e pela órbita homoclínica a ele, o qual é denominado laço sela-centro e denotado por $\Gamma$. Observa-se que, do ponto de vista topológico, um laço sela-centro é similar a uma órbita periódica de "período infinito", o que sugere o uso de seções de 

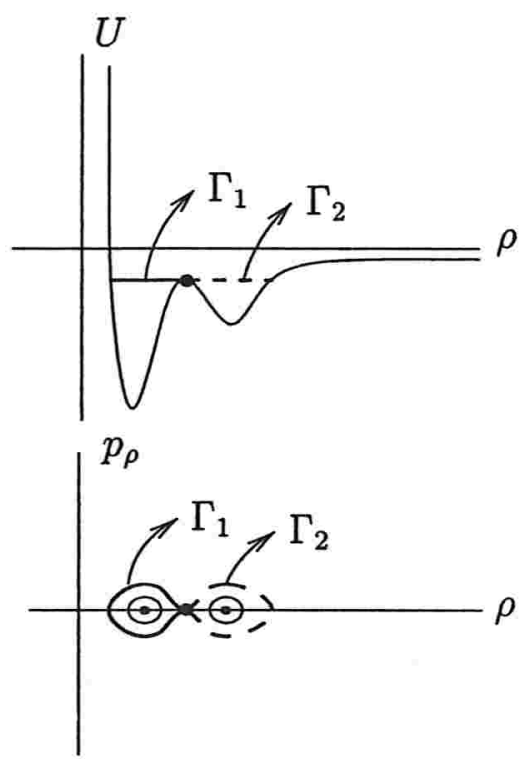

Figura 3.1: Exemplos de órbitas homoclínicas $\Gamma_{1}$ e $\Gamma_{2}$.

Poincaré. Uma primeira dificuldade vem do fato de que o equilíbrio contido no laço é instável. De maneira que, para ultrapassar esta dificuldade, escreve-se a aplicação de Poincaré para o laço sela-centro como uma composição de duas aplicações: uma relacionada à passagem das soluções próxima ao equilíbrio sela-centro e outra devida às excursões das soluções próximas à órbita homoclínica. Para definir tais aplicações, considera-se a parte quadrática de $\bar{H}(1.8)$, ou seja,

$$
\bar{H}_{Q}=\frac{1}{2}\left[p_{\rho}^{2}+p_{z}^{2}-|\nu|\left(\rho-\rho_{*}\right)^{2}+|\omega| z^{2}\right] .
$$

Segue-se, então, que $\left(\rho_{*}, 0\right)$ tem uma variedade unidimensional estável $W^{s}$, uma variedade unidimensional instável $W^{u}$ e uma variedade bidimensional central $W^{c}$. As aproximações lineares de tais variedades são dadas por:

$$
\begin{gathered}
W_{L}^{s}=\left\{\zeta=\left(\rho, z, p_{\rho}, p_{z}\right): p_{\rho}=-\sqrt{|\nu|}\left(\rho-\rho_{*}\right), p_{z}=z=0\right\} \\
W_{L}^{u}=\left\{\zeta=\left(\rho, z, p_{\rho}, p_{z}\right): p_{\rho}=\sqrt{|\nu|}\left(\rho-\rho_{*}\right), p_{z}=z=0\right\} \\
W_{L}^{c}=\left\{\zeta=\left(\rho, z, p_{\rho}, p_{z}\right): p_{\rho}=0, \rho=\rho_{*}\right\}
\end{gathered}
$$

Denota-se por $W_{+}^{s}\left(W_{+}^{u}\right)$ a parte de $W^{s}\left(W^{u}\right) \operatorname{com} \rho>\rho_{*}$ e por $W_{-}^{s}\left(W_{-}^{u}\right)$ a parte de $W^{s}\left(W^{u}\right)$ com $0<\rho<\rho_{*}$. Observa-se que a órbita homoclínica coincide ou com $W_{+}^{s}$ e $W_{+}^{u}$, ou com $W_{-}^{s}$ e $W_{-}^{u}$. A figura (3.2) esboça o primeiro caso. 


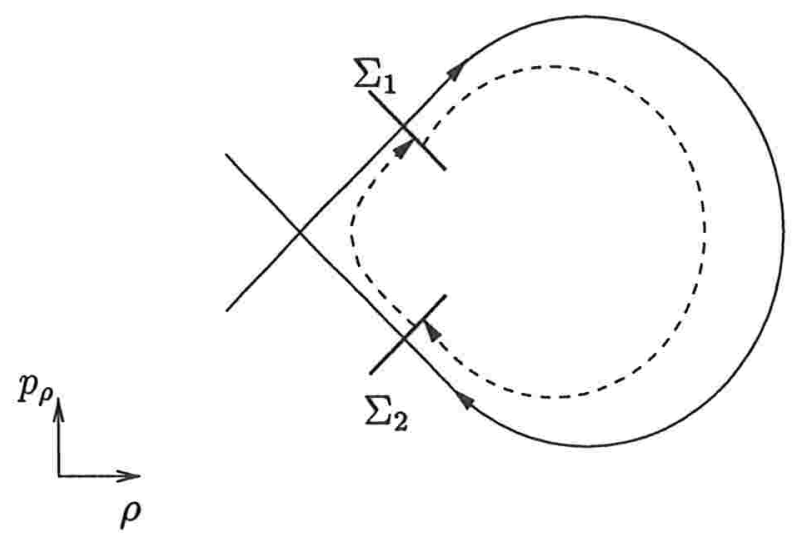

Figura 3.2: Seções de Poincaré $\Sigma_{1}$ e $\Sigma_{2}$, transversais às variedades $W_{+}^{s}$ e $W_{+}^{u}$, respectivamente.

Sejam $E_{0}$ a energia do equilíbrio sela-centro e $\Gamma_{E}$ as órbitas periódicas com energia $E<E_{0}$, as quais se acumulam na órbita homoclínica ao sela-centro, conforme Grotta Ragazzo (1997c) e Bastos de Figueiredo et al. (1998). Pode-se então, definir uma seção de Poincaré $\Sigma$ transversal ao laço sela-centro como

$$
\Sigma=\left\{\left(\rho=\rho_{0}, z, p_{\rho}<0, p_{z}\right\}\right.
$$

onde $\rho_{0}$ é uma constante (conveniente).

Usando a conservação da energia, considera-se a família a 1-parâmetro de aplicações de Poincaré, $f_{E}: \Sigma_{E} \rightarrow \Sigma_{E}$ onde $\Sigma_{E}$ denota a restrição de $\Sigma$ ao nível de energia $E$. Para cada $E$, toma-se $p_{\rho}=-\sqrt{2 E-p_{z}^{2}-z^{2}}$. Desta forma, tem-se que $\left(z, p_{z}\right)$ é um sistema de coordenadas para $\Sigma_{E}$, desde que $2 E-p_{z}^{2}-z^{2}>0$.

Após uma adequada parametrização local de tais seções(por $z, p_{z}$ e $E$ ), mostrase(ver Lerman (1991), Mielke et al. (1992)) que, para $\left|E-E_{0}\right|$ e $\|\xi\|:=\left\|\left(z, p_{z}\right)\right\|$ suficientemente pequenos, $\mathbf{f}_{E}$ pode ser aproximada pela família de aplicações(ver Grotta Ragazzo (1997b), Grotta Ragazzo (1997a))

$$
\mathbf{F}_{E}(\xi)=\mathbf{A R}\left[\bar{k}-\gamma \ln \left|\frac{1}{2} \sqrt{|\omega|}\right||\xi|^{2}-\left(E-E_{0}\right) \mid\right] \xi
$$

onde $\xi=\left(z, p_{z}\right) \in \mathbb{R}^{2},\|\xi\|>\sqrt{\left(\frac{2}{\sqrt{|\omega|}}\right)\left(E-E_{0}\right)}$ para $E>E_{0}, \mathbf{F}_{E_{0}}(\mathbf{0}):=0$ para 
$E=E_{0}, \bar{k}$ é uma constante(que pode ser removida por um reescalamento), $\gamma=\sqrt{\left|\frac{\omega}{\nu}\right|}$,

$$
\mathbf{A}=\left(\begin{array}{cc}
\alpha & 0 \\
0 & 1 / \alpha
\end{array}\right), \quad \alpha \geq 1
$$

e

$$
\mathbf{R}(\theta)=\left(\begin{array}{cc}
\cos \theta & -\operatorname{sen} \theta \\
\operatorname{sen} \theta & \cos \theta
\end{array}\right) .
$$

Logo, a dinâmica do laço sela-centro está relacionada aos invariantes $\alpha$ e $\gamma$. O parâmetro $\gamma$ é a razão entre os autovalores $\sqrt{|\omega|}$ e $\sqrt{|\nu|}$, e portanto é invariante sob mudança de variáveis. Já o parâmetro $\alpha$ é mais difícil de calcular e está relacionado à linearização do fluxo na órbita homoclínica.

\subsection{O Invariante $\alpha$ e sua Interpretação Geométrica}

Seguindo Grotta Ragazzo (1994) e Addas Zanata, Grotta Ragazzo (2001), denotase

$$
P(\rho)=2\left(E_{0}-U(\rho, 0)\right)
$$

e

$$
|\omega|+Q(\rho)=\frac{\partial^{2} U(\rho, 0)}{\partial z^{2}}
$$

Seja $\Gamma(t)$ a solução homoclínica à $\rho_{*}$ dada por:

$$
\begin{aligned}
\rho & =\Gamma(t) \\
p_{\rho} & =\dot{\Gamma}(t) \\
z & =p_{z}=0
\end{aligned}
$$

onde $\Gamma(t)$ satisfaz a equação

$$
\dot{x}^{2}=P(x)
$$

As componentes $\left(z, P_{z}\right)$ do campo de vetores linearizado em $\Gamma$ são

$$
\begin{aligned}
\dot{z} & =p_{z} \\
\dot{p}_{z} & =-[|\omega|+Q(\Gamma(t))] z .
\end{aligned}
$$


Segue que as equações acima são equivalentes à equação diferencial de segunda ordem em $z$ abaixo:

$$
\ddot{z}=-[|\omega|+Q(\Gamma(t))] z
$$

onde $Q(\Gamma(t))$ decresce exponencialmente para zero quando $t \rightarrow \pm \infty$. Esta equação tem uma solução complexa $\phi$ com o seguinte comportamento assintótico:

$$
\begin{array}{rll}
\phi(t) \rightarrow a \mathrm{e}^{i \sqrt{|\omega|} t}+b \mathrm{e}^{-i \sqrt{|\omega|} t} & \text { quando } & t \rightarrow-\infty \\
\phi(t) \rightarrow \mathrm{e}^{i \sqrt{|\omega|} t} & \text { quando } & t \rightarrow \infty
\end{array}
$$

$\operatorname{com} a, b \in \mathbb{C}$.

Em Grotta Ragazzo (1994), mostra-se que

$$
\alpha=|b|+\sqrt{|b|^{2}+1}
$$

onde $|b|$ é o parâmetro dado em (3.11).

Para uma interpretação geométrica de $\alpha$, observa-se que a variedade central do equilíbrio sela-centro é folheada por órbitas periódicas $\zeta_{\epsilon}$, uma para cada energia $E>E_{0}$. Em uma aproximação linear, $\zeta_{\epsilon}$ é um círculo no plano $\left\{\rho_{*}, 0, z, p_{z}\right\}$. A variedade estável de $\zeta_{\epsilon}$ é um cilindro que intercepta a seção $\Sigma_{E}$ em um círculo $C_{E}$. Por sua vez, $\mathbf{F}_{E}$ leva $C_{E}$ em uma curva fechada $C_{E}^{\prime}$ que é aproximadamente uma elipse, se $E-E_{0}$ é suficientemente pequeno, conforme a figura (3.3). Genericamente, $C_{E}$ intercepta $C_{E}^{\prime}$ transversalmente.

Considera-se, agora, $\mathcal{A}_{1}$ como sendo a área de $C_{E}$ e, $\mathcal{A}_{2}$ como sendo a área de $C_{E}^{\prime}$ exceto a parte desta área que pertence a $C_{E}$. Seja $\Delta$ a razão entre $\mathcal{A}_{2}$ e $\mathcal{A}_{1}$. Em (Addas Zanata, Grotta Ragazzo (2001)), é mostrado que

$$
\frac{\alpha+\alpha^{-1}}{2}=\frac{\operatorname{Tr}(\mathbf{A})}{2}=\frac{1}{\cos \left(\Delta \frac{\pi}{2}\right)}
$$

Portanto, o invariante $\alpha$ e seu inverso $\alpha^{-1}$ podem ser interpretados não só como os autovalores de $\mathbf{A}$ mas também como os semi-eixos de $C_{E}^{\prime}$. 


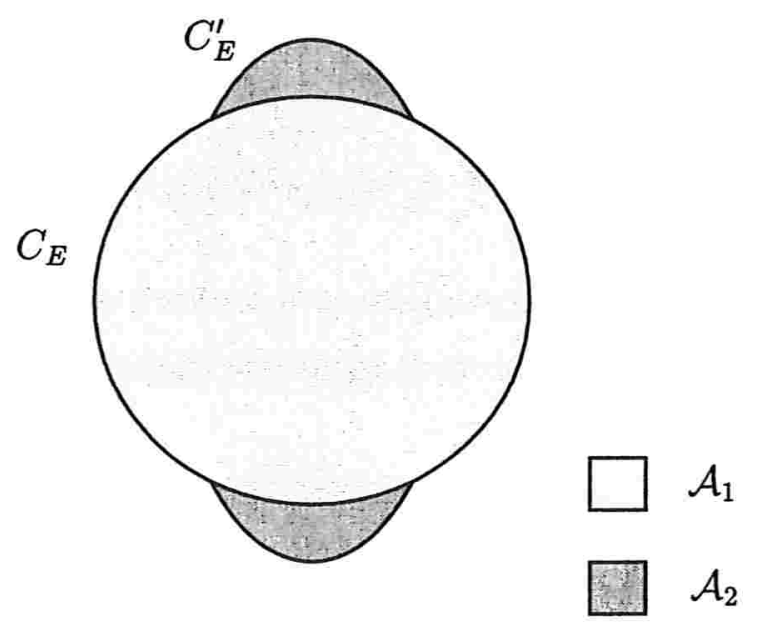

Figura 3.3: Comportamento da aplicação $\mathbf{F}_{E}$.

\subsection{Cálculo Aproximado do Invariante $\alpha$}

Ressalta-se que este cálculo foi feito numericamente e que a seguir dar-se-á a teoria pela qual foi desenvolvido o algoritmo. Pode-se ver no apêndice B o programa (3) usado para obter o invariante $\alpha$.

O invariante $\alpha$ é definido por (3.12), e pode ser interpretado geometricamente como na seção anterior. Para o cálculo numérico, obtém-se inicialmente a órbita homoclínica $\Gamma(t)$, integrando-se o sistema de equações

$$
\begin{aligned}
\dot{x} & =X \\
\dot{X} & =-U^{\prime}(x)
\end{aligned}
$$

com condições iniciais $x(0)=\rho_{\min }$ e $\dot{x}(0)=X(0)=0$.

Substitui-se a solução $\Gamma(t)$ em (3.9). Observa-se que, quando $t \rightarrow-\infty$, a equação (3.9) é equivalente à

$$
\begin{gathered}
\dot{z}=p_{z} \\
\dot{p_{z}}=-|\omega| z
\end{gathered}
$$


cujas curvas de nível são elipses.

No entanto, pretende-se partir de um círculo. Para tanto, toma-se uma mudança canônica de variáveis dada pela função geradora

$$
S=|\omega|^{1 / 4} z Y
$$

de onde, a equação (3.9) é transformada em

$$
\begin{aligned}
\dot{y} & =|\omega|^{1 / 2} Y \\
\dot{Y} & =-\left(|\omega|^{1 / 2}+|\omega|^{-1 / 2} Q(\Gamma(t))\right) y
\end{aligned}
$$

cujas curvas de nível são círculos, conforme desejado.

Neste momento, integra-se (3.15) de $-T$ à $T$ com condições iniciais $(1,0)$ e $(0,1)$. Desta forma, constrói-se uma matriz fundamental de soluções

$$
\mathbf{M}(t)=\left(\begin{array}{ll}
y_{1}(t) & y_{2}(t) \\
Y_{1}(t) & Y_{2}(t)
\end{array}\right)
$$

tal que $\mathbf{M}(-T)=\mathbf{I}$

Obtido $\mathbf{M}(T)$, como $\operatorname{det} \mathbf{M}(T)=1$, pode-se escrever sua representação polar

$$
\mathbf{M}(T)=\mathbf{S R}
$$

onde $\mathbf{S}$ é uma matriz simétrica e $\mathbf{R}$ é uma matriz de rotação.

Com isto, resulta que

$$
\mathbf{M}(T) \mathbf{M}(T)^{t}=\mathbf{S}^{2}
$$

Portanto, calculando-se os autovalores de $\mathbf{M}(T) \mathbf{M}(T)^{t}$, obtém-se os autovalores de $\mathbf{S}$ que são os semi-eixos da elipse, ou seja, o invariante $\alpha$ e seu inverso $\alpha^{-1}$.

Para obter M, observa-se que não é necessário integrar (3.15) de $-T$ à $T$ mas somente de 0 à $T$. Para confirmar esta observação, considera-se a função 


$$
g(t)=-\left(|\omega|^{1 / 2}+|\omega|^{-1 / 2} Q(\Gamma(t))\right)
$$

a qual é uma função par em $t$.

Seja

$$
\mathbf{N}(t):=\left(\begin{array}{cc}
z_{1}(t) & z_{2}(t) \\
Z_{1}(t) & Z_{2}(t)
\end{array}\right)
$$

a matriz fundamental de soluções com $\mathbf{N}(0)=\mathbf{I}$ obtida da integração a partir de $t=0$.

De fato, de $g(t)$ ser par, prova-se que $z_{1}(t)$ e $Z_{2}(t)$ são pares e $Z_{1}(t)$ e $z_{2}(t)$ são ímpares, donde resulta que

$$
\mathbf{N}(-T)=\left(\begin{array}{cc}
z_{1}(T) & -z_{2}(T) \\
-Z_{1}(T) & Z_{2}(T)
\end{array}\right)
$$

Desta forma,

$$
\mathbf{M}(t):=\mathbf{N}(t) \mathbf{N}(-T)^{-1}
$$

é uma matriz fundamental de soluções com $\mathbf{M}(-T)=\mathbf{I}$. Portanto, para obter $\mathbf{M}=$ $\mathbf{M}(T)$, basta calcular $\mathbf{N}(T)$.

\subsection{Estabilidade Orbital dos Laços Sela-Centro}

Neste momento, considera-se aplicação(3.2) restrita à energia $E_{0}$, ou seja, à energia do laço sela-centro. Após uma reescala conveniente de variáveis (Lerman (1991),Mielke et al. (1992),Grotta Ragazzo (1997a), Grotta Ragazzo (1997b)) tal aplicação pode ser reescrita como:

$$
\mathbf{F}(\xi)=\mathbf{A R}(\theta) \xi
$$

onde $\xi \in \mathbb{R}^{2}$, A e $\mathbf{R}$ são dados em (3.3) e (3.4) e $\theta=\theta(x)=-2 \gamma \ln |\xi|$.

Nota-se que F, é invariante por dilatações discretas, ou melhor, 


$$
\mathbf{F}\left(\mathrm{e}^{\frac{k \pi}{\gamma}} \xi\right)=\mathrm{e}^{\frac{k \pi}{\gamma}} \mathbf{F}(\xi)
$$

para todo $k \in \mathbb{Z}$.

Usando esta simetria, pode-se obter resultados acerca da estabilidade do ponto fixo $\xi_{*}=(0,0)=\mathbf{0}$.

De fato, se $\alpha=1$, a aplicação $\mathbf{F}$ é integrável e deixa invariantes todos os círculos centrados na origem. Logo, $\xi_{*}=\mathbf{0}$ é estável sob iterações de $\mathbf{F}$, para $\alpha=1$. Agora, longe de $\xi_{*}=\mathbf{0}, \mathbf{F}$ é analítica e do Teorema KAM (Kolmogorov, Arnold, Moser) prova-se que se $|\alpha-1|$ é suficientemente pequeno então existem curvas invariantes perto de $\xi_{*}$. Devido à simetria, têm-se que existem infinitas outras destas curvas se acumulando em 0 . Portanto, temos que $\xi_{*}=0$ também é estável se $\alpha$ está suficientemente próximo de 1(Grotta Ragazzo (1997c)).

Para $\alpha \gg 1$, usa-se novamente a simetria de dilatações e prova-se a instabilidade de $\xi_{*}$. Se $\mathbf{F}$ tem um ponto fixo $\bar{\xi}$ diferente de $\mathbf{0}$, então a aplicação possui uma família infinita de pontos fixos dados por (Grotta Ragazzo (1997a))

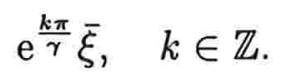

Mostra-se que, para $\alpha \neq 1$, a aplicação tem quatro famílias de pontos fixos, sendo que a primeira família é dada por $\xi_{k}, k \in \mathbb{Z}$, onde $\mathrm{e}^{\frac{k \pi}{\gamma}} \xi_{0} \mathrm{e}$

$$
\xi_{0}=\left(\left(\exp \left(\frac{\sigma}{\gamma}-\frac{\pi}{4 \gamma}\right) \cos \sigma, \exp \left(\frac{\sigma}{\gamma}-\frac{\pi}{4 \gamma}\right) \operatorname{sen} \sigma\right)\right.
$$

com

$$
\sigma=\operatorname{arctg}\left(\frac{1}{2}\right) \in\left(0, \frac{\pi}{4}\right)
$$

A segunda família de pontos fixos é dada por $-\xi_{k}$. Os autovalores $\left(\lambda, \lambda^{-1}\right)$ da linearização de $\mathbf{F}$ em torno de $\xi_{0} \mathrm{e}-\xi_{0}$ são determinados por

$$
\lambda+\lambda^{-1}=2+2 \gamma\left(\alpha-\alpha^{-1}\right)
$$

o que implica que todos os pontos fixos $\pm \xi_{k}$ são hiperbólicos.

Admitindo-se que a variedade instável de $\xi_{0}$ intercepta transversamente a variedade estável de $\xi_{1}$, da simetria por dilatações de $\mathbf{F}$, segue que a variedade instável de 


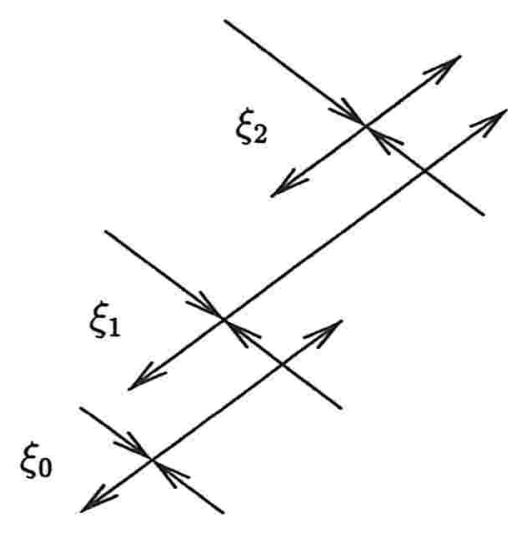

Figura 3.4: Cadeia heteroclínica transversal.

$\xi_{k}$ intercepta transversalmente a variedade estável de $\xi_{k+1}$,conforme ilustrado em 3.4. Logo, a existência de uma órbita heteroclínica transversal conectando $\xi_{0}$ a $\xi_{1}$ implica na existência de duas cadeias heteroclínicas infinitas, ou "cadeias de transição de Arnold", portanto, tem-se a instabilidade de $\xi=0$. Foi provado em Grotta Ragazzo (1997b) a existência de tal cadeia de transição para $\gamma\left(\alpha-\alpha^{-1}\right)>1$.

Além disso, em Grotta Ragazzo (1997c) também foi obtido numericamente uma curva $Z$ tal que se $1 \leq \alpha<Z(\gamma)$ então $\xi_{*}$ é estável; de outro modo $\xi_{*}$ é instável. Tal curva $Z$ é aproximadamente dada por

$$
\gamma\left(\alpha-\alpha_{-1}\right)=\frac{1}{\sqrt{2}}
$$

Alguns valores de $\alpha$ foram calculados e usando o critério acima, a estabilidade das órbitas homoclínicas correspondentes foram classificadas, conforme pode-se ver na tabela a seguir.

Observa-se que $c=\gamma\left(\alpha-\alpha_{-1}\right)$ e que para cada par $(L, \Lambda)$ com dois valores de $\alpha$, os casos $(\star)$ correspondem aos primeiros poços(mais próximos da origem) e os casos $(\star \star)$ correspondem aos segundos poços. 


\begin{tabular}{|c|c|c|c|c|c|}
\hline$L$ & $\Lambda$ & $\gamma$ & $\alpha$ & $c$ & Estabilidade \\
\hline 4.0 & 6.0 & 1.163755 & 8.241042 & 13.10320 & instável $(\star)$ \\
\hline 4.0 & 6.0 & 1.613755 & 3.148750 & 4.568805 & instável $(\star \star)$ \\
\hline 4.0 & 7.0 & 2.549510 & 8.466394 & 1.000581 & instável $(\star)$ \\
\hline 4.0 & 7.0 & 2.549510 & 21.28402 & 0.002960558 & estável( $(\star \star)$ \\
\hline 5.0 & 11.0 & 1.702274 & 8.026729 & 13.45162 & instável( $(\star)$ \\
\hline 5.0 & 11.0 & 1.702274 & 1.223218 & 0.6906163 & 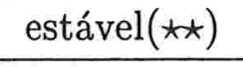 \\
\hline 10.0 & 80.0 & 1.722602 & 7.782857 & 13.18543 & instável $(\star)$ \\
\hline 10.0 & 80.0 & 1.722602 & 1.021520 & 0.07253288 & estável( $\star \star)$ \\
\hline 4.0 & 5.740740740 & 1.544479 & 8.061992 & 12.260006 & instável \\
\hline 5.0 & 10.25925926 & 1.588291 & 7.844429 & 12.256760 & instável \\
\hline 3.0 & 3.0 & 1.414214 & 9.039812 & 12.627784 & instável \\
\hline 4.0 & 3.0 & 1.245348 & 7.301817 & 8.922747 & instável \\
\hline 4.0 & 4.0 & 1.310052 & 7.657177 & 9.860210 & instável \\
\hline 5.0 & 3.0 & 1.229856 & 6.613553 & 7.947757 & instável \\
\hline 4.0 & 1.0 & 1.172604 & 6.350896 & 7.262449 & instável \\
\hline 3.0 & 1.0 & 1.097134 & 7.112312 & 7.648903 & instável \\
\hline 5.0 & 1.0 & 1.198332 & 6.086740 & 7.097062 & instável \\
\hline 4.0 & -2.0 & 1.154754 & 4.246315 & 4.631505 & instável \\
\hline 4.0 & 0.0 & 1.154736 & 5.763194 & 6.454602 & instável \\
\hline 4.0 & 0.0 & 1.187342 & 5.808461 & 6.692213 & instável \\
\hline
\end{tabular}

\subsection{Atratores para a Aplicação Discreta no Toro}

Neste momento, considera-se o caso em que o laço é instável, ou seja, quando $\gamma\left(\alpha-\alpha^{-1}\right)>1$. E observa-se o comportamento da aplicação discreta, porém em um outro espaço, descrito a seguir.

Usando a propriedade (3.17) da simetria das dilatações discretas, define-se a seguinte relação de equivalência $\sim$, em $\mathbb{R}^{2} \backslash\{0\}$, por

$$
x \sim y \quad \Leftrightarrow \quad x=\mathrm{e}^{\frac{k \pi}{\gamma}}, \quad k \in \mathbb{Z},
$$




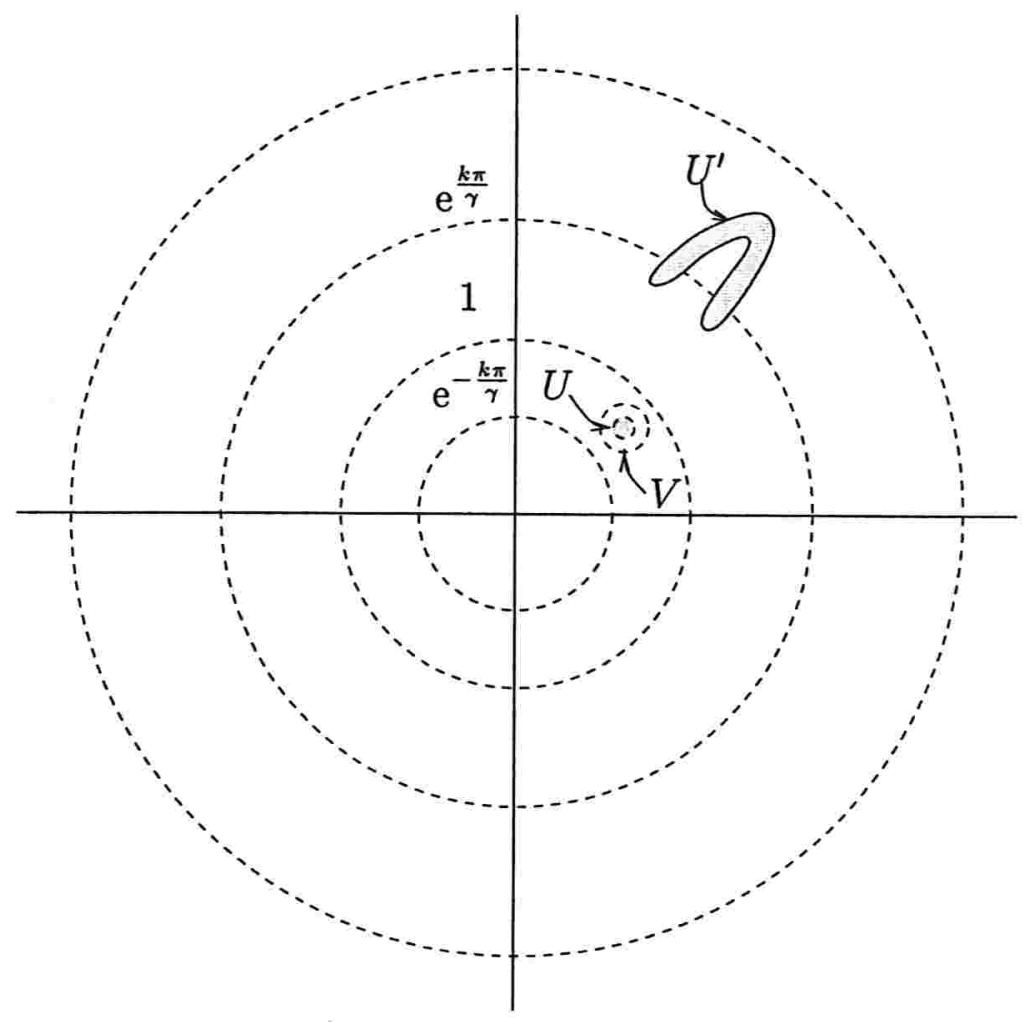

Figura 3.5: Não-preservação da área da aplicação $\hat{\mathbf{F}}_{E_{0}}$.

e toma-se

$$
\mathrm{T}^{2}=\mathbb{R} \backslash\{0\} / \sim
$$

com a projeção canônica $\pi: \mathbb{R}^{2} \backslash\{\mathbf{0}\} \rightarrow \mathrm{T}^{2}$.

Com isto, pode-se definir a aplicação

$$
\hat{\mathbf{F}}: \mathrm{T}^{2} \rightarrow \mathrm{T}^{2}
$$

por

$$
\pi \circ \mathbf{F}=\hat{\mathbf{F}} \circ \pi
$$

Observa-se que $\hat{\mathbf{F}}$ não preserva área(ver figura 3.5). Mais ainda, em Addas Zanata, Grotta Ragazzo (2002), mostra-se a existência de atratores periódicos para a aplicação $\hat{\mathbf{F}}$, para alguns pares $(\gamma, \alpha)$. Tais atratores correspondem à órbitas de $\mathbf{F}$ que escapam com uma velocidade exponencial da origem. 


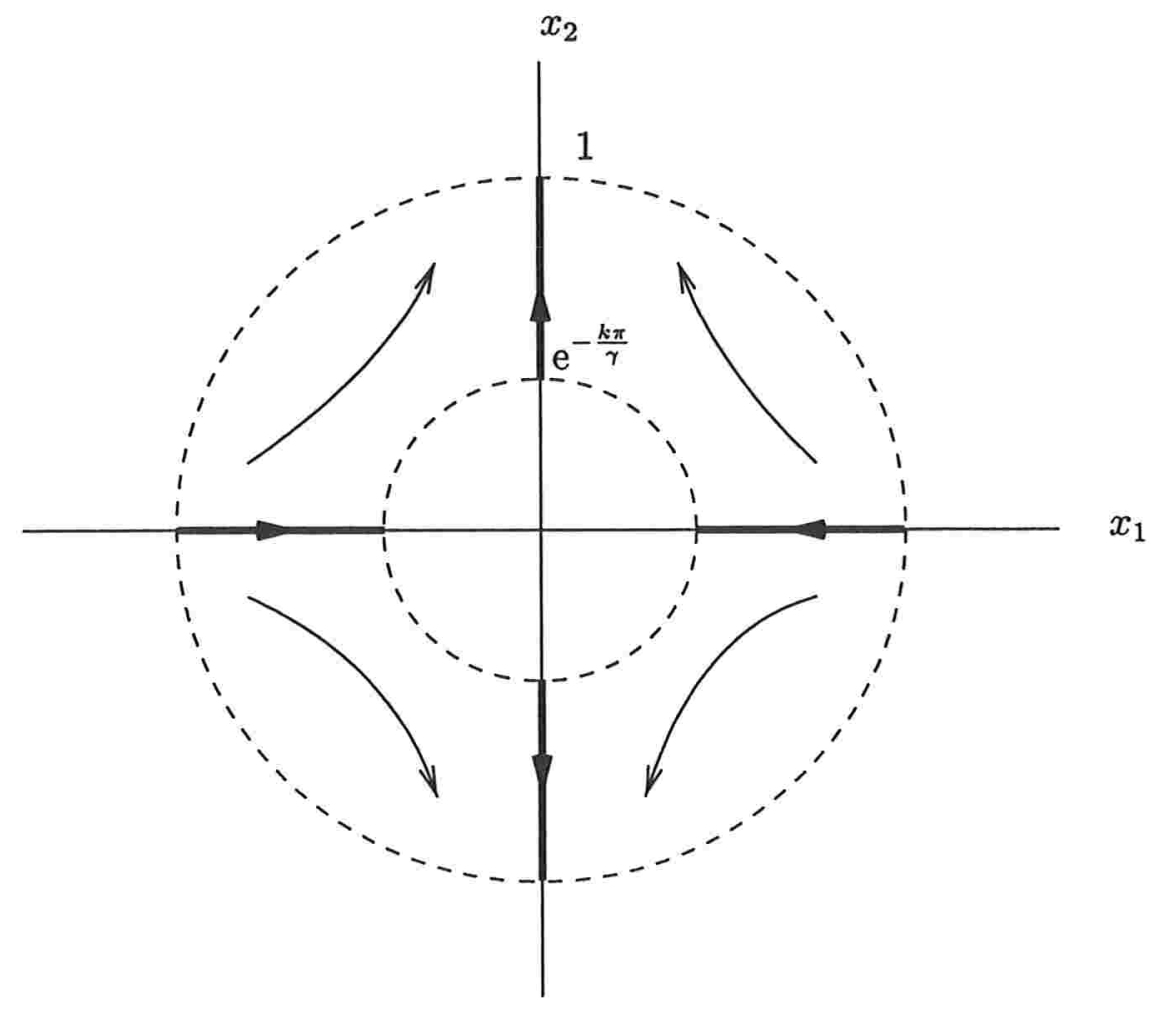

Figura 3.6: Círculos invariantes da aplicação $\hat{\mathbf{G}}$ induzida da aplicação hiperbólica G dada por (3.20).

Para ilustrar o comportamento de tais atratores, o exemplo seguinte é considerado. Seja $\mathbf{G}$ a aplicação hiperbólica dada por

$$
\mathbf{G}\left(x_{1}, x_{2}\right)=\left(2^{-1} x_{1}, 2 x_{2}\right)
$$

Como $\Phi(x)=\mathrm{e}^{\frac{k \pi}{\gamma}} x$ comuta com qualquer aplicação linear, a aplicação $\mathbf{G}$ induz uma aplicação

$$
\hat{\mathbf{G}}: \mathrm{T}^{2} \rightarrow \mathrm{T}^{2}
$$

por

$$
\pi \circ \mathbf{G}=\hat{\mathbf{G}} \circ \pi
$$

Da figura 3.6, têm-se que $\hat{\mathrm{G}}$ é dissipativa e tem 4 círculos invariantes, onde os 2 círculos relativos ao eixo $x_{1}$ são repulsores e os 2 círculos relativos ao eixo $x_{2}$ são atratores. Além disso, ao iterar $\mathrm{G}$ sobre uma vizinhança pequena de condições iniciais próxima à origem, resulta que tais iteradas se aglomeram em torno do eixo $x_{2}$. 
Verifica-se, de fato, que tais atratores existem para este caso como pode ser visto nos casos ilustrados a seguir. Primeiramente, foram obtidas as seções de Poincaré e discreta para o laço sela-centro. Em seguida, foi feito uma reescalamento do plano para o anel, onde observou-se a presença de um atrator de ordem 2. Para que o atrator ficasse mais vísivel, desprezou-se as primeiras iteradas, o que é visto nas figuras (3.11) e (A.1). O objetivo da obtenção das duas seções, a de Poincaré e a discreta é comprovar, mais uma vez, que a aplicação discreta é uma boa aproximação da seção de Poincaré.

Este trabalho limitou-se a apresentar a existência de exemplos de atratores. No entanto, fica em aberto um estudo do significado deles para os anéis de Júpiter.

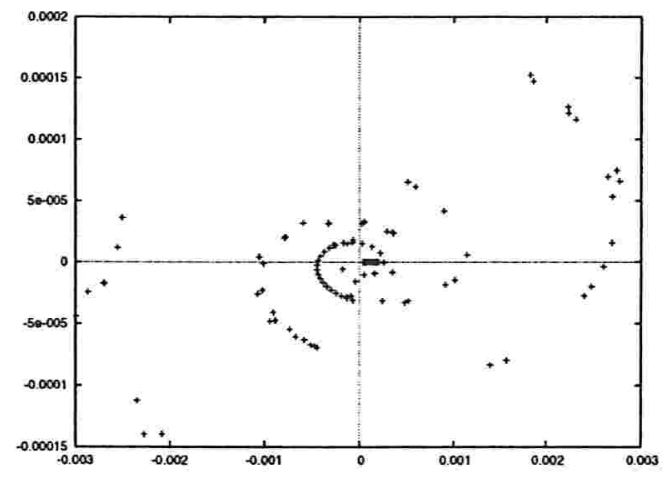

Figura 3.7: Seção de Poincaré onde $L=4, \Lambda=6$ e $\alpha=3,14$, obtida pelo programa (4) do apêndice B.

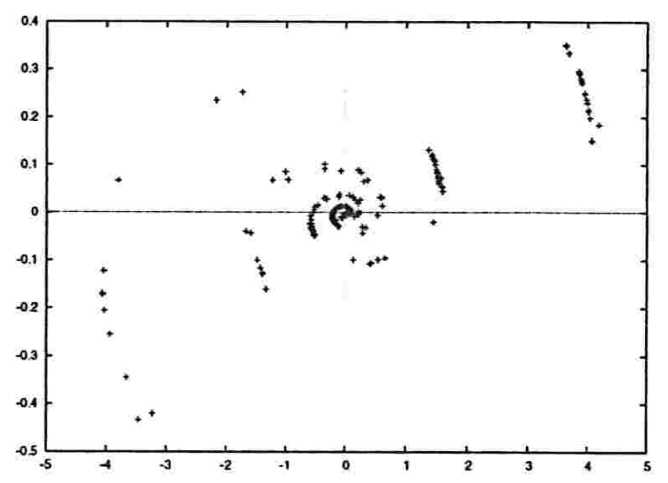

Figura 3.8: Seção discreta onde $L=4$, $\Lambda=6$ e $\alpha=3,14$, obtida pelo programa (5) do apêndice $\mathrm{B}$. 


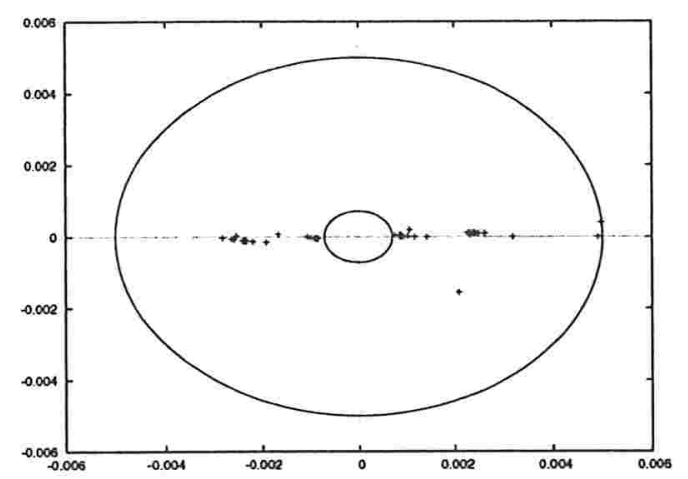

Figura 3.9: Seção (3.7) reescalada para 0 anel onde $L=4, \Lambda=6 \mathrm{e}$ $\alpha=3,14$, obtida pelo programa (6) do apêndice B.

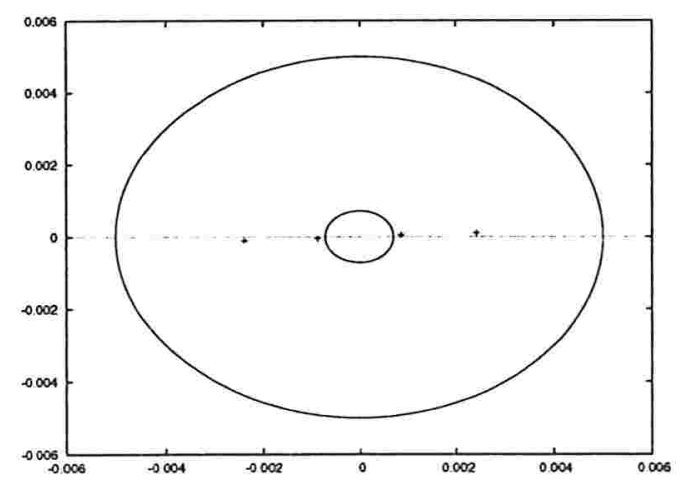

Figura 3.11: Atrator da seção (3.9), obtido pelo programa (6) do apêndice B.

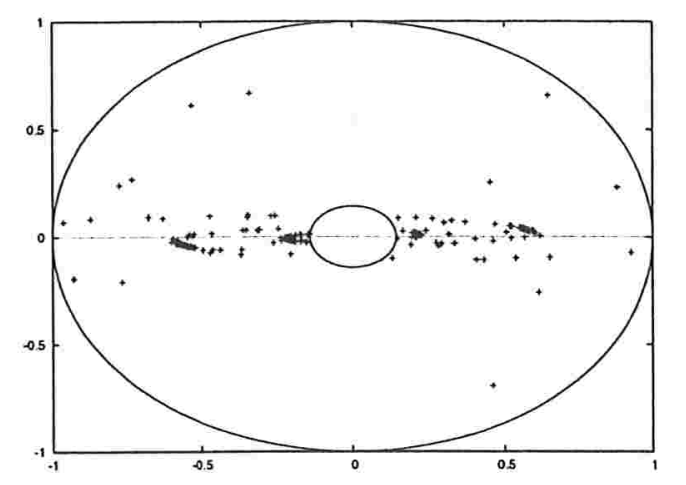

Figura 3.10: Seção (3.8) reescalada para o anel onde $L=4, \Lambda=6 \mathrm{e}$ $\alpha=3,14$, obtida pelo programa (7) do apêndice $B$.

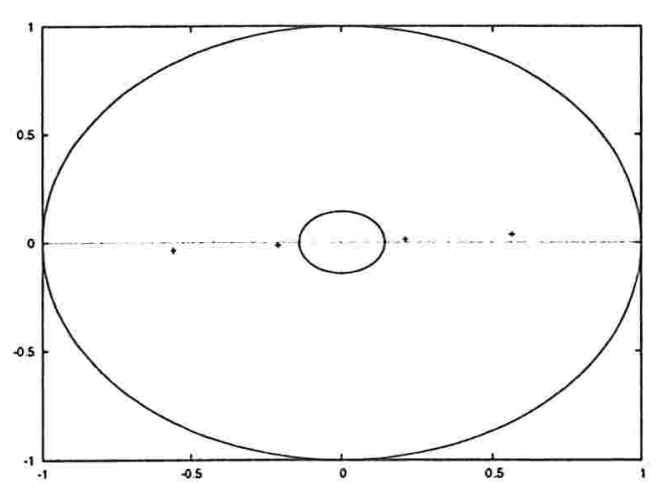

Figura 3.12: Atrator da seção (3.10), obtido pelo programa (7) do apêndice B. 
ApÊNDICE A

\section{Dados Planetários de Júpiter e}

\section{Saturno}

1. Anéis de Júpiter

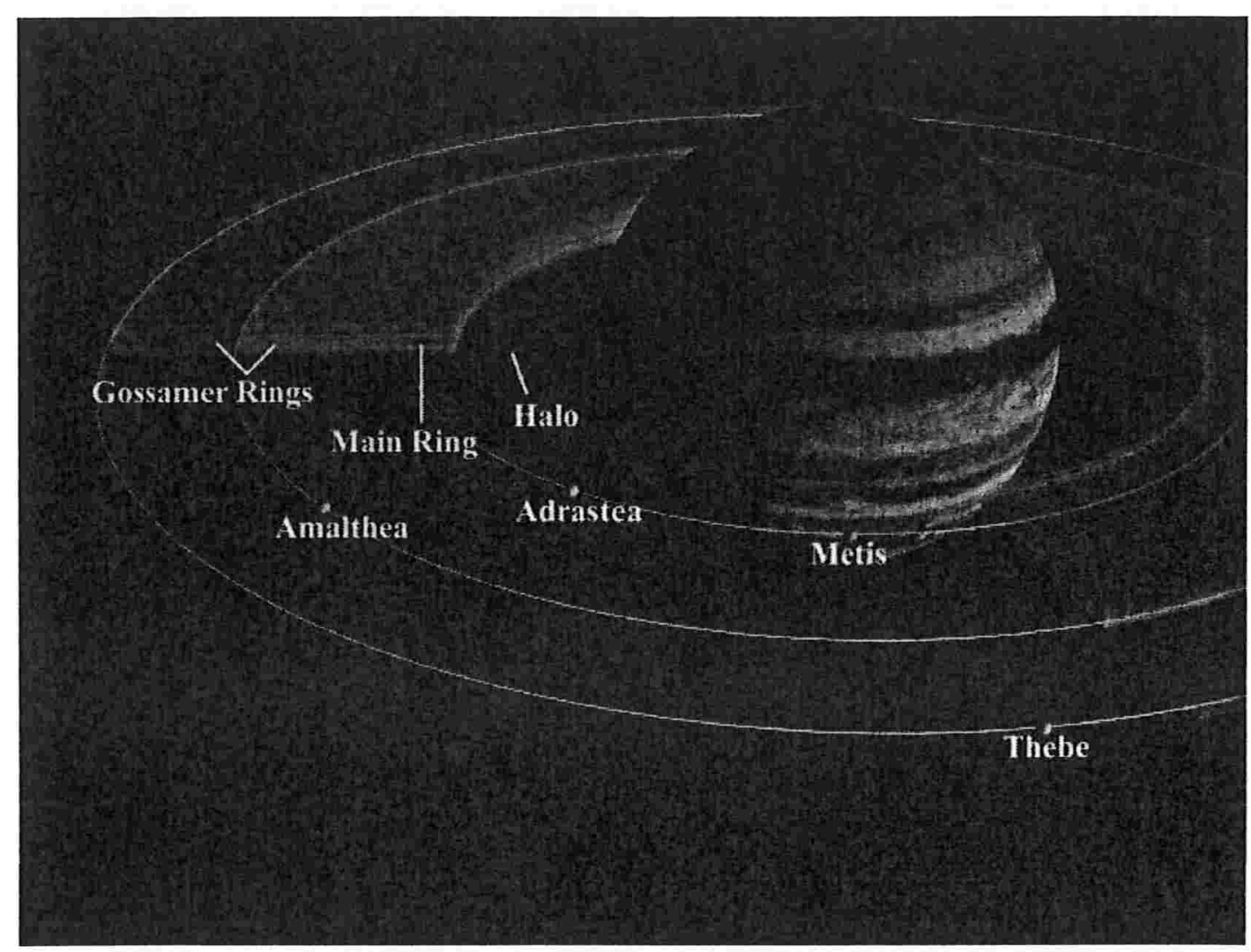

Figura A.1: Anéis de Júpiter e seus principais satélites. 
2. Comparações dos dados de Júpiter com os dados da Terra

\begin{tabular}{|l|c|c|c|}
\hline Dados & Júpiter & Terra & Razão(Júpiter/Terra) \\
\hline \hline Massa $\left(10^{24} \mathrm{~kg}\right)$ & $1.898,6$ & 5,9736 & 317,83 \\
\hline Volume $\left(10^{10} \mathrm{~km}^{3}\right)$ & 143.128 & 108,321 & 1321,33 \\
\hline Raio $(\mathrm{km})$ Equatorial & 71.492 & $6.378,1$ & 11,209 \\
\hline Polar & 66.854 & $6.356,8$ & 10,517 \\
\hline Raio Médio Volumétrico km & 69.911 & 6.371 & 10,973 \\
\hline Elipticidade & 0,06487 & 0,00335 & 19,36 \\
\hline Densidade Média $\left(\mathrm{kg} / \mathrm{m}^{3}\right)$ & 1.326 & 5.515 & 0,240 \\
\hline Gravidade $\left(\mathrm{m} / \mathrm{s}^{2}\right)$ & 24,79 & 9,80 & 2,530 \\
\hline Aceleração $\left(\mathrm{m} / \mathrm{s}^{2}\right)$ & 23,12 & 9,78 & 2,364 \\
\hline Velocidade de Escape $(\mathrm{km} / \mathrm{s})$ & 59,5 & 11,19 & 5,32 \\
\hline GM $\left(\times 10^{6} \mathrm{~km}{ }^{3} / \mathrm{s}^{2}\right)$ & 126,686 & 0,3986 & 317,81 \\
\hline Momento de Inércia $\left(\mathrm{I} / \mathrm{MR}^{2}\right)$ & 0,254 & 0,3308 & 0,768 \\
\hline $\mathrm{J}_{2}\left(\times 10^{-6}\right)$ & 14.736 & $1.082,63$ & 13,611 \\
\hline Número de Satélites Naturais & 63 & 1 & \\
\hline
\end{tabular}

3. Comparações dos dados de Saturno com os dados da Terra

\begin{tabular}{|l|c|c|c|}
\hline Dados & Saturno & Terra & Razão(Saturno/Terra) \\
\hline \hline Massa $\left(10^{24} \mathrm{~kg}\right)$ & 568,46 & 5,9736 & 95,159 \\
\hline Volume $\left(10^{10} \mathrm{~km}^{3}\right)$ & 82.713 & 108,321 & 763,59 \\
\hline Raio $(\mathrm{km})$ Equatorial & 60.268 & $6.378,1$ & 9,449 \\
\hline Polar & 54.364 & $6.356,8$ & 8,552 \\
\hline Raio Médio Volumétrico km & 58.232 & 6.371 & 9,140 \\
\hline Elipticidade & 0,09796 & 0,00335 & 29,24 \\
\hline Densidade Média $\left(\mathrm{kg} / \mathrm{m}^{3}\right)$ & 687 & 5.515 & 0,125 \\
\hline Gravidade $\left(\mathrm{m} / \mathrm{s}^{2}\right)$ & 10,44 & 9,80 & 1,065 \\
\hline Aceleração $\left(\mathrm{m} / \mathrm{s}^{2}\right)$ & 8,96 & 9,78 & 0,916 \\
\hline Velocidade de Escape $\left(\mathrm{km}^{2} \mathrm{~s}\right)$ & 35,5 & 11,19 & 3,172 \\
\hline GM $\left(\times 10^{6} \mathrm{~km}{ }^{3} / \mathrm{s}^{2}\right)$ & 37,931 & 0,3936 & 95,16 \\
\hline Momento de Inércia $\left(\mathrm{I} / \mathrm{MR}^{2}\right)$ & 0,210 & 0,3308 & 0,635 \\
\hline $\mathrm{J}_{2}\left(\times 10^{-6}\right)$ & 16.298 & $1.082,63$ & 15,504 \\
\hline Número de Satélites Naturais & 33 & 1 & \\
\hline
\end{tabular}


4. Parâmetros Orbitais de Júpiter

\begin{tabular}{|l|c|c|c|}
\hline Dados & Júpiter & Terra & Razão(Júpiter/Terra) \\
\hline \hline Semi-eixo Maior $\left(10^{6} \mathrm{~km}\right)$ & 778,57 & 149,60 & 5,204 \\
\hline Período da Órbita Sideral (dias) & $4.332,589$ & 365.256 & 11,862 \\
\hline Período da Órbita Tropical (dias) & $4.330,595$ & 365,242 & 11,857 \\
\hline Periélio $\left(10^{6} \mathrm{~km}\right)$ & 740,52 & 147,09 & 5,034 \\
\hline Afélio $\left(10^{6} \mathrm{~km}\right)$ & 816,62 & 152,10 & 5,369 \\
\hline Período Sinódico (dias) & 398,88 & - & - \\
\hline Velocidade Média Orbital (km/s) & 13,07 & 29,78 & 0,439 \\
\hline Velocidade Orbital Máxima (km/s) & 13,72 & 30,29 & 0,453 \\
\hline Velocidade Orbital Mínima (km/s) & 12,44 & 29,29 & 0,425 \\
\hline Inclinação da Órbita (graus) & 1,304 & 0,00 & - \\
\hline Excentricidade da Órbita & 0,0489 & 0,0167 & 2,928 \\
\hline Período de Rotação Sideral (horas) & 9,9250 & 23,9345 & 0,415 \\
\hline Comprimento dos dias (horas) & 9,9254 & 24,0 & 0,414 \\
\hline Obliquidade da Órbita (graus) & 3,13 & 23,45 & 0,133 \\
\hline
\end{tabular}

5. Parâmetros Orbitais de Saturno

\begin{tabular}{|l|c|c|c|}
\hline Dados & Saturno & Terra & Razão(Saturno/Terra) \\
\hline \hline Semi-eixo Maior $\left(10^{6} \mathrm{~km}\right)$ & $1.433,53$ & 149,60 & 9,582 \\
\hline Período da Órbita Sideral (dias) & $10.759,22$ & 365,256 & 29,457 \\
\hline Período da Órbita Tropical (dias) & $10.746,94$ & 365,242 & 29,424 \\
\hline Periélio $\left(10^{6} \mathrm{~km}\right)$ & $1.352,53$ & 147,09 & 9,195 \\
\hline Afélio $\left(10^{6} \mathrm{~km}\right)$ & $1.514,50$ & 152,10 & 9,957 \\
\hline Período Sinódico (dias) & 378,09 & - & - \\
\hline Velocidade Média Orbital (km/s) & 9,69 & 29,78 & 0,325 \\
\hline Velocidade Orbital Máxima (km/s) & 10,18 & 30,29 & 0,336 \\
\hline Velocidade Orbital Mínima (km/s) & 9,09 & 29,29 & 0,310 \\
\hline Inclinação da Órbita (graus) & 2,485 & 0,00 & - \\
\hline Excentricidade da Órbita & 0,0565 & 0,0167 & 3,383 \\
\hline Período de Rotação Sideral (horas) & 10,656 & 23,9345 & 0,445 \\
\hline Comprimento dos dias (horas) & 10,656 & 24,0 & 0,444 \\
\hline Obliquidade da Órbita (graus) & 26,73 & 23,45 & 1,140 \\
\hline
\end{tabular}


6. Elementos Órbitais Médios (J2000)

\begin{tabular}{|l|c|c|}
\hline Dados & Júpiter & Saturno \\
\hline \hline Semi-eixo Maior (AU) & 5,20336301 & 9,53707032 \\
\hline Excentricidade Orbital & 0,04839266 & 0,05415060 \\
\hline Inclinação Orbital (graus) & 1,30530 & 2,48446 \\
\hline Longitude do Nodo Ascendente (graus) & 100,55615 & 113,71504 \\
\hline Longitude do Periélio (graus) & 14,75385 & 92,43194 \\
\hline Longitude Média (graus) & 34,40438 & 49,94432 \\
\hline
\end{tabular}

7. Magnetosfera de Júpiter

\begin{tabular}{|c|c|}
\hline Dados & Júpiter \\
\hline Campo de Dipolo Efetivo & $4,28\left(\right.$ gauss $\left.\times \mathrm{Rj}^{3}\right)$ \\
\hline Desvio do Dipolo em Relação ao Eixo de Rotação (graus) & 9,6 \\
\hline Longitude do Desvio (graus) & 201,7 \\
\hline Distância Offset (do Centro do Planeta ao Centro do Dipolo) & $0,131 \mathrm{Rj}$ \\
\hline Latitude/Longitude do Vetor Offset(graus) & $-8,0 / 148,57$ \\
\hline
\end{tabular}

Nota: $\mathrm{Rj}$ denota o raio Jovian $=71,398 \mathrm{~km}$.

\section{Magnetosfera de Saturno}

\begin{tabular}{|l|c}
\hline Dados & Saturno \\
\hline \hline Campo de Dipolo Efetivo & 0,210 (gauss $\times \mathrm{Rs}^{3}$ ) \\
\hline Desvio do Dipolo em Relação ao Eixo de Rotação (graus) & $<1$ \\
\hline Longitude do Desvio (graus) & - \\
\hline Distância Offset (do Centro do Planeta ao Centro do Dipolo) & $0,04-0,05$ Rs(direção norte) \\
\hline
\end{tabular}

Nota: Rs denota o raio Saturniano $=60,330 \mathrm{~km}$.

9. Dados dos Anéis de Júpiter e Saturno 


\begin{tabular}{|l|c|c|}
\hline Anel & Raio $(\mathrm{km})$ & Raio Equatorial \\
\hline \hline Equador de Júpiter & 71.492 & 1,000 \\
\hline Halo & $100.000-122.000$ & $1,40-1,71$ \\
\hline Principal & $122.000-129.000$ & $1,71-1,81$ \\
\hline Gossamer (interno) & $129.200-182.000$ & $1,81-2,55$ \\
\hline Gossamer (externo) & $182.000-224.900$ & $2,55-3,15$ \\
\hline \hline Equador de Saturno & 60.268 & 1,000 \\
\hline F (centro do anel) & 140.180 & 2,326 \\
\hline G (parte interna) & 170.000 & 2,82 \\
\hline E (parte interna) & 181.000 & 3 \\
\hline
\end{tabular}

Todos os dados aqui apresentados foram obtidos no site da "National Space Science Data Center". 



\section{Programas numéricos}

\section{Programa para o cálculo da curva $\chi$}

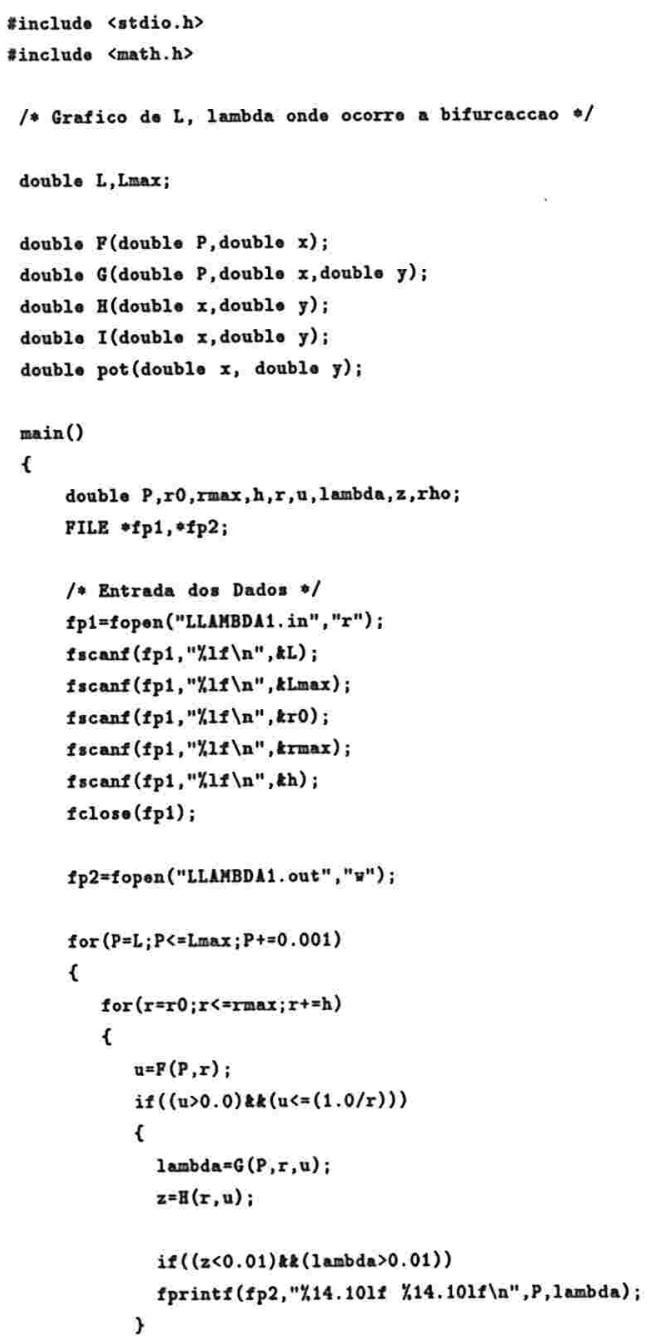




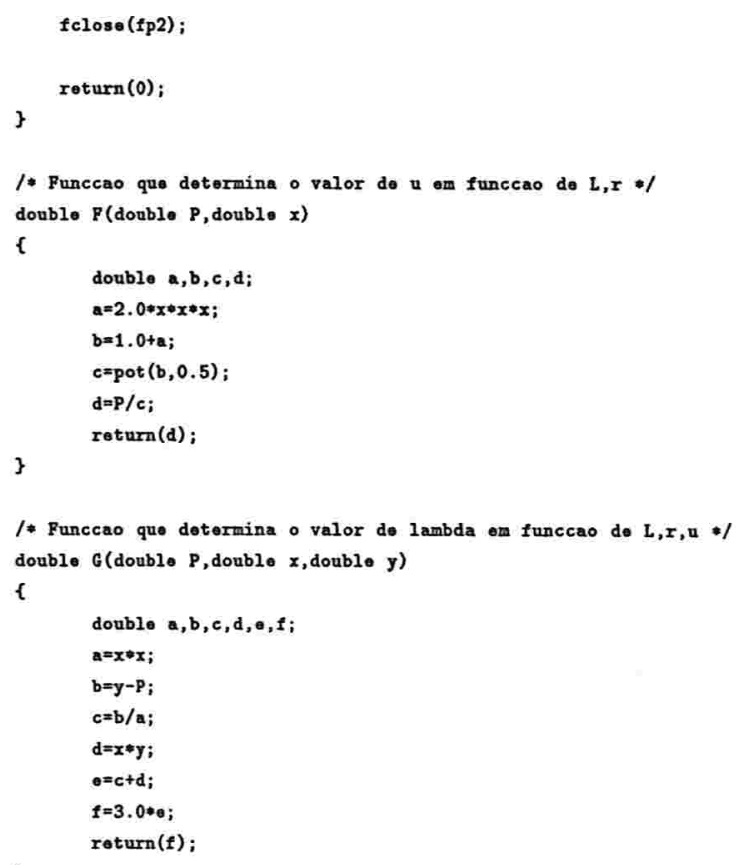

2. Programa para a verificação da região de Hill através do potencial U em função de $(\rho, z)$

\#includo 〈stdio.h〉 


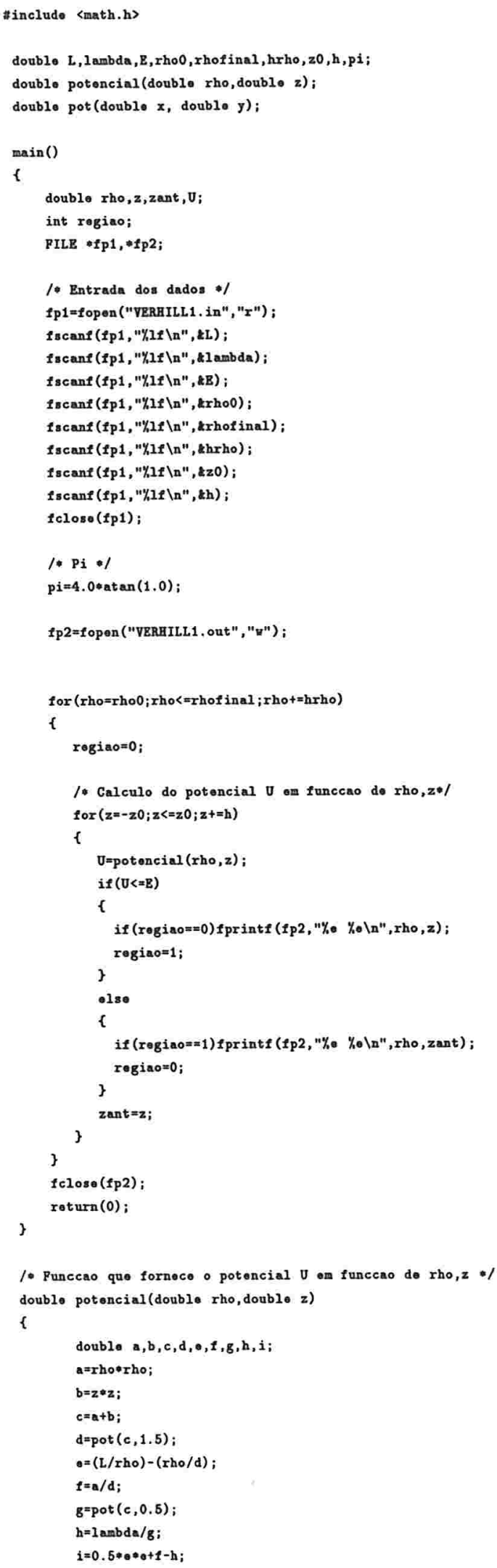


\}

roturn(i);

1. Funccao que fornece a potencia y de um numero real $x *$ /

double pot (double $x$, double $y$ )

t

if $(x>0.0)$

return $(\exp (y+\log (x)))$.

return(0.0);

\}

3. Programa para o cálculo do invariante $\alpha$ que, junto com $\gamma$, fornece o valor $c$ com o qual temos um critério de estabilidade para o laço sela-centro

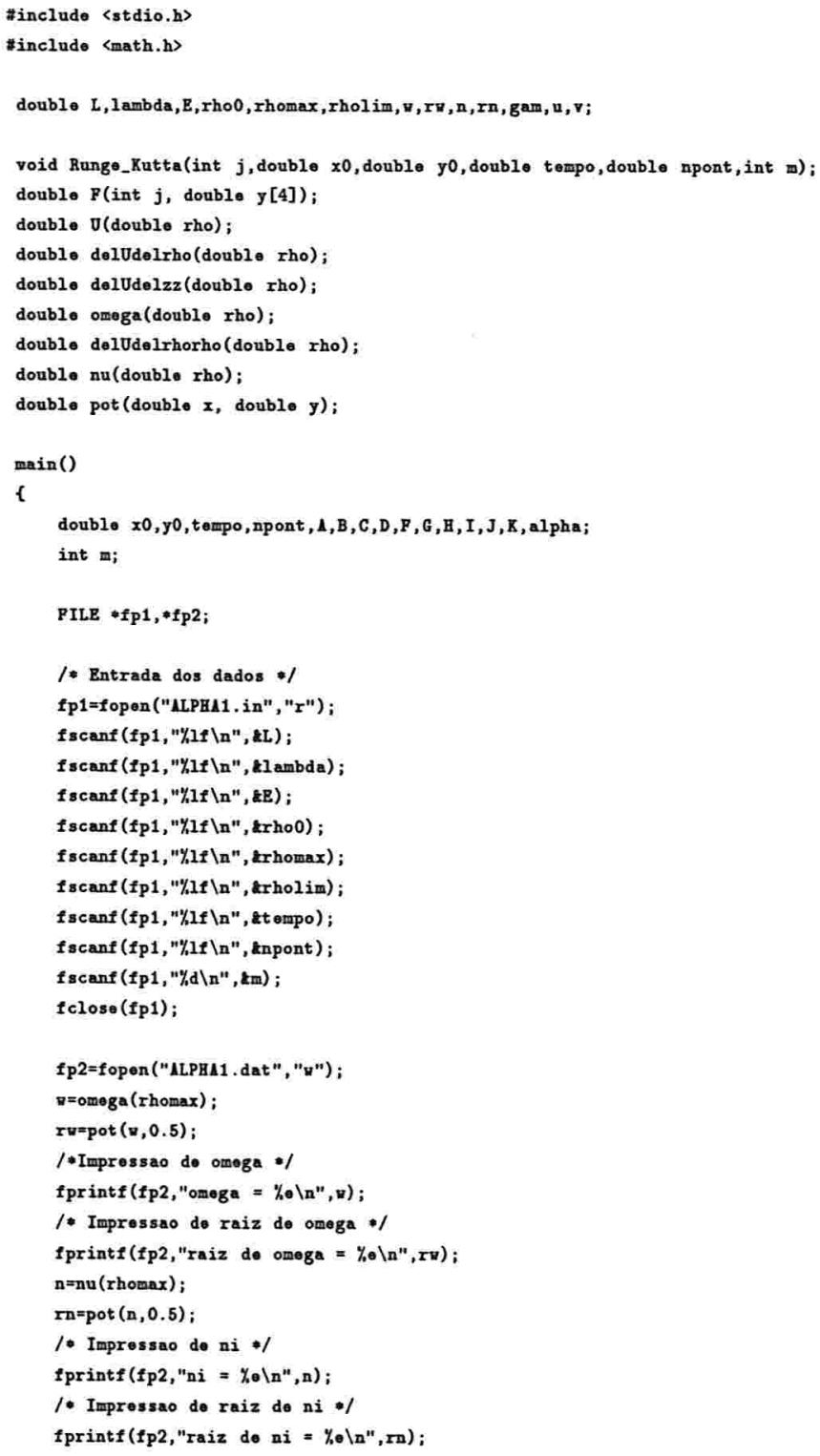




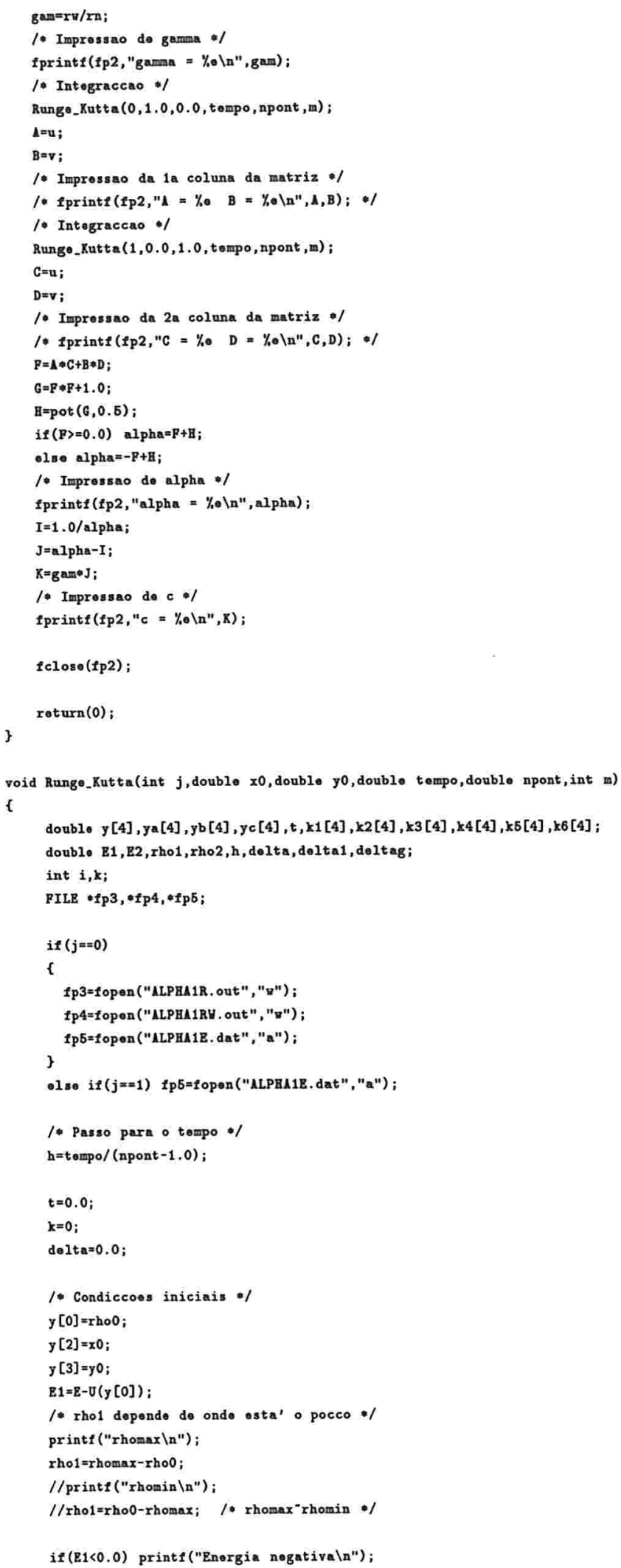




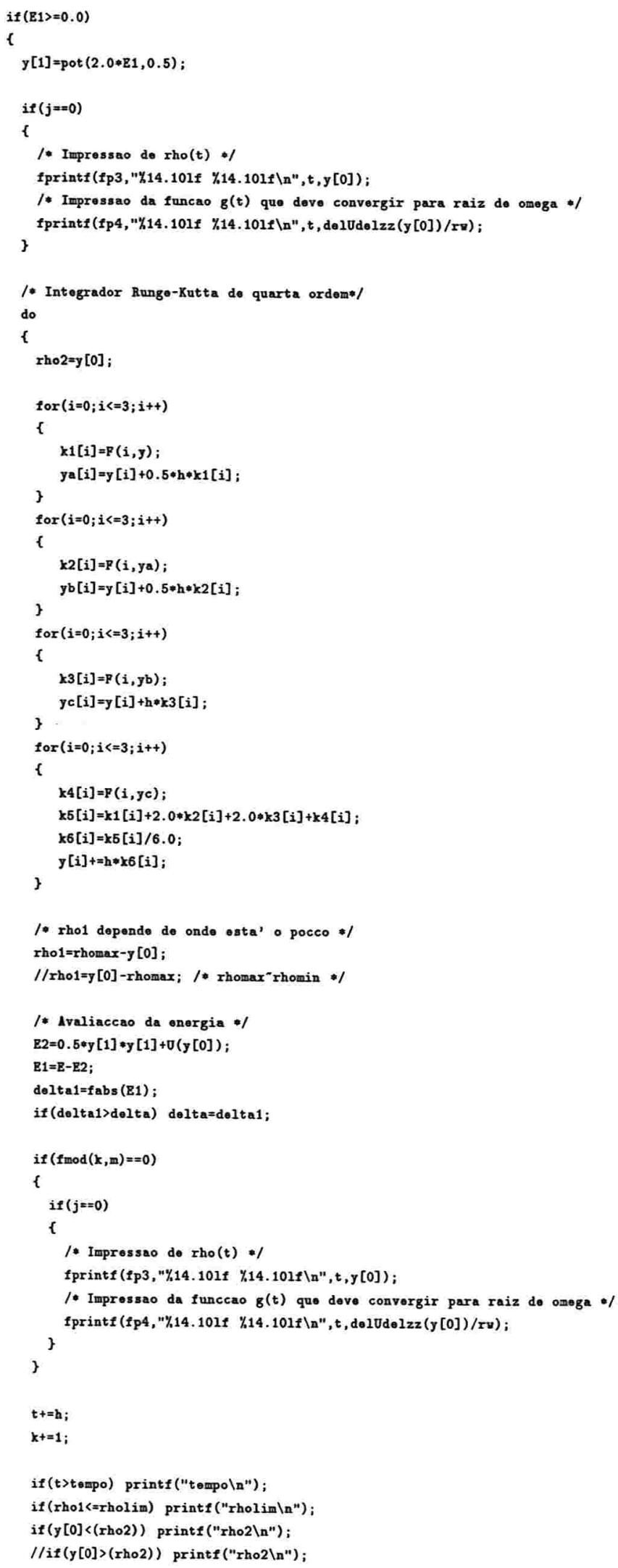




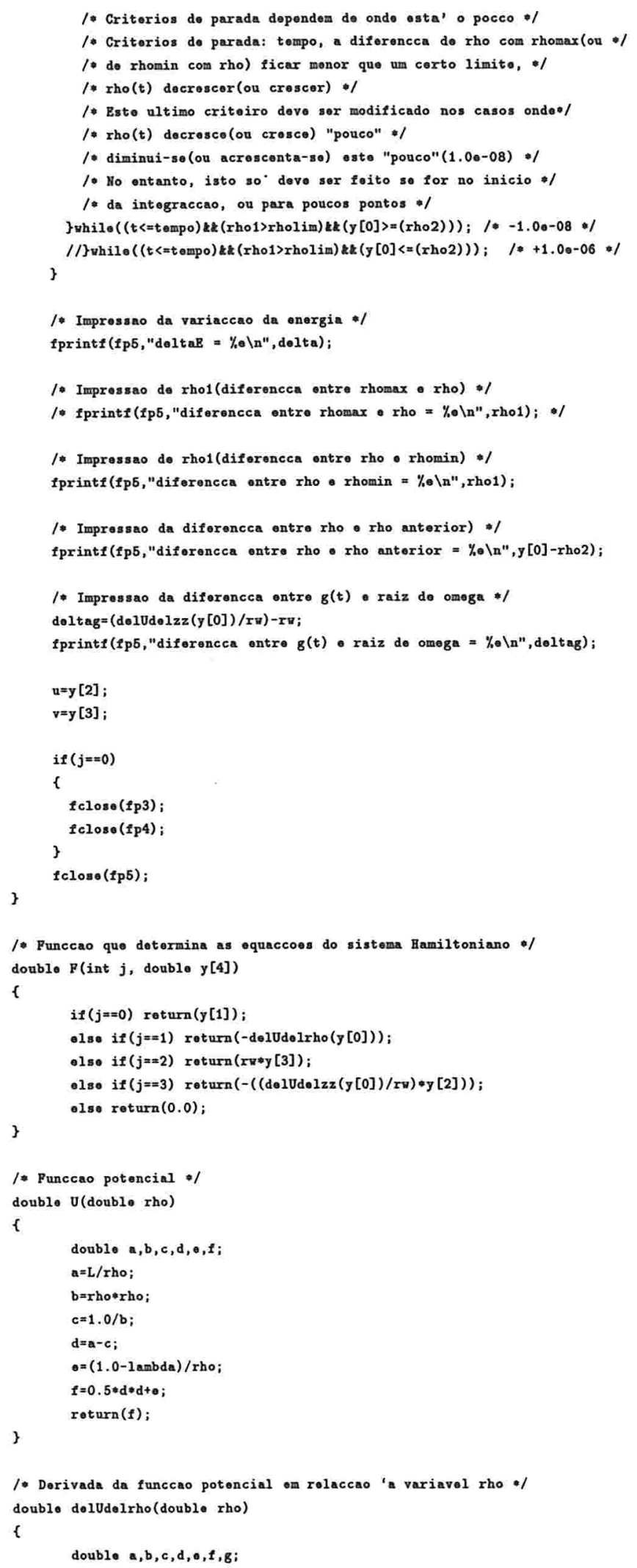




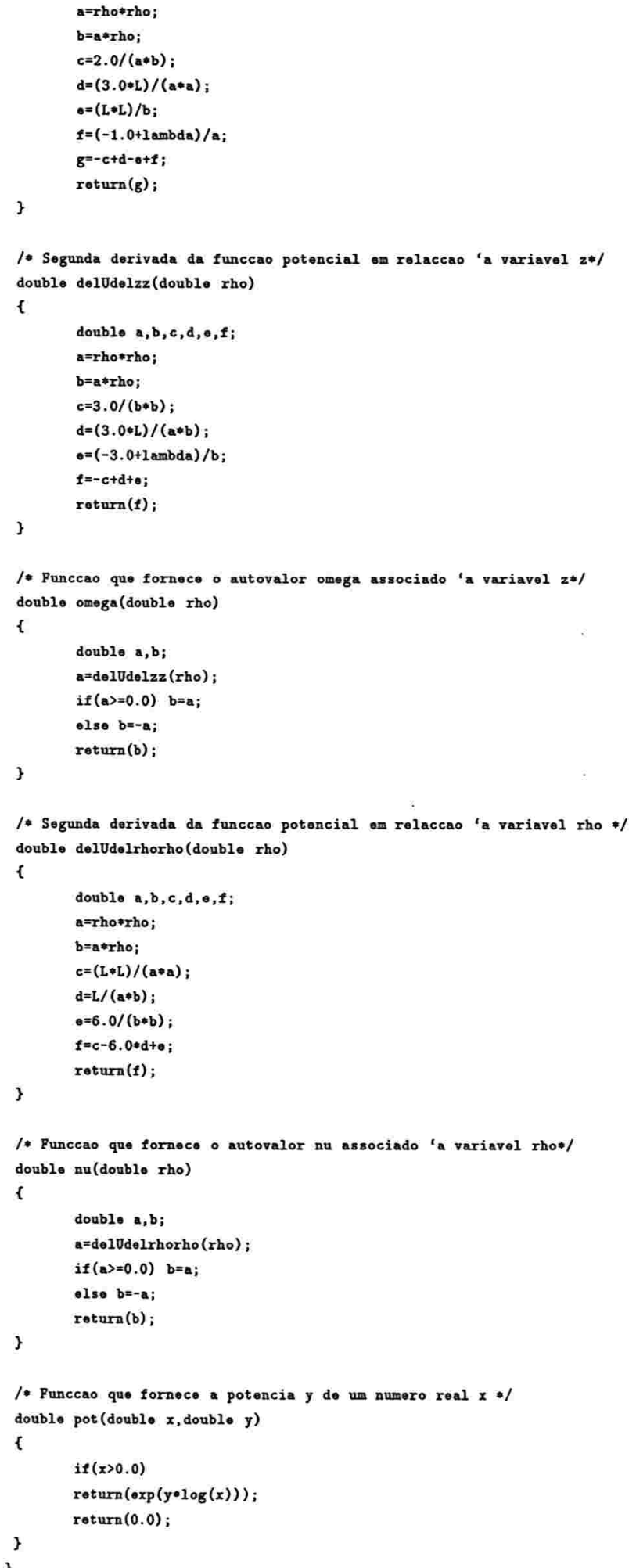

4. Programa para o cálculo da seção de Poincaré em $\left(z, p_{z}\right)$ para $\rho$ fixo 


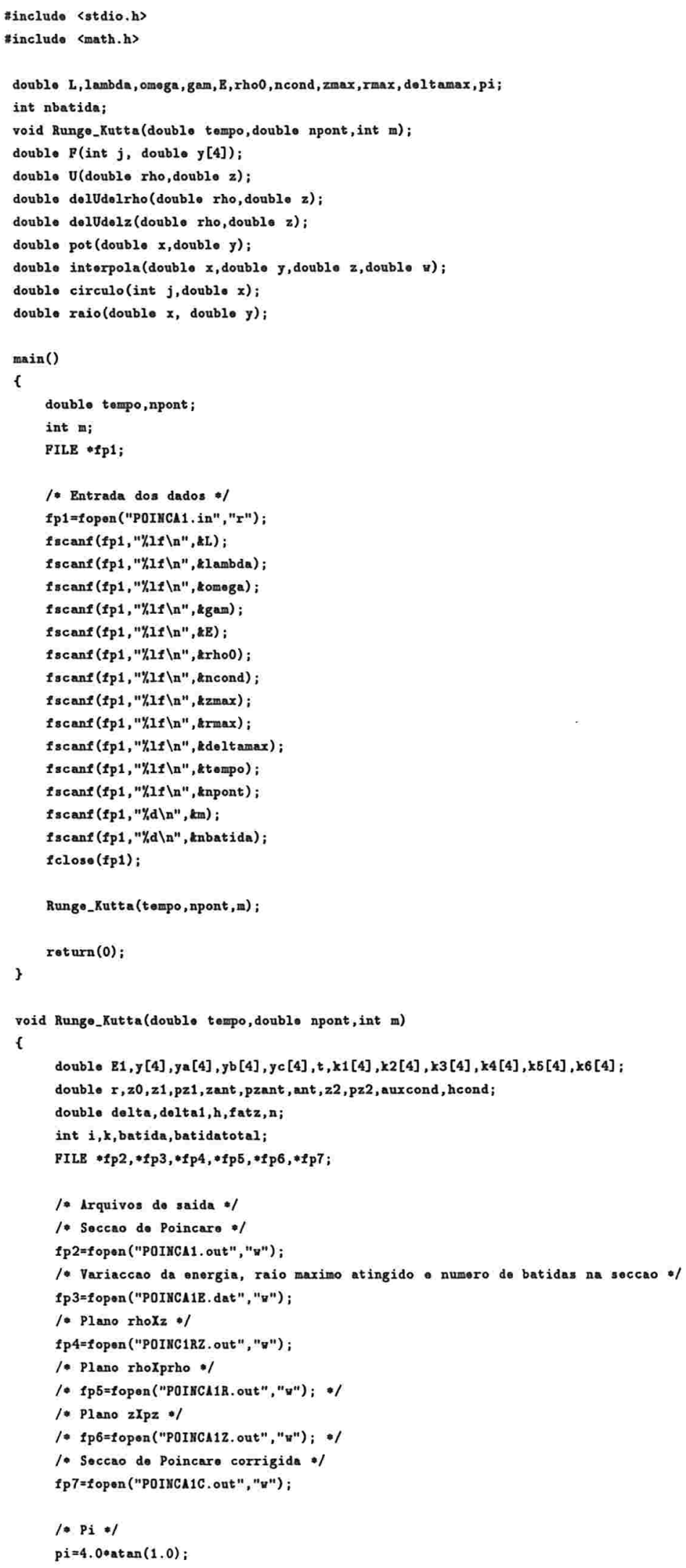




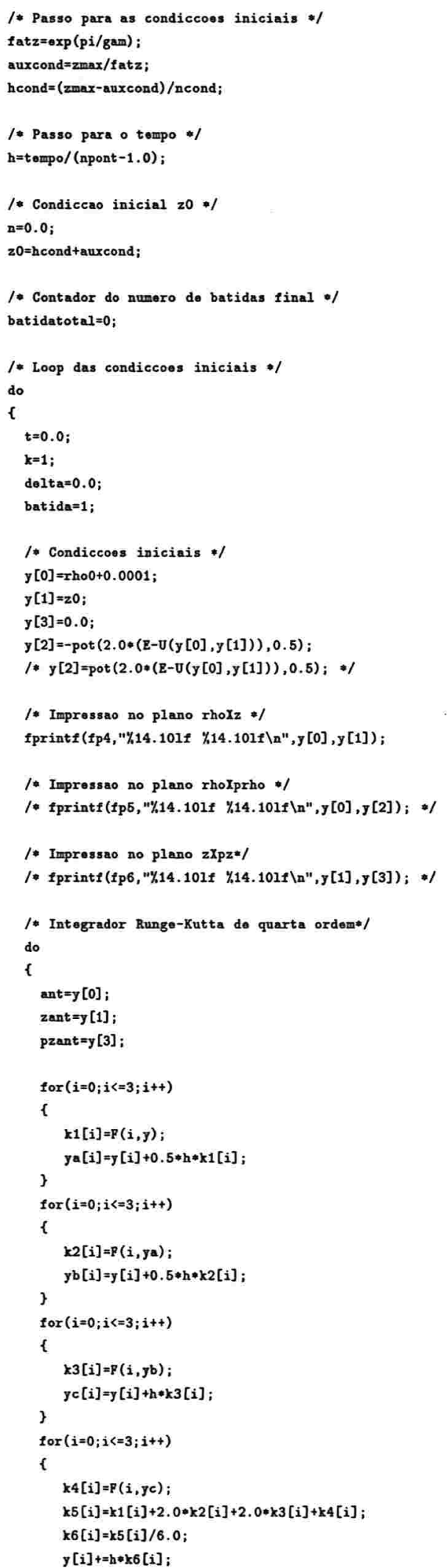


了

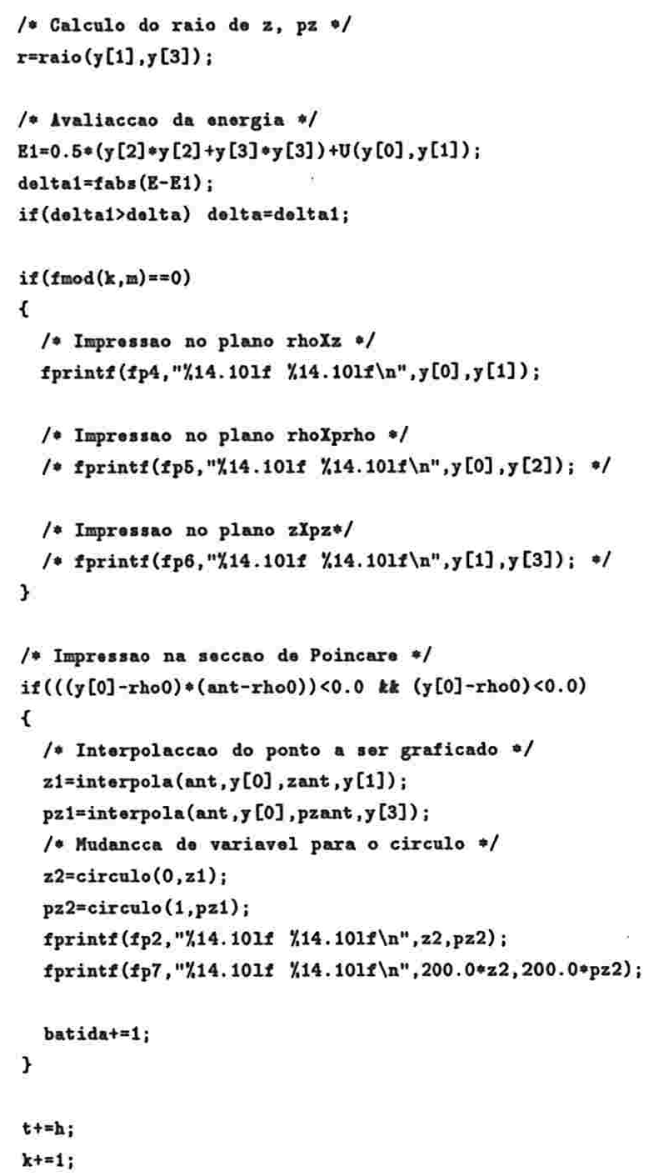


1. Punceao que dotermina as oquaccoes do sistema Hamiltoniano *f double $F$ (int $j$, double $y[4]$ )

l

it $(j==0)$ roturn $(y[2])$,

elso if $(j==1)$ return $(y[3])$;

else if $(j=2)$ retura $(-\mathrm{del} 10 \mathrm{del}$ rho $(\mathrm{y}[0], \mathrm{y}[1]))$;

else if $(j==3)$ return (-delodel $z(y[0], y[1]))$;

lse return $(0.0)$;

1* Funceao potencial *1

double $U$ (double rho, double $z$ )

r

double $a, b, c, d, e, f, g, h, i$;

a=rhoorho;

$\mathbf{b}=\mathbf{z} * \mathbf{z}$;

$c=a+b ;$

$d=\operatorname{pot}(c, 1.5)$;

$=(\mathrm{L} / \mathrm{rho})-(\mathrm{rho} / \mathrm{d})$.

$f=a / d$;

$g=\operatorname{pot}(c, 0.5)$;

$\mathrm{h}=1 \mathrm{ambda} / \mathrm{g}$;

$i=0.5 * 0 * 0+f-h$

$\operatorname{return}(i)$;

1. Derivada da funccao potencial em relaccao 'a variavel rho *I

double delUdelrho (double rho, double z)

double $a, b, c, d, o, f, g, h, i, j, k, 1$;

a=rhoorho;

$\mathbf{b}=\mathbf{z} * \mathbf{z}$;

$c=a+b$;

$d=$ pot $(c, 4.0)$

$\theta=($ rho* $(b-2.0 * a)) / d_{i}$

$f=$ pot $(c, 2.5)$;

$g=(3.0 * L *$ rho $) / f$;

$\mathrm{h}=(\mathrm{L} * \mathrm{~L}) /($ rho*a) ;

$i=(\operatorname{rho} *(2.0 * b-a)) / f$;

$j=p o t(c, 1.5)$;

$\mathbf{k}=($ lambda*rho $) / j$

$1=0+g-h+i+k$;

roturn(1);

1. Derivada da funceao potencial en relaccao 'a variavel z */ double deludelz (double rho, double z)

double $a, b, c, d, e, f, g, h, i, j ;$

$a=$ Tho+rho;

$\mathrm{b}=\mathbf{z} \mathbf{*} \mathbf{z}$;

$c=a+b$;

$d=$ pot $(c, 4.0)$;

$\theta=(a \cdot z) / d$

$f=\operatorname{pot}(c, 2.5)$;

$g=((L-a) * z) / f$

$h=\operatorname{pot}(c, 1.5)$;

$i=(1 \operatorname{ambda} * z) / \mathrm{h} ;$

$j=3.0 *(-\bullet+g)+i$;

$\operatorname{roturn}(j)$;

1. Punceao que fornece a potencia $y$ do um numera real $x *$ /

double pot (double $x$, double $y$ )

\&

if $(x>0.0)$

rotura $(\exp (y * \log (x)))$;

return $(0.0)$; 
s

1. Mudancea de variavel da elipso para o circulo ol doublo circulo (int $j$, doublo $x$ )

double $a, b$;

$a=p o t$ (omega , 0. 25) :

$b=1.0 / a$;

if $(j==0)$ return $(b * x)$;

olse if $(j==1)$ roturn $(a * x)$;

elso rotura $(0.0)$;

1: 8unccao que interpola o ponto a ser graficado */ doublo interpola (double $x$, doublo $y$, double $z$, double $y$ )

\{

double $a, b, c, d, e, f$;

$a=\mathbf{z}-\mathbf{z}$;

$b=y-r h o 0$;

$c=\mathbf{a} * b_{i}$

$\mathrm{d}=\mathrm{y}-\mathrm{x}$;

$\theta=c / d$;

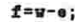

return(f);

1. Punccao que calcula o raio entro $\mathrm{z}$ - $\mathrm{pz}$ */ double raio(double $x$, double $y$ )

i

doublo $a, b, c, d$

$a=x+x ;$

$b=y * y$;

$c=a+b$;

$d=p o t(c, 0.5)$;

return(d);

5. Programa para a iteração da aplicação discreta em $\left(z, p_{z}\right)$

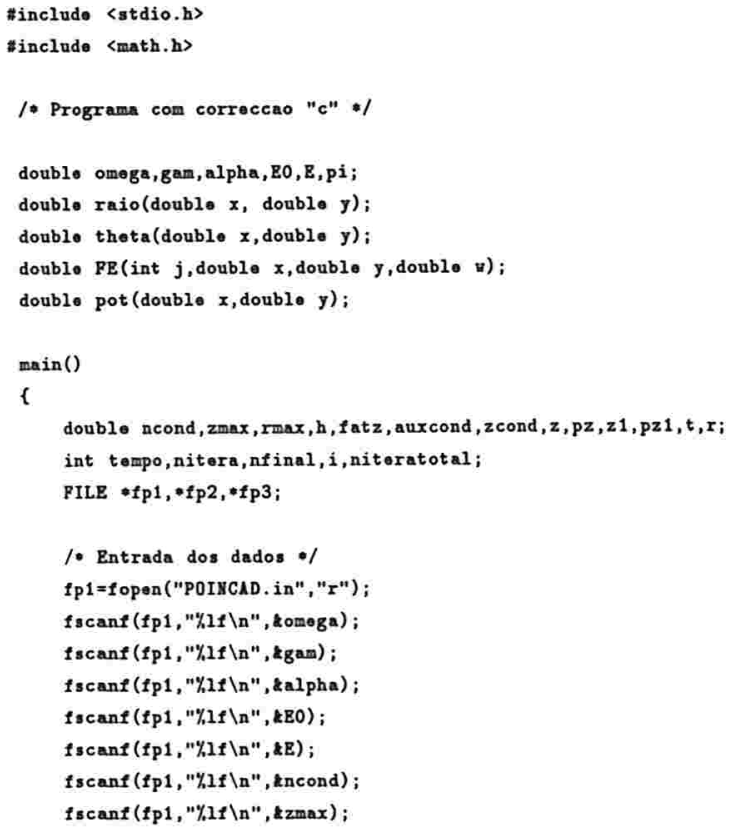


$f s c a n f(f p 1, " 1 /, 1 f \backslash n ", \operatorname{drmax})$;

f scanf ( $f \mathrm{p} 1, "$ " $\mathrm{d} \backslash \mathrm{n} "$, t tempo) ;

f scanf ( $p 1, " \% d \backslash n "$, znitera);

f scanf (fp1, "\%d\n", enfinal);

fclose $(f p 1)$.

1* Arquivos do saida *I

1. Iteradas da aplicaccao discreta */

fp2=fopen ("PoINCAD. out", " 8 "),

1. Numero de itoradas calculadas o raio maximo atingido */ fp3=fopen ("PoIrCAD. dat", " $"$ "),

$1 * \mathrm{Pi} * 1$

$p i=4.0 \cdot \operatorname{atan}(1.0)$

1. Passo para as condiccoos iniciais o/

fatz $=\exp (\mathrm{pi} / \mathrm{gam})$;

auxcond $=2 \max / \mathrm{fatz}$;

$\mathrm{h}=(\mathrm{zmax}-\mathrm{auxcond}) / \mathrm{ncond}$

1. Impressao da origem */

$z=0.0$;

$\mathrm{pz}=0.0$;

fprintf(fp2,"\%1f \%,1f $\backslash n ", z, p z)$;

1* Contador do numero de iteradas final */ niteratotal $=0$;

1. Loop da condiccao inicial $z$ \#

1. (eixo dos $z$ de htauxcond ato' zmax) */

for $(z \operatorname{cond}=\mathrm{h}$ taux $\operatorname{cond} ; \mathrm{zcond}<=\mathrm{zmax} ; \mathrm{zcond} \mathrm{t}=\mathrm{h}$ )

\{

1/ Condiccoss iniciais */

$i=1$;

$\mathrm{z} 1=\mathrm{zcond}$;

$\mathrm{pz} 1=0.0$

1* Loop do iteraccao da aplicaccao discreta FR */

do

- Calculo do theta */

$t=\operatorname{theta}(z 1, p z 1)$;

1* Calculo da iterada da aplicaccao discrota $\mathrm{FE}$ •/

$z=\mathrm{FE}(0, t, z 1, \mathrm{pz} 1)$;

$\mathrm{pz}=\mathrm{PE}(1, \mathrm{t}, \mathrm{z} 1, \mathrm{pz} 1)$;

/* Impressao da iterada da aplicaccao discreta PR */

fprintf(fp2,"\%,1f $\% / 1 f \backslash n ", z, p z)$;

1. Calculo do raio de $z, p z$ *)

$r=$ raio $(z, p z)$;

$\mathrm{z1}=\mathrm{z}$;

pz1=pz;

it+

\}vhile $((i<=t$ empo $) k z(x<=r \max ))$;

1* Impressao do numero de iteradas calculadas */

fprintf $(f \mathrm{p} 3$, "nitera $=\% d \backslash \mathrm{n} ", i-1)$;

1. Inpressao do raio maximo atingido - /

fprintf $(f \mathrm{p} 3$, "rmax $=\%(\mathrm{n} n ", r)$;

niteratotal $+=(i-1)$

1. Impressao do numero de iteradas calculadas e/

fprintf $(f \mathrm{p} 3$, "niteratotal $=\%$ d $\backslash n "$, niteratotal); 


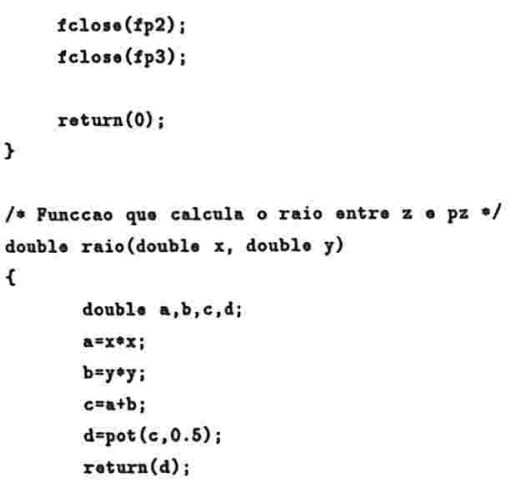


6. Programa para o cálculo da seção de Poincaré em $\left(z, p_{z}\right)$ para $\rho$ fixo, com o reescalamento para o anel

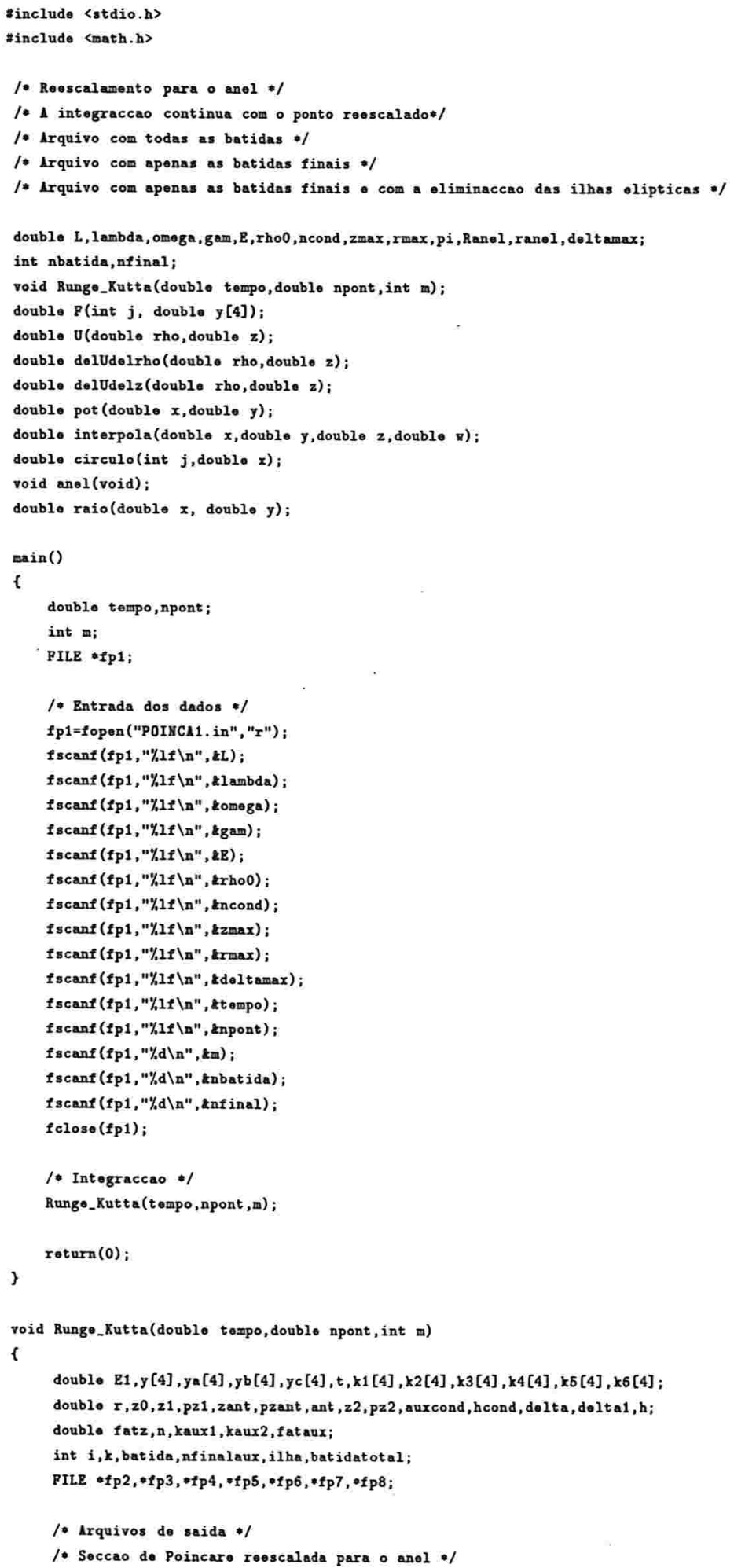




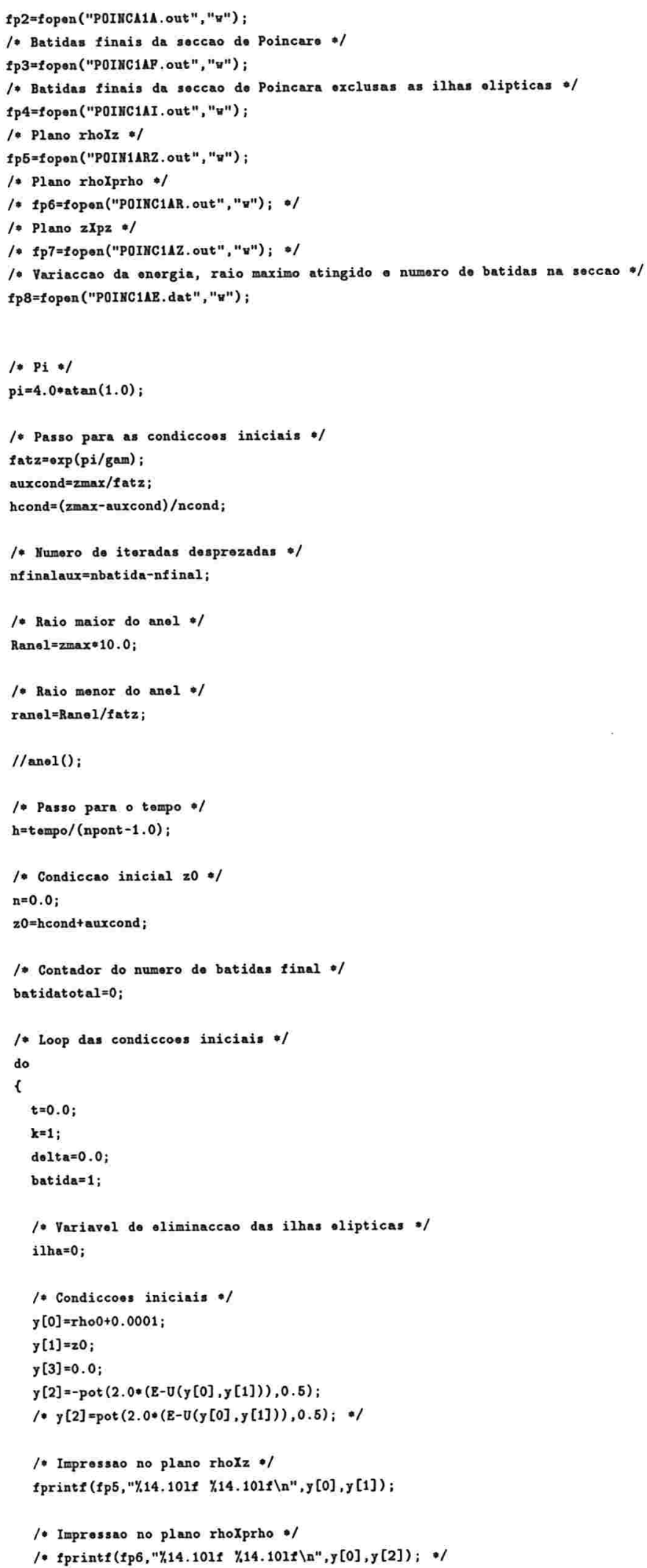




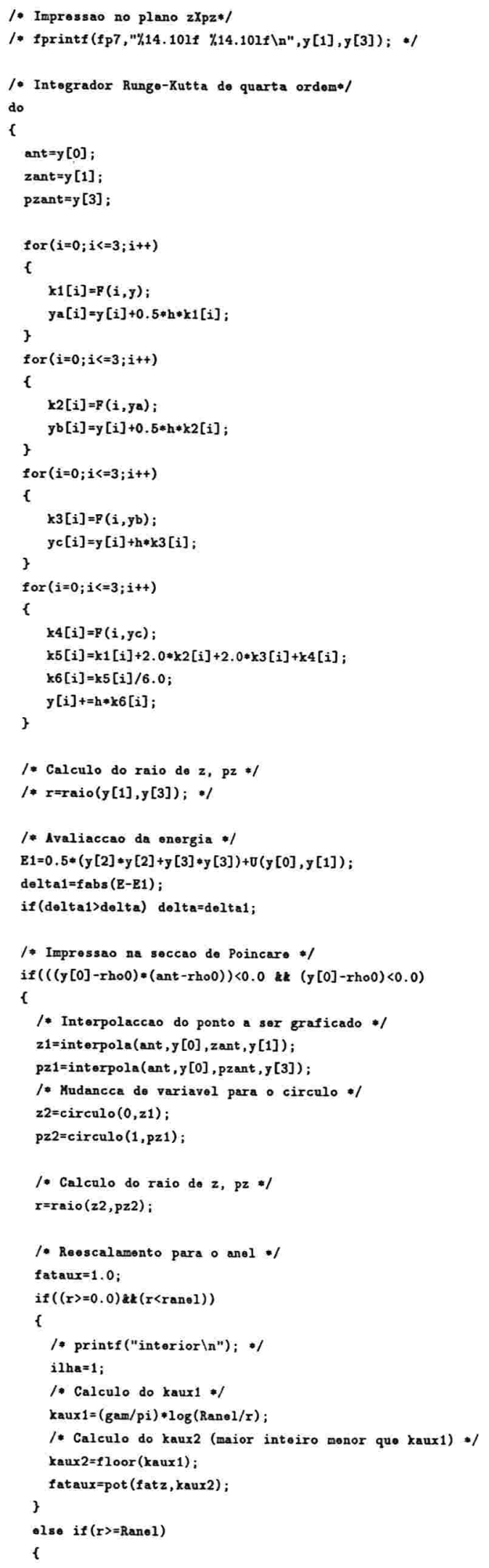




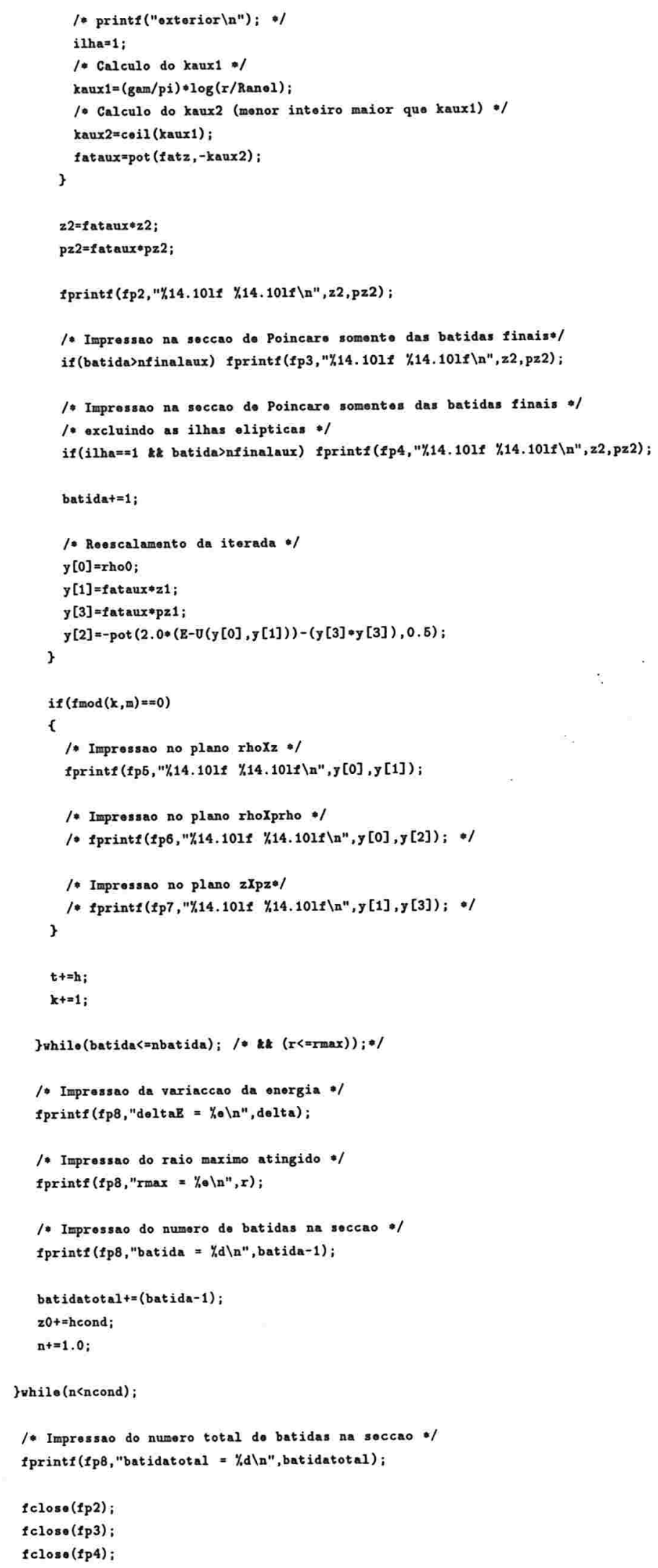


$f c l o s e(f p 5)$;

1* fclose(fp6); */

1* felose(fp 7$) ; * /$

fclose $\left(f_{p} 8\right)$;

* Punccao que determina as equaccoes do sistema Hamiltoniano double $F$ (int $j$, doublo $y[4]$ )

if $(j==0)$ return $(y[2])$;

else if $(j==1)$ return $(y[3])$;

else if $(j==2)$ roturn $(-d e l U d e l r h o(y[0], y[1]))$;

olse if $(j==3)$ return (-delvdelz $(y[0], y[1]))$;

else return $(0.0)$

1* Funccao potencial •/

double U(double rho, double $z$ )

double $a, b, c, d, e, f, g, h, i$;

$a=$ rhotrho;

$b=\mathbf{z}=\mathbf{z}$;

$c=a+b$;

$d=\operatorname{pot}(c, 1.5)$

$=(\mathrm{L} / \mathrm{rho})-(\mathrm{rho} / \mathrm{d})$;

$f=\mathrm{a} / \mathrm{d}$;

$g=\operatorname{pot}(c, 0.5)$;

$h=1 \operatorname{ambda} / g$;

$i=0.5 * \theta * \theta+f-h ;$

$\operatorname{return}(i)$;

1. Derivada da funccao potencial em relaccao 'a variavel rho */

double dolUdelrho (double rho, double $z$ )

double $a, b, c, d, e, f, g, h, i, j, k, 1$;

a=rho*rho;

$\mathbf{b}=\mathbf{z} * \mathbf{z}$;

$c=a+b$;

$d=$ pot $(c, 4.0)$;

$\theta=($ rho* $(b-2.0 * a)) / d$;

$t=$ pot $(c, 2.5)$;

$g=(3.0 *$ L*rho $) / f$;

$\mathrm{h}=(\mathrm{L} * \mathrm{~L}) /($ rho*a) $;$

$i=(\operatorname{rho*}(2.0 * b-a)) / f$;

$j=\operatorname{pot}(c, 1.5)$;

$k=($ lambda*rho $) / j$;

$1=\mathbf{e}+\mathrm{g}-\mathrm{h}+\mathrm{i}+\mathrm{k}$;

return(1);

* Derivada da funccao potencial em relaccao 'a variavel z */

double delvdelz (double rho, double z)

doublo $a, b, c, d, e, f, g, b, i, j$;

$a=$ rho*rho;

$b=z * z$;

$c=a+b$;

$d=\operatorname{pot}(c, 4.0)$;

$0=(a \cdot z) / d$;

$f=$ pot $(c, 2.5)$;

$g=((L-a) \cdot z) / f$

$h=p o t(c, 1.5)$;

$i=(1$ ambdat $z) / h$,

$j=3.0 \times(-e+g)+i$;

return $(j)$; 


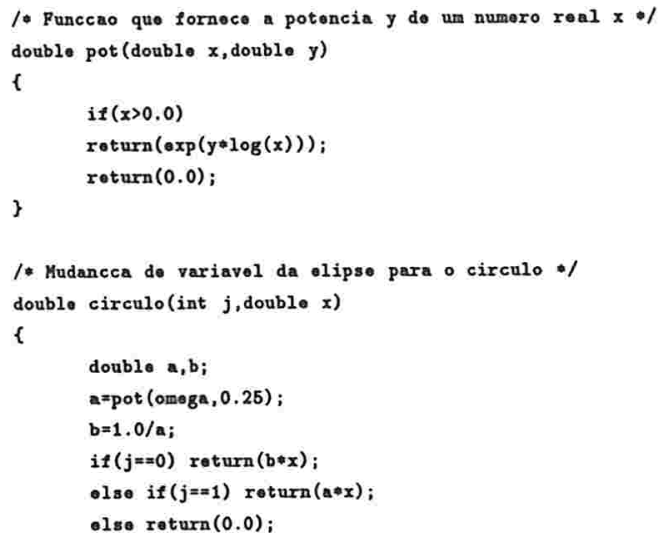




\section{Programa para iteração da aplicação discreta com o reescalamento para o anel}

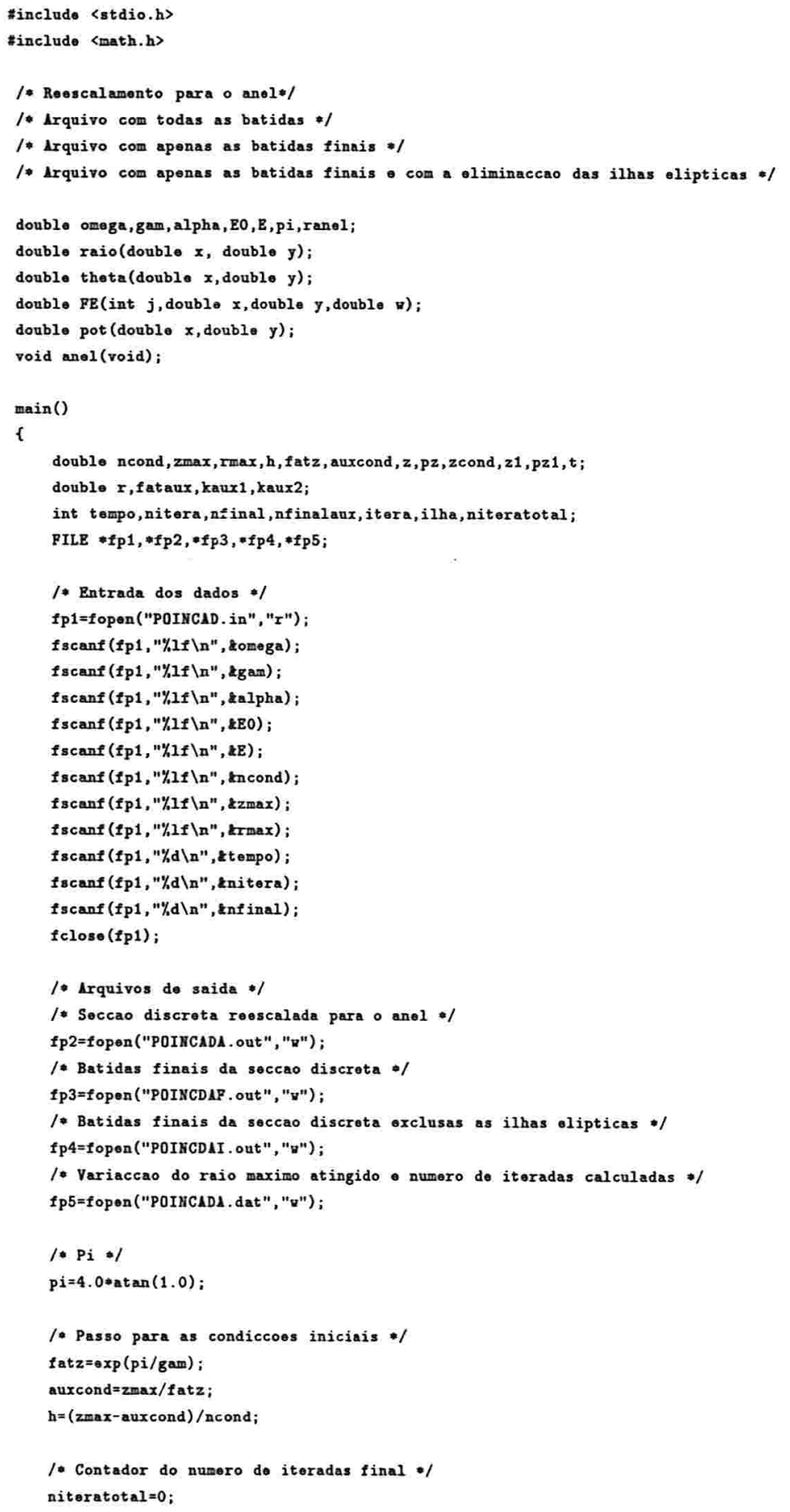




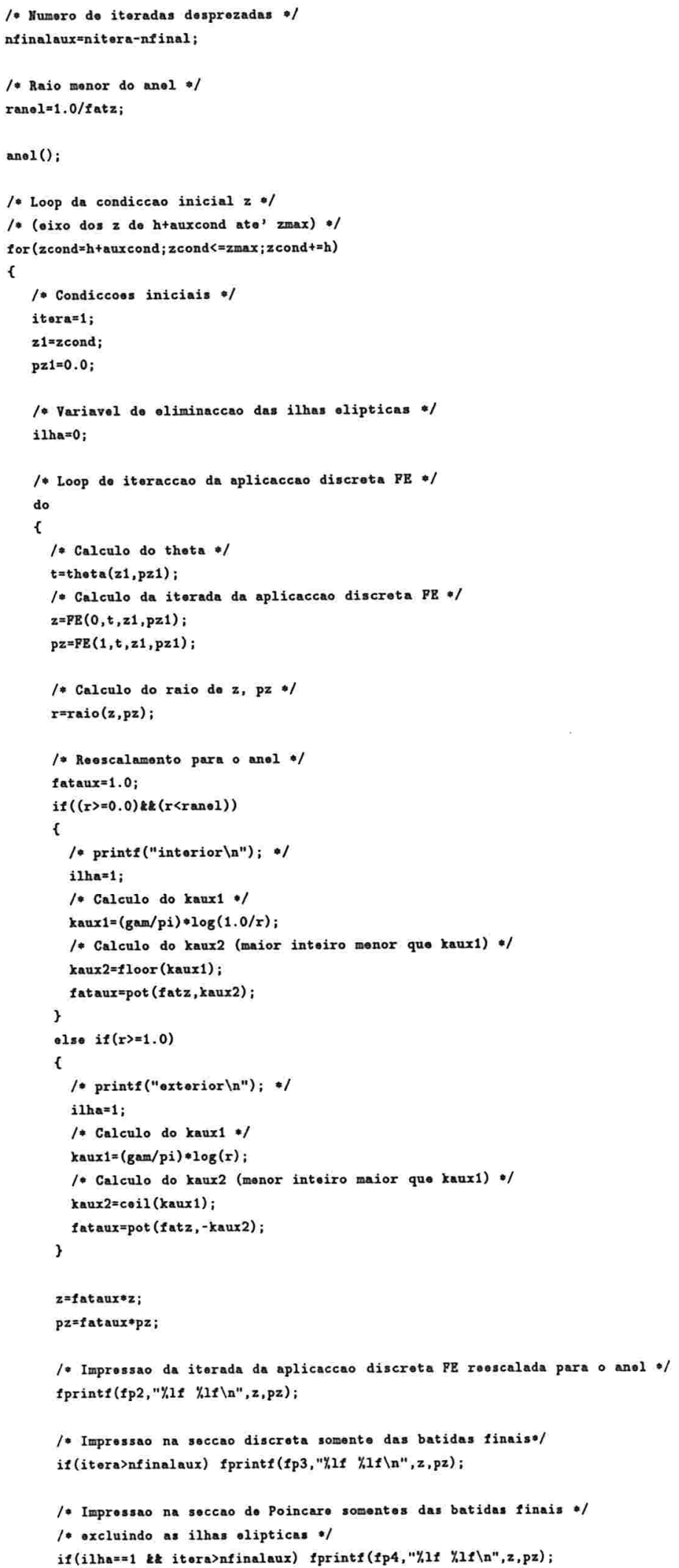




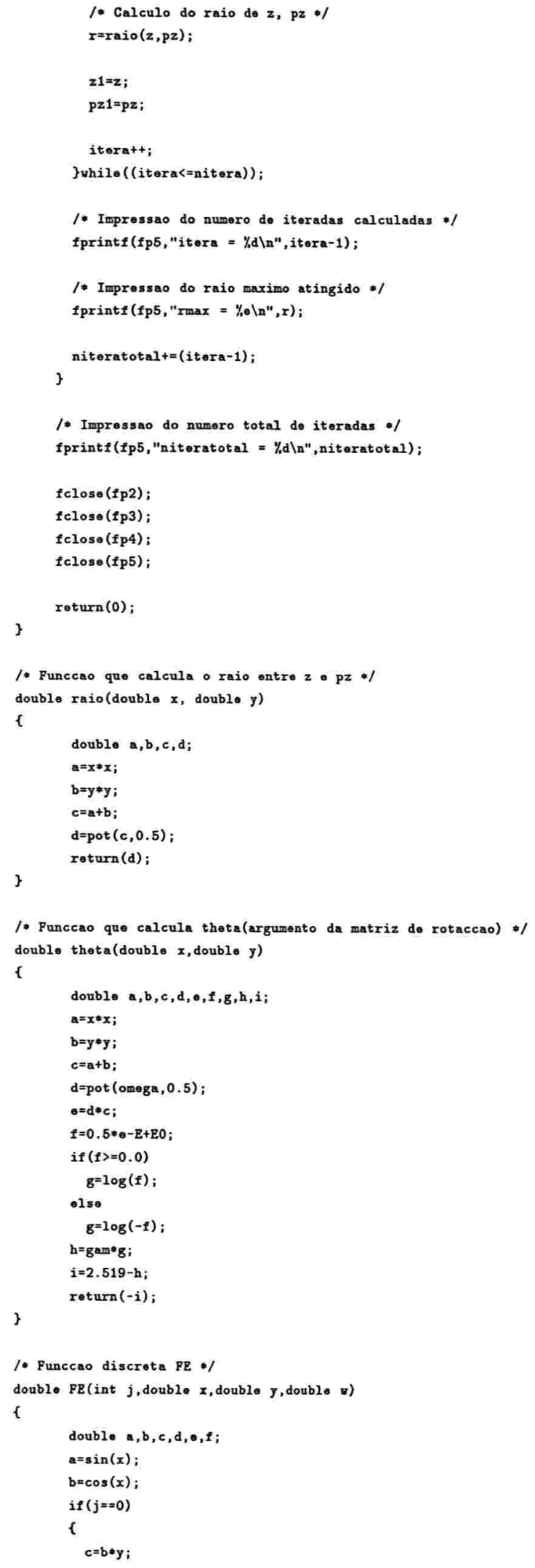




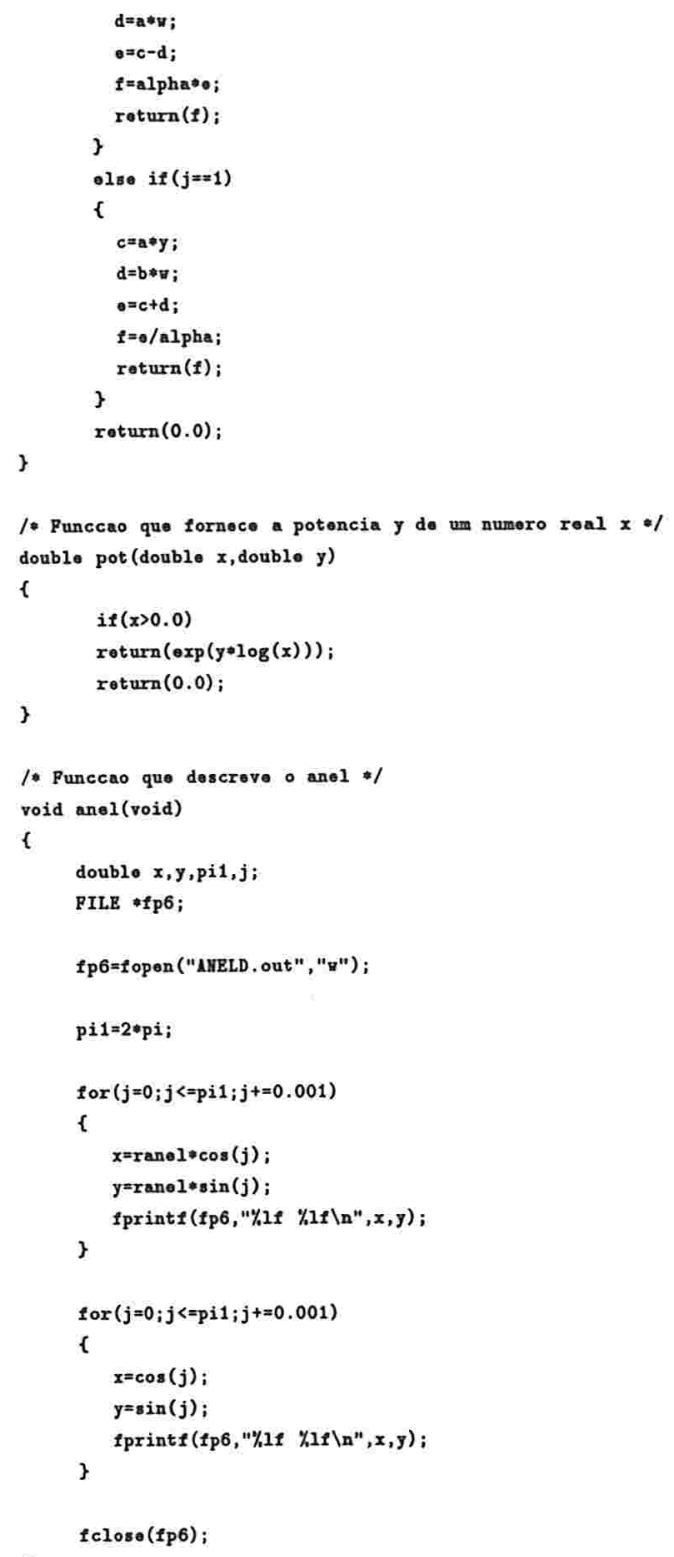

8. Programa para o cálculo da seção global de Poincaré em $\left(z, p_{z}\right)$ para $\rho$ fixo

\#include 〈stdio.h〉

"include 〈math.h〉

doubl. L, lambda, omega, gam, $E$, rhoo, ncond, zmax, rmax, del tamax, pi ;

void Runge_Kutta(double tempo,double npont, int m);

double $P$ (int $j$, double $y[4])$;

double U(double rho, double z);

double doludelrho(double rho, double z);

double deludelz (double rho, double z); 
double pot (double $x$, double $y$ );

double interpola (double $x$, donble $y$, double $z$, double $x$ );

double circulo(int $j$, double $x$ );

double raio(double $x$, double $y$ );

$\operatorname{main}()$

double tempo, npont;

int $m$;

PILE $* f$ p

1. Entrada dos dados */

fp1=fopen ("POIMCA1. in", "r");

fscanf $(f \mathrm{p} 1, " \%, 1 f \backslash \mathrm{n} ", k \mathrm{~L})$;

fscanf ( $f \mathrm{p} 1, " \%, 1 f \backslash \mathrm{n} ", \ell \operatorname{lambda})$

$f \operatorname{scanf}(p \mathrm{p} 1, n \% 1 f \backslash n "$, \&omega $)$;

fscanf ( $f p 1, " \%, f \backslash n ", \operatorname{lgam})$;

fscanf ( $f \mathrm{p} 1, " \%, 1 f \backslash \mathfrak{n} ", 2 E)$;

f scanf ( $\left.f_{P} 1, " \% / 1\right) \backslash n ", \&$ rhoo);

fscanf ( $f \mathrm{p} 1, " \% 1 f \backslash \mathrm{n} "$, \&ncond)

fscanf ( $f p 1, " \%, 1 f \backslash n ", \dot{z} z \max )$;

f scanf ( $f p 1, " \% 1 f \backslash n ", \& r \max )$;

fscanf ( $p 1, " 4,1 f \mid n ", k d e l$ tamax $)$.

fscanf ( $f \mathrm{p} 1$, " $/ 1 f \backslash \mathrm{n}$ ", et empo);

fscanf ( $f p 1, " \% 1 p \backslash n "$, enpont);

fscanf ( $f p 1, " \% d \backslash n ", k m)$;

fclose ( $f \mathrm{p} 1)$;

1. Integraccao $* 1$

Runge_Kutta (tempo, npont, m);

raturn $(0)$ :

void Runge_Rutta(double tempo, double npont, int $m$ )

double $\mathrm{E} 1, \mathrm{y}[4], \mathrm{ya}[4], \mathrm{yb}[4], \mathrm{yc}[4], t, \mathrm{k} 1[4], \mathrm{k} 2[4], \mathrm{k} 3[4], \mathrm{k} 4[4], \mathrm{k} 5[4], \mathrm{k} 6[4]$

doublo $r, z 0, z 1, p z 1, z a n t$, pzant, ant, $z 2, p z 2$, auxcond, hcond;

double delta, deltal, h, fatz, $n$;

int $i, k$, batida, batidatotal;

PILE $* \mathrm{p} 2, * f \mathrm{p} 3, \bullet f \mathrm{p} 4, \bullet \mathrm{p} 5, * f \mathrm{p} 6$

1* Arquivos de saida */

1. Seccao global de Poincare */

fp2=fopen ("POIHCA1G. out", "q");

1* Variaccao da energia, raio maximo atingido, numoro de batidas na seccao */

fP3=f open ("POINC1GE. dat", "q");

1. Plano rholz *1

fp4=fopen ( "POIN1GRZ. out", "q");

1. Plano rhoxprho *

1. fp5=fopen ("PoIrC1GR.out", "v"); */

1* P1ano zxpz *1

10 fp6=fopen ("PoINC1Gz.out", "u") ; */

1. Pi */

$p i=4.0 \cdot \operatorname{atan}(1.0)$;

1. Passo para as condiccoes iniciais •/

fat $z=\exp (\mathrm{pi} / \mathrm{gam})$;

auxcond $=z \max / \mathrm{fat} z$;

//hcond $=($ max - auxcond $) /$ incond;

hcond $=0.05$;

1. Passo para o tempo

$h=$ tempo/ (npont-1.0)

1* Condiccao inicial zo */

$\mathrm{n}=0.0$; 


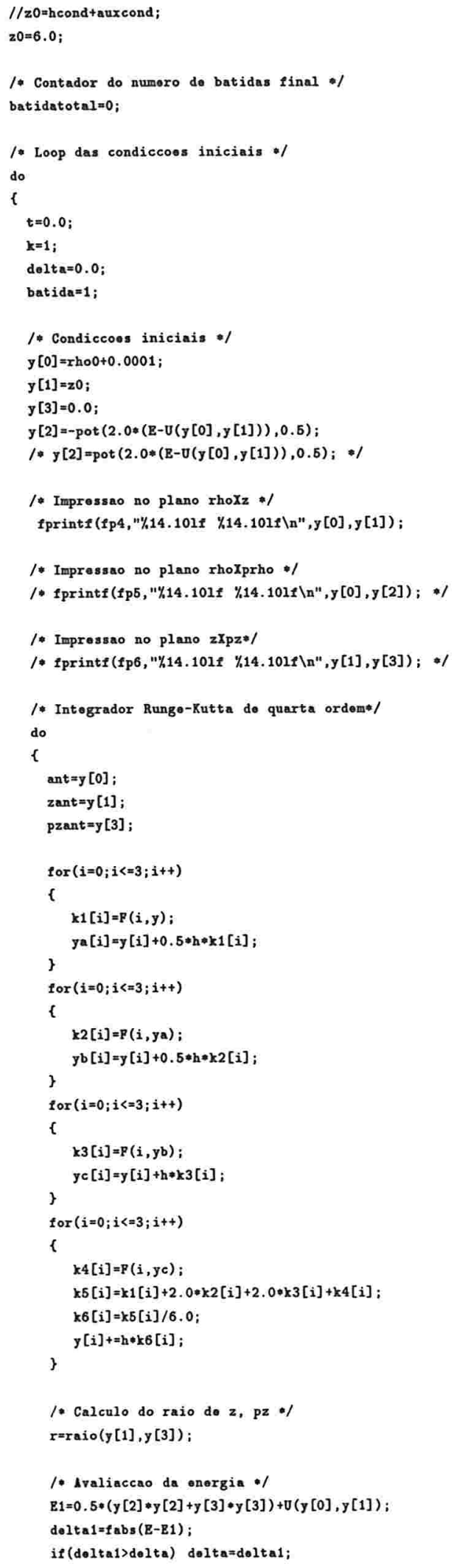




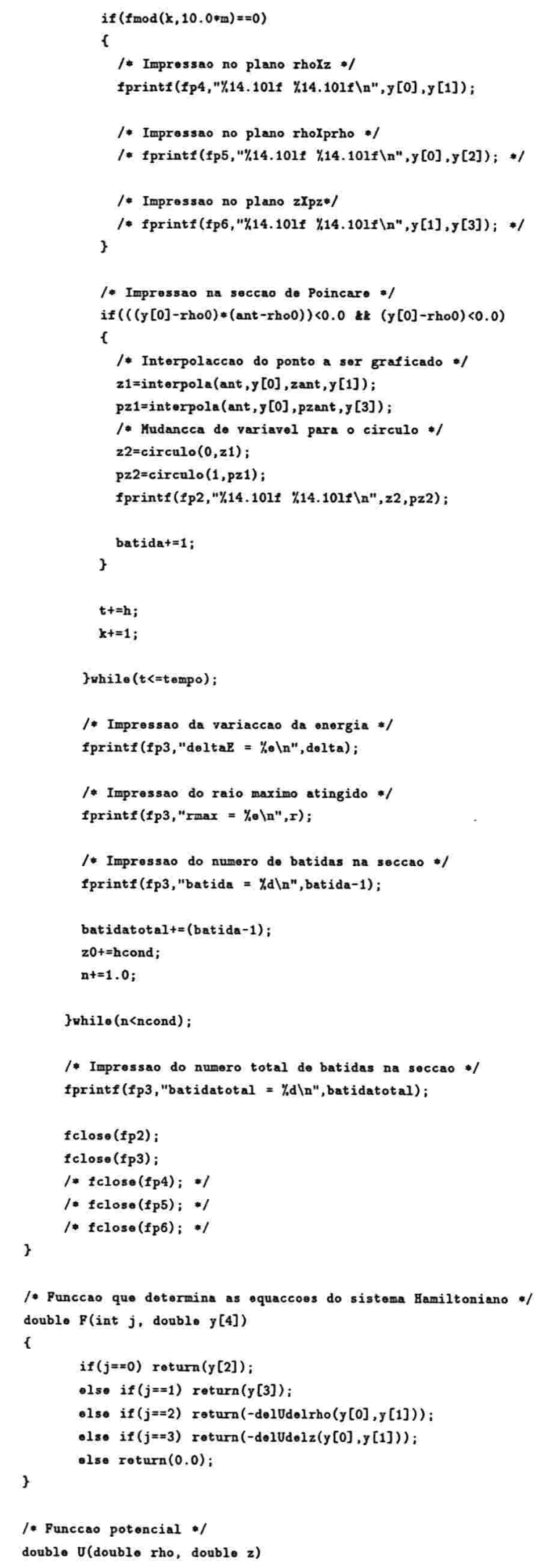




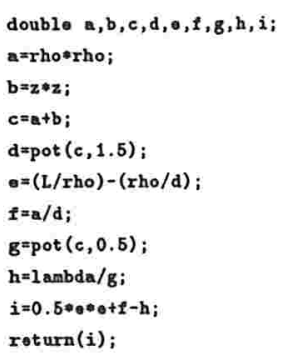




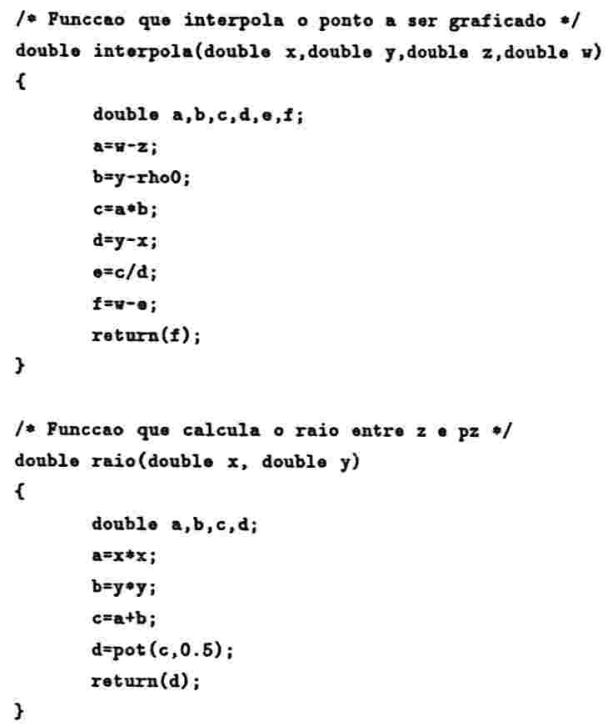

9. Programa para o cálculo da seção global de Poincaré em $\left(\rho, p_{\rho}\right)$ para $z$ fixo

\#include 〈stdio.h〉

\#include 〈math.h〉

double L, lambda, omega, E, rho0, prhoo, pi;

roid Runge_Kutta(double tempo, double npont, int $m$ );

double $P$ (int $j$, double $y[4])$;

double U(double rho, double $z$ );

doublo delvdolrho (double rho, double z);

double delUdelz (double rho, double z);

double pot (double $x$, double $y$ );

double interpola (double $x$, double $y$, double $z$, double $y$ ).

double circulo(int $j$, double $x$ );

double raio(double $x$, double $y$ );

$\operatorname{main}()$

f

double tempo, npont :

int $m_{i}$

PILE *fp1;

1. Entrada dos dados */

$f p 1=$ fopen ("POINCA1z.in", "r");

$f \mathrm{~g} \operatorname{cant}(f \mathrm{p} 1, " \mathrm{Y}, 1 f \backslash \mathrm{n} ", 2 \mathrm{~L})$.

$f \operatorname{scanf}(\mathrm{p} 1, " \% 1 f \backslash \mathrm{n} ", k$ ambda $)$;

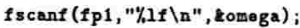

$f \operatorname{scanf}(f p 1, " \%, 1 f \backslash n ", \geq E)$;

f scant ( $f p 1, " \% 1\} \backslash n ", k r h o 0)$;

f scanf ( $f p 1, " \% 1 f \backslash n ", k p r h o 0)$;

iscant ( $f_{p} 1, " \% 1 f \backslash n ", k$ tempo)

f scanf ( $f p 1, " \% 1 f \backslash n "$, Enpont)

$f \operatorname{scanf}\left(f_{P} 1, " \% d \backslash n ", k m\right)$;

fclose(fp1);

1. Int ograccao */ 
Runge_Xutta (tempo,npont, m);

roturn (0);

\}

void Runge_Kutta(double tempo, double npont, int m)

‘

doublo $\mathrm{E} 1, \mathrm{y}[4], \mathrm{ya}[4], \mathrm{yb}[4], \mathrm{yc}[4], t, \mathrm{k} 1[4], \mathrm{k} 2[4], \mathrm{k} 3[4], \mathrm{k} 4[4], \mathrm{k} 5[4], \mathrm{k} 6[4]$

doublo $x$, rho1, prho1, rhoant, prhoant, ant, rho2, prho2, auxcond, heond;

double dolta,dolta1, $h, f a t z, n$;

int $i, k$, batida;

PILE $* f \mathrm{p} 2, * f \mathrm{p} 3, * f \mathrm{p} 4, * f \mathrm{p} 5, * f \mathrm{p} 6$;

1. Arquiros de saida *I

1. Seccao global de Poincare no plano rhoxprho cola z fixo

Ip2=fopon("POIMC1GZ . out", "w");

1. Variaccao da onorgia, raio maximo atingido numero do batidas na seccao of

fp3=fopen ("P0IIHGZE, dat", "q");

1\% Plano rhoxz o/

fp4=\{ open ("POI 1GZRZ, out", " $"$ ");

1. Plano zxrho o/

10 fp5=fopen("poIN1GZR. out", "q"); */

1* Plano zxpz *1

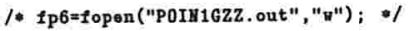

1* $\mathrm{Pi} * 1$

$p i=4.0 \neq \operatorname{atan}(1.0)$;

1. Passo para o tompo */

$h=$ tompo/(npont $-1,0)$;

$\mathrm{t}=0.0$;

$\mathrm{k}=1$;

delta $=0.0$;

batida $=1$;

1. Condiccoes iniciais o/

$\mathrm{y}[0]=\mathbf{r h o 0}$;

$y[1]=0.0001$;

$y[2]=$ prhoo;

$y[3]=-\operatorname{pot}(2.0 *(8-v(y[0], y[1])-(y[2] * y[2])), 0.5)$;

1* $y[3]=\operatorname{pot}(2.0 *(B-U(y[0], y[1])-(y[2] * y[2])), 0.5) ; * /$

1. Impressao no plano rholz */

fprintf(fp4,"\%,14.101f $\%, 14.101 f \backslash \mathrm{n} ", \mathrm{y}[0], \mathrm{y}[1])$;

1. Improssao no plano rhoxprho */

/. fprintf(fp5,"\%14.101f \%,14.101f $\backslash \mathrm{n} ", y[0], y[2]) ; * /$

1. Improssao no plano zxpz*/

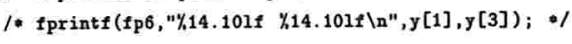

1. Integrador Runge-Kutta de quarta orden*/

do

ant $=y[1]$;

rhoant $=y[0]$

prhoant $=y[2]$;

for $(i=0 ; i<=3 ; i+4)$

f

$\mathrm{k} 1[\mathrm{i}]=\mathrm{P}(\mathrm{i}, \mathrm{y})$;

$y a[i]=y[i]+0.5 * h \circ k 1[i]$;

for $(i=0 ; i<=3 ; i++)$

\{

$\mathrm{k} 2[\mathrm{i}]=\mathbf{F}(\mathrm{i}, \mathrm{ya})$

$y b[i]=y[i]+0.5 * h * k 2[i]$; 


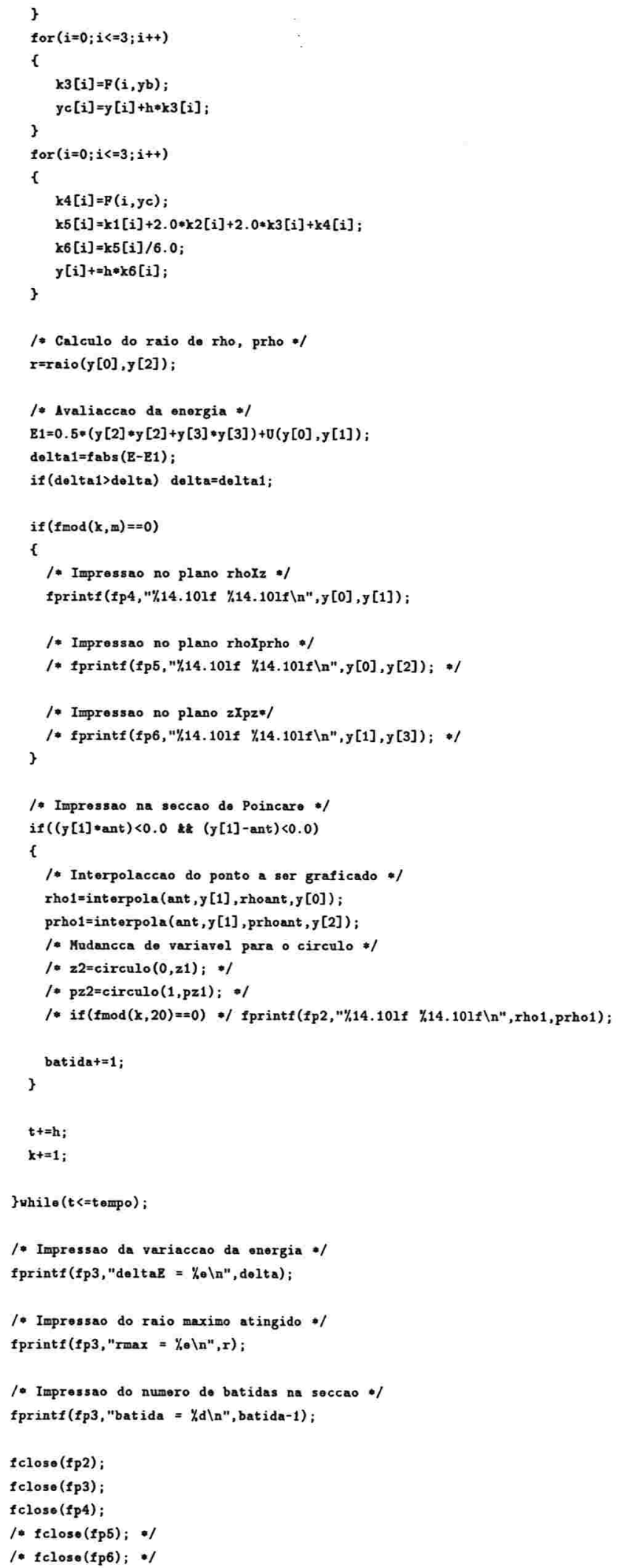




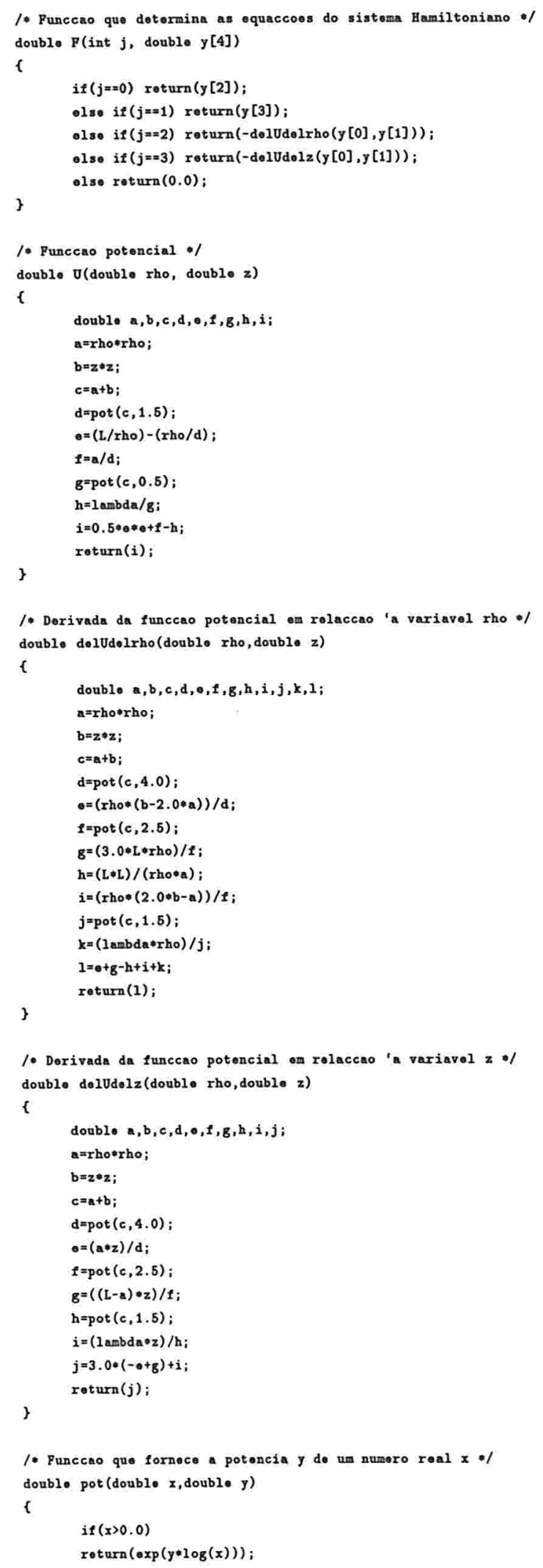


roturn $(0.0)$

1. Hudancca de variavel da olipse para o circulo */ double eirculo(int $j$, double $x$ )

భ

double $\mathbf{a}, \mathbf{b}$;

$a=p o t$ (omoga, 0. 25);

$b=1.0 / a$;

if $(j==0)$ return $(b * x)$.

else if $(j==1)$ return (a*x);

else return $(0.0)$.

1. Funccao que interpola o ponto a sor graficado -1

double interpola (double $x$, double $y$,double $z$, double $y$ )

\{

double $a, b, c, d, e$;

$a=z * y$;

$b=x=m ;$

$c=a-b$;

$\mathrm{d}=\mathrm{y}-\mathrm{x}$;

$e=c / d$;

retura(e);

1* Punccao que calcula o raio entre z \& pz */

double raio(double $x$, double $y$ )

f

double $a, b, c, d$

$a=x+$

$b=y * y$;

$c=a+b$;

$d=$ pot $(c, 0.5)$;

return (d); 


\section{Referências Bibliográficas}

Addas Zanata, S., Grotta Ragazzo, C. 2001. Critical number in scattering and escaping problems in classical mechanics. Phys. Rev. E 64, 046216.

Addas Zanata, S., Grotta Ragazzo, C. 2002. On the stability of some periodic orbitas of a new type for twist maps. Nonlinearity 15, 1385-1397.

Bastos de Figueiredo, J. C., Grotta Ragazzo, C., Malta, C. P. 1998. Two important numbers in the hénon-heiles dynamics. Physics Letters A 241, 35-40.

Burns, J., Showalter, M., Morfill, G. E. 1984. Planetary Rings. University of Arizona, Chapter The Ethereal Rings of Jupiter and Saturn, 200-272.

Burns, J. A., Showalter, M. R., Hamilton, D. P., Nicholson, P. D., De Pater, I., Ockert-Bell, M. E., Thomas, P. C. 1999. The formation of jupiter's faint rings. Science 284, 1146-1150.

Consolmagno, G. J. 1983. Lorentz forces on the dust in jupiter's ring. J. Geophys. Res. 88, A7, 5607-5612.

Dullin, H. R., Horányi, M., Howard, J. E. 2002. Generalizations of the störmer problem for dust grain orbits. Physica D 171, 178-195.

Grotta Ragazzo, C. 1994. Nonintegrability of some hamiltonian systems, scattering and analytic continuation. Comm. Math. Phys. 166, 255-277.

Grotta Ragazzo, C. 1997a. Irregular dynamics and homoclinic orbits to hamiltonian saddle centers. Comm. Pure App. Math. L, 105-147. 
Grotta Ragazzo, C. 1997b. On the stability of double homoclinic loops. Comm. Math. Phys. 184, 215-272.

Grotta Ragazzo, C. 1997c. Stability of homoclinics orbits and diffusion in phase space. Physics Letters A 230, 183-189.

Horányi, M., Cravens, T. E. 1996. The structure and dynamics of jupiter's rings. Nature 381, 293-295.

Howard, J. E., Dullin, H. R., Horányi, M. 2000. Stability of halo orbits. Phys. Rev. Lett. 84, 15, 3244-3247.

Howard, J. E., Horányi, M., Stewart, G. R. 1999. Global dynamics of charged dust particles in planetary magnetospheres. Phys. Rev. Lett. 83, 20, 39933996.

Krivov, A. V., Krüger, H., Grün, E., Thiessenhusen, K., Hamilton, D. P. 2002. A tenuous dust ring of jupiter formed by escaping ejecta from the galilean satellites. J. Geophys. R. 107, E1, 2.1-2.13.

LERMAN, L. M. 1991. Hamiltonian systems with loops of a separatrix of a saddlecenter. Sel. Math. Sov. 10, 3, 297-306.

Mendis, D. A., Houpis, H. L. F., Hill, J. R. 1982. The gravito-eletrodynamics of charged dust in planetary magnetospheres. J. Geophys. R. 87, A5, 3449-3455.

Mielke, A., Holmes, P., O'OReilly, O. 1992. Cascades of homoclinic orbits to, and chaor near, a hamiltonian saddle-center. J. Dyn. Diff. Eqns. 4, 1, 95-126.

Ockert-Bell, M. E., Burns, J. A., Daubar, I. J., Thomas, P. C., Veverka, J., Belton, M. J. S., KlaAsen, K. P. 1999. The strucuture of jupiter's ring system as revealed by the galileo imaging experiment. Icarus 138, 188-213.

Owen, T., Danielson, G. E., Cook, A. F., Hansen, C., Hall, V. C., DuXUBURY, T. 1979. Jupiters rings. Nature 281, 442-446.

Schaffer, L., Burns, J. A. 1994. Charged dust in planetary magnetospheres: Hamiltonian dynamics and numerical simulations for highly charged grains. J. Geophys. Res. 99, A9, 17211-17223. 
Smith, B. A., Soderblom, L. A., Johnson, T. V., Ingersoll, A. P., Collins, S. A., Shommaker, E. M., Hunt, G. E., Masursky, H., Carr, M. H., Davies, M. E., Cook, A. F., Boyce, J., Danielson, G. E., Owen, T., SAgan, C., Beebe, R. F., Veverka, J., Strom, R. G., McCauley, J. F., Morrison, D., Briggs, G. A., Suomi, V. E. 1979. Jupiter system through the eyes of voyager-1. Science 204, 951-972. 